\title{
Integral cohomology of the generalized Kummer fourfold
}

\author{
Simon Kapfer and Grégoire Menet
}

\begin{abstract}
We describe the integral cohomology of the generalized Kummer fourfold, giving an explicit basis, using Hilbert scheme cohomology and tools developed by Hassett and Tschinkel. Then, we apply our results to an irreducible holomorphic symplectic variety with singularities, obtained by a partial resolution of the generalized Kummer fourfold quotiented by a symplectic involution. We calculate the Beauville-Bogomolov form of this new variety, presenting the first example of such a form that is odd.
\end{abstract}

\section{Introduction}

In algebraic geometry, irreducible holomorphic symplectic (IHS) manifolds became important objects of study in recent years, after fundamental results by Beauville [Bea83] and Huybrechts [Huy99]. Among all the developments concerning this field, integral cohomology plays an inescapable role. This is primarily due to the Beauville-Bogomolov form, which is a non-degenerate symmetric integral and primitive bilinear pairing on the second cohomology group with integral coefficients. This form endows the second cohomology group with a lattice structure, establishing lattice theory as a fundamental tool omnipresent in all the last developments. As examples, we can cite works on classifications of automorphisms [Mon12, MTW18, BCS16] or the important survey of Markman [Mar11] with results on the Kähler cone and the monodromy. In a more modest manner, the fourth integral cohomology group is also quite useful. As examples, we can underline [BNS13, Theorem 1.2] providing formulas which apply for the classification of automorphism on IHS manifolds of $K 3^{[2]}$-type, particularly used in [BCS16]; furthermore, [Mar10, Theorem 1.10] provides a description of the monodromy group of the IHS manifolds of $K 3^{[n]}$-type; we can also cite [Men15], where the second author provides the Beauville-Bogomolov lattice of the Markushevich-Tikhomirov varieties constructed in [MT07]. Taking $X$ to be an IHS manifold of $K 3^{[2]}$-type, in all these papers a description of the torsion group $H^{4}(X, \mathbb{Z}) / \operatorname{Sym}^{2}\left(H^{2}(X, \mathbb{Z})\right)$ was essential.

Until now, no complete description of the integral cohomology of the generalized Kummer fourfold existed. In particular, the relation between the fourth cohomology group and the image of the symmetric power of the second cohomology group via the cup product was not known.

Received 16 November 2016, accepted in final form 27 May 2017.

2010 Mathematics Subject Classification 14J17, 14J35, 14F43.

Keywords: integral cohomology, generalized Kummer fourfolds, Nakajima operators, irreducible symplectic Vmanifolds, Beauville-Bogomolov form.

This journal is (C) Foundation Compositio Mathematica 2018. This article is distributed with Open Access under the terms of the Creative Commons Attribution Non-Commercial License, which permits non-commercial reuse, distribution, and reproduction in any medium, provided that the original work is properly cited. For commercial re-use, please contact the Foundation Compositio Mathematica.

GM is supported by Fapesp grant 2014/05733-9. SK was partially supported by a DAAD grant. 


\section{S. Kapfer And G. Menet}

For all reasons mentioned above, it appeared to us that this formed an interesting gap to fill.

Let $K_{2}(A)$ be the generalized Kummer fourfold over a torus $A$. There are three main theorems in this paper. Two of them describe the integral cohomology of the generalized Kummer fourfold:

- Theorem 6.31 provides an integral basis of $H^{4}\left(K_{2}(A), \mathbb{Z}\right)$ in terms of $\operatorname{Sym}^{2}\left(H^{2}\left(K_{2}(A), \mathbb{Z}\right)\right)$ and certain classes of Briançon subschemes with support on 3-torsion points, introduced in [HT13].

- Theorem 6.33 states that the pullback from the Hilbert scheme of points on the torus $\theta^{*}: H^{*}\left(A^{[3]}, \mathbb{Z}\right) \rightarrow H^{*}\left(K_{2}(A), \mathbb{Z}\right)$ is surjective except in degree 4 . Moreover, it provides an integral basis of $\operatorname{Im} \theta^{*}$ and shows that the kernel of $\theta^{*}$ is the ideal generated by $H^{1}\left(A^{[3]}, \mathbb{Z}\right)$.

The third theorem is related to irreducible symplectic $V$-manifolds; it can be seen as an application of Theorem 6.31 and a generalization of [Men15]. A V-manifold is a compact analytic complex space with at worst finite-quotient singularities. A V-manifold will be called symplectic if its non-singular locus is endowed with an everywhere non-degenerate holomorphic 2-form which extends to a resolution of singularities. A symplectic V-manifold will be called irreducible if it is complete and simply connected and if the holomorphic 2 -form is unique up to $\mathbb{C}^{*}$. Such varieties are good candidates to generalize the short list of known IHS manifolds, since some aspects of the theory were already generalized in [Nam01] and [Mat15], for instance the Beauville-Bogomolov form, the local Torelli theorem and the Fujiki formula.

In [Nam01], Namikawa proposes a definition of the Beauville-Bogomolov form for some singular irreducible symplectic varieties. He assumes that the singularities are only $\mathbb{Q}$-factorial with a singular locus of codimension at least 4 . Under these assumptions, he proves a local Torelli theorem. This result was completed by a generalization of the Fujiki formula by Matsushita in [Mat15] (see also Theorem 1.2.4 of [Men14] for a summarizing statement).

These results were further generalized by Kirschner for symplectic complex spaces in [Kir15]. The first concrete example of a Beauville-Bogomolov lattice for a singular irreducible symplectic variety has appeared in [Men15, Theorem 2.5]. The variety studied in [Men15] is a partial resolution of an irreducible symplectic manifold of $K 3^{[2]}$-type quotiented by a symplectic involution. The objective of this paper is to provide a new example of a Beauville-Bogomolov lattice, by replacing the manifold of $K 3^{[2]}$-type by a fourfold of Kummer type. Knowing the integral basis of the cohomology group of the generalized Kummer provided by Theorem 6.31 renders this calculation possible. Moreover, the calculation will be much simpler than in [Men15] because of the general techniques for calculating integral cohomology of quotients developed in [Men14] and the new technique using monodromy developed in Lemma 8.17. The other techniques developed in [Men15] are also contained in [Men14], so to simplify the reading, we will only cite [Men15] in the rest of the article.

Concretely, let $X$ be an irreducible symplectic fourfold of Kummer type and $\iota$ a symplectic involution on $X$. Theorem 7.5 establishes that the fixed locus of $\iota$ is the union of 36 points and a K3 surface $Z_{0}$. Then, the singular locus of $K:=X / \iota$ is the union of a K3 surface and 36 points. The singular locus is not of codimension 4 . We will lift to a partial resolution of singularities $K^{\prime}$ of $K$ obtained by blowing up the image of $Z_{0}$. By [Fuj83, Section 2.3 and Lemma 1.2], the variety $K^{\prime}$ is an irreducible symplectic V-manifold which has singular locus of codimension 4.

TheOrem 1.1. Let $X$ be an irreducible symplectic fourfold of Kummer type and $\iota$ a symplectic involution on $X$. Let $Z_{0}$ be the K3 surface which is in the fixed locus of $\iota$. We set $K=X / \iota$ and denote by $K^{\prime}$ the partial resolution of singularities of $K$ obtained by blowing up the image 


\section{INTEGRAL COHOMOLOGY OF THE GENERALIZED KUMMER FOURFOLD}

of $Z_{0}$. Then the Beauville-Bogomolov lattice $H^{2}\left(K^{\prime}, \mathbb{Z}\right)$ is isomorphic to $U(3)^{3} \oplus\left(\begin{array}{l}-5-4 \\ -4-5\end{array}\right)$, and the Fujiki constant $c_{K^{\prime}}$ is equal to 8.

We remark that this is the first example of a Beauville-Bogomolov form which is not even.

The paper is organized as follows. In Section 2, we describe the odd integral cohomology of $A^{[2]}$, the Hilbert scheme of two points on a surface $A$ with torsion-free cohomology. Then, after recalling some notions on Nakajima operators in Section 3, we are able to provide an integral basis of the Hilbert scheme of two points on an abelian surface in term of Nakajima operators (Proposition 4.6). Section 5 studies the integral cohomology of generalized Kummer varieties in any dimension. In Section 6.4, we use all these preliminary results and the monodromy technique developed in [HT13] to find an integral basis of the cohomology of the generalized Kummer fourfold $K_{2}(A)$. As a consequence, in Section 7, we are able to complete the classification of symplectic involutions on $K_{2}(A)$ as a corollary of the lattice classification by Mongardi, Tari and Wandel in [MTW18]. Finally, Section 8 is dedicated to the proof of Theorem 1.1.

\section{Odd cohomology of $A^{[2]}$}

Let $A$ be a smooth compact surface with torsion-free cohomology and $A^{[2]}$ the Hilbert scheme of two points. It can be constructed as follows: Consider the direct product $A \times A$. Let

$$
b: \mathrm{Bl}_{\Delta}(A \times A) \rightarrow A \times A
$$

be the blow-up along the diagonal $\Delta \cong A$ with exceptional divisor $E$. Let $j: E \rightarrow \mathrm{Bl}_{\Delta}(A \times A)$ be the embedding. The action of $\mathfrak{S}_{2}$ on $A \times A$ lifts to an action on $\mathrm{Bl}_{\Delta}(A \times A)$. We have the push-forward $j_{*}: H^{*}(E, \mathbb{Z}) \rightarrow H^{*}\left(\mathrm{Bl}_{\Delta}(A \times A), \mathbb{Z}\right)$.

The quotient by the action of $\mathfrak{S}_{2}$ is $\pi: \mathrm{Bl}_{\Delta}(A \times A) \rightarrow A^{[2]}$. Now, $A^{[2]}$ is a compact complex manifold with torsion-free cohomology [Tot16, Theorem 2.2]. In this section, we want to prove the following proposition.

Proposition 2.1. Let $A$ be a smooth compact surface with torsion-free cohomology. Then

(i) $H^{3}\left(A^{[2]}, \mathbb{Z}\right)=\pi_{*}\left(b^{*}\left(H^{3}(A \times A, \mathbb{Z})\right)\right) \oplus \frac{1}{2} \pi_{*} j_{*} b_{\mid E}^{*}\left(H^{1}(\Delta, \mathbb{Z})\right)$;

(ii) $H^{5}\left(A^{[2]}, \mathbb{Z}\right)=\pi_{*}\left(b^{*}\left(H^{5}(A \times A, \mathbb{Z})\right)\right) \oplus \pi_{*} j_{*} b_{\mid E}^{*}\left(H^{3}(\Delta, \mathbb{Z})\right)$.

We adopt the following notation.

Notation 2.2. We denote the generators of $H^{1}(A, \mathbb{Z})$ by $a_{i}$ and their respective duals by $a_{i}^{*} \in$ $H^{3}(A, \mathbb{Z})$. We denote the generator of the top cohomology $H^{4}(A, \mathbb{Z})$ by $x$. A basis of $H^{2}(A, \mathbb{Z})$ will be denoted by $\left(b_{i}\right)$.

The rest of this section is dedicated to the proof of Proposition 2.1, using techniques developed in [Men14]; for another approach, see [Tot16]. The proof is organized as follows. First, we recall some notions on integral cohomology endowed with the action of an involution in Section 2.1. Then, Section 2.2 is devoted to calculating the torsion of $H^{3}\left(A^{[2]} \backslash E, \mathbb{Z}\right)$ (Lemma 2.10) using equivariant cohomology techniques. Then, this knowledge allow us to deduce part (i) using the exact sequence (2.5) of Section 2.3 and part (ii) using the unimodularity of the lattice $H^{3}\left(\mathrm{Bl}_{\Delta}(A \times\right.$ $A), \mathbb{Z}) \oplus H^{5}\left(\mathrm{Bl}_{\Delta}(A \times A), \mathbb{Z}\right)$.

\subsection{Integral cohomology under the action of an involution}

The main references for this subsection are [Men14] and [BNS13]. 


\section{S. Kapfer And G. Menet}

Let $G=\langle\iota\rangle$ be the group generated by an involution $\iota$ on a complex manifold $X$. As in [BNS13, Section 5], let $\mathcal{O}_{K}$ be the ring $\mathbb{Z}$ with the following $G$-module structure: $\iota \cdot y=-y$ for $y \in \mathcal{O}_{K}$. For $a \in \mathbb{Z}$, we denote by $\left(\mathcal{O}_{K}, a\right)$ the module $\mathbb{Z} \oplus \mathbb{Z}$ whose $G$-module structure is defined by $\iota \cdot(y, k)=(-y+k a, k)$. We denote the $\mathbb{F}_{2}[G]$-module $\left(\mathcal{O}_{K}, a\right) \otimes \mathbb{F}_{2}$ by $N_{2}$. We recall [Men14, Definition-Proposition 2.2.2].

Definition-Proposition 2.3. Assume that $H^{*}(X, \mathbb{Z})$ is torsion free. Then for all $0 \leqslant k \leqslant$ $2 \operatorname{dim} X$, we have an isomorphism of $\mathbb{Z}[G]$-modules

$$
H^{k}(X, \mathbb{Z}) \simeq \bigoplus_{i=1}^{r}\left(\mathcal{O}_{K}, a_{i}\right) \oplus \mathcal{O}_{K}^{\oplus s} \oplus \mathbb{Z}^{\oplus t}
$$

for some odd numbers $a_{i}$ and $(r, s, t) \in \mathbb{N}^{3}$. We get the following isomorphism of $\mathbb{F}_{2}[G]$-modules:

$$
H^{k}\left(X, \mathbb{F}_{2}\right) \simeq N_{2}^{\oplus r} \oplus \mathbb{F}_{2}^{\oplus(s+t)} .
$$

We set $l_{2}^{k}(X):=r, l_{1,-}^{k}(X):=s, l_{1,+}^{k}(X):=t, \mathcal{N}_{2}:=N_{2}^{\oplus r}$ and $\mathcal{N}_{1}:=\mathbb{F}_{2}^{\oplus s+t}$.

Remark 2.4. These invariants are uniquely determined by $G, X$ and $k$.

Proposition 2.5 ([Men14, Section 2.2]). Let $X$ be a compact complex manifold of dimension $n$ and $\iota$ an involution. Assume that $H^{*}(X, \mathbb{Z})$ is torsion free.

(i) $\operatorname{rk} H^{k}(X, \mathbb{Z})^{\iota}=l_{2}^{k}(X)+l_{1,+}^{k}(X)$.

(ii) Set $\sigma:=\mathrm{id}+\iota^{*}$ and $S_{\iota}^{k}:=\operatorname{Ker} \sigma \cap H^{k}(X, \mathbb{Z})$. We have $H^{k}(X, \mathbb{Z})^{\iota} \cap S_{\iota}^{k}=0$ and

$$
\frac{H^{k}(X, \mathbb{Z})}{H^{k}(X, \mathbb{Z})^{\iota} \oplus S_{\iota}^{k}}=\left(\frac{\mathbb{Z}}{2 \mathbb{Z}}\right)^{\oplus l_{2}^{k}(X)} .
$$

Remark 2.6. Note that the elements of $\left(\mathcal{O}_{K}, a_{i}\right)^{\iota}$ are written $y+\iota^{*}(y)$ with $y \in\left(\mathcal{O}_{K}, a_{i}\right)$.

Let $\pi: X \rightarrow X / G$ be the quotient map. We denote the pullback and the push-forward along $\pi$ by $\pi^{*}$ and $\pi_{*}$, respectively. We recall that

$$
\pi_{*} \circ \pi^{*}=2 \mathrm{id} \text { and } \pi^{*} \circ \pi_{*}=\mathrm{id}+\iota^{*} .
$$

We also recall the commutativity of $\pi_{*}$ with the cup product.

Proposition 2.7 ([Men14, Lemma 3.3.7]). Let $X$ be a compact complex manifold of dimension $n$ and $\iota$ an involution. Assume that $H^{*}(X, \mathbb{Z})$ is torsion free. Let $0 \leqslant k \leqslant 2 n$, let $m$ be an integer such that $k m \leqslant 2 n$, and let $\left(x_{i}\right)_{1 \leqslant i \leqslant m}$ be elements of $H^{k}(X, \mathbb{Z})^{\iota}$. Then

$$
\pi_{*}\left(x_{1}\right) \cdot \ldots \cdot \pi_{*}\left(x_{m}\right)=2^{m-1} \pi_{*}\left(x_{1} \cdot \ldots \cdot x_{m}\right) .
$$

\subsection{Preliminary lemmas}

We set $V=\mathrm{Bl}_{\Delta}(A \times A) \backslash E$ and $U=V / \mathfrak{S}_{2}=A^{[2]} \backslash E$, where $\mathfrak{S}_{2}=\left\langle\sigma_{2}\right\rangle$.

Lemma 2.8. We have $H^{k}(A \times A, \mathbb{Z})=H^{k}(V, \mathbb{Z})$ for all $k \leqslant 3$.

Proof. We have $V \cong A \times A \backslash \Delta$, so we get the following natural exact sequence:

$$
\cdots \longrightarrow H^{k}(A \times A, V, \mathbb{Z}) \longrightarrow H^{k}(A \times A, \mathbb{Z}) \longrightarrow H^{k}(V, \mathbb{Z}) \longrightarrow \cdots .
$$

Moreover, by the Thom isomorphism, $H^{k}(A \times A, V, \mathbb{Z})=H^{k-4}(\Delta, \mathbb{Z})=H^{k-4}(A, \mathbb{Z})$. Hence, $H^{k}(A \times A, V, \mathbb{Z})=0$ for all $k \leqslant 3$. Consequently, $H^{k}(A \times A, \mathbb{Z})=H^{k}(V, \mathbb{Z})$ for all $k \leqslant 2$. It 


\section{INTEGRAL COHOMOLOGY OF THE GENERALIZED KUMMER FOURFOLD}

remains to consider the following exact sequence:

$$
0 \longrightarrow H^{3}(A \times A, \mathbb{Z}) \longrightarrow H^{3}(V, \mathbb{Z}) \longrightarrow H^{4}(A \times A, V, \mathbb{Z}) \stackrel{\varrho}{\longrightarrow} H^{4}(A \times A, \mathbb{Z}) .
$$

The map $\varrho$ is given by $\mathbb{Z}[\Delta] \rightarrow H^{4}(A \times A, \mathbb{Z})$. Using Notation 2.2 , the class $x \otimes 1$ is also in $H^{4}(A \times A, \mathbb{Z})$ and intersects $\Delta$ in one point. Hence, the class of $\Delta$ in $H^{4}(A \times A, \mathbb{Z})$ is not trivial and the map $\varrho$ is injective. It follows that $H^{3}(A \times A, \mathbb{Z})=H^{3}(V, \mathbb{Z})$.

We now calculate the invariants $l_{1,-}^{2}(A \times A)$ and $l_{1,+}^{1}(A \times A)$ from Definition-Proposition 2.3. Lemma 2.9. We have $l_{1,+}^{1}(A \times A)=0$ and $l_{1,-}^{2}(A \times A)=b_{1}(A)$.

Proof. By the Künneth formula, we have

$$
H^{1}(A \times A, \mathbb{Z})=H^{0}(A, \mathbb{Z}) \otimes H^{1}(A, \mathbb{Z}) \oplus H^{1}(A, \mathbb{Z}) \otimes H^{0}(A, \mathbb{Z}) .
$$

The elements of $H^{0}(A, \mathbb{Z}) \otimes H^{1}(A, \mathbb{Z})$ and $H^{1}(A, \mathbb{Z}) \otimes H^{0}(A, \mathbb{Z})$ are exchanged under the action of $\sigma_{2}$. It follows that $l_{2}^{1}(A \times A)=b_{1}(A)$ and, necessarily, $l_{1,-}^{1}(A \times A)=l_{1,+}^{1}(A \times A)=0$. Using the Künneth formula again, we get

$$
H^{2}(A \times A, \mathbb{Z})=H^{0}(A, \mathbb{Z}) \otimes H^{2}(A, \mathbb{Z}) \oplus H^{1}(A, \mathbb{Z}) \otimes H^{1}(A, \mathbb{Z}) \oplus H^{2}(A, \mathbb{Z}) \otimes H^{0}(A, \mathbb{Z}) .
$$

As before, the elements of $H^{0}(A, \mathbb{Z}) \otimes H^{2}(A, \mathbb{Z})$ and $H^{2}(A, \mathbb{Z}) \otimes H^{0}(A, \mathbb{Z})$ are exchanged under the action of $\sigma_{2}$. Furthermore, the elements $z \otimes y \in H^{1}(A, \mathbb{Z}) \otimes H^{1}(A, \mathbb{Z})$ are sent to $-y \otimes z$ by the action of $\sigma_{2}$. Such an element is anti-invariant under the action of $\sigma_{2}$ if $z=y$. It follows that

$$
l_{2}^{2}(A \times A)=b_{2}(A)+\frac{b_{1}(A)\left(b_{1}(A)-1\right)}{2}, \quad l_{1,-}^{2}(A \times A)=b_{1}(A),
$$

and thus $l_{1,+}^{2}(A \times A)=0$.

Lemma 2.10. The torsion part of the group $H^{3}(U, \mathbb{Z})$ is isomorphic to $(\mathbb{Z} / 2 \mathbb{Z})^{\oplus b_{1}(A)}$.

Proof. Using the spectral sequence of equivariant cohomology, this follows from [Men14, Proposition 3.2.5] and Lemmas 2.8 and 2.9.

\subsection{Third cohomology group}

By [Voi02, Theorem 7.31], we have

$$
H^{3}\left(\mathrm{Bl}_{\Delta}(A \times A), \mathbb{Z}\right)=b^{*}\left(H^{3}(A \times A, \mathbb{Z})\right) \oplus j_{*} b_{\mid E}^{*}\left(H^{1}(\Delta, \mathbb{Z})\right) .
$$

It follows that

$$
H^{3}\left(A^{[2]}, \mathbb{Z}\right) \supset \pi_{*} b^{*}\left(H^{3}(A \times A, \mathbb{Z})\right) \oplus \pi_{*} j_{*} b_{\mid E}^{*}\left(H^{1}(\Delta, \mathbb{Z})\right) .
$$

We want to find an equality. We will proceed as follows: We first prove that $\pi_{*} b^{*}\left(H^{3}(A \times A, \mathbb{Z})\right)$ is primitive. Then, in Lemma 2.11, we show that all the elements of $\pi_{*} j_{*} b_{\mid E}^{*}\left(H^{1}(\Delta, \mathbb{Z})\right)$ are divisible by 2 , and, finally, we remark that this implies that the direct sum $\pi_{*} b^{*}\left(H^{3}(A \times A, \mathbb{Z})\right) \oplus$ $\frac{1}{2} \pi_{*} j_{*} b_{\mid E}^{*}\left(H^{1}(\Delta, \mathbb{Z})\right)$ is primitive.

It follows from the Künneth formula that all elements of $H^{3}(A \times A, \mathbb{Z})^{\mathfrak{S}_{2}}$ are written as $y+\sigma_{2}^{*}(y)$ with $y \in H^{3}(A \times A, \mathbb{Z})$. Since $\frac{1}{2} \pi_{*}\left(y+\sigma_{2}^{*}(y)\right)=\pi_{*}(y)$, it follows that $\pi_{*}\left(b^{*}\left(H^{3}(A \times A, \mathbb{Z})\right)\right)$ is primitive in $H^{3}\left(A^{[2]}, \mathbb{Z}\right)$. Moreover, by $(2.2)$, we have the following values, which will be used in Section 2.4:

$$
l_{2}^{3}\left(\mathrm{Bl}_{\Delta}(A \times A)\right)=\operatorname{rk} H^{3}(A \times A, \mathbb{Z})^{\mathfrak{S}_{2}}=b_{1}(A)\left(b_{2}(A)+1\right)
$$




\section{S. Kapfer and G. Menet}

and

$$
l_{1,+}^{3}\left(\mathrm{Bl}_{\Delta}(A \times A)\right)=\operatorname{rk} H^{1}(\Delta, \mathbb{Z})^{\mathfrak{S}_{2}}=b_{1}(A) \quad \text { and } \quad l_{1,-}^{3}\left(\mathrm{Bl}_{\Delta}(A \times A)\right)=0 .
$$

Lemma 2.11. All the elements of the group $\pi_{*} j_{*} b_{\mid E}^{*}\left(H^{1}(\Delta, \mathbb{Z})\right)$ are divisible by 2 in $H^{3}\left(A^{[2]}, \mathbb{Z}\right)$.

Proof. We consider the following commutative diagram:

$$
\begin{gathered}
H^{3}\left(\mathscr{N}_{A^{[2]} / \pi(E)}, \mathscr{N}_{A^{[2]} / \pi(E)} \backslash 0, \mathbb{Z}\right)=H^{3}\left(A^{[2]}, U, \mathbb{Z}\right) \stackrel{g}{\longrightarrow} H^{3}\left(A^{[2]}, \mathbb{Z}\right) \\
\downarrow d \pi^{*} \\
H^{3}\left(\mathscr{N}_{\mathrm{Bl}_{\Delta}(A \times A) / E}, \mathscr{N}_{\mathrm{Bl}_{\Delta}(A \times A) / E} \backslash 0, \mathbb{Z}\right)=H^{3}\left(\mathrm{Bl}_{\Delta}(A \times A), V, \mathbb{Z}\right) \stackrel{h}{\rightarrow} H^{3}\left(\mathrm{Bl}_{\Delta}(A \times A), \mathbb{Z}\right),
\end{gathered}
$$

where $\mathscr{N}_{A^{[2]} / E}$ and $\mathscr{N}_{\mathrm{Bl}_{\Delta}(A \times A) / E}$ are the normal bundles of $\pi(E)$ in $A^{[2]}$ and of $E$ in $\mathrm{Bl}_{\Delta}(A \times$ $A)$, respectively. By the proof of [Voi02, Theorem 7.31], the map $h$ is injective with image in $H^{3}\left(\mathrm{Bl}_{\Delta}(A \times A), \mathbb{Z}\right)$ given by $j_{*} b_{\mid E}^{*}\left(H^{1}(\Delta, \mathbb{Z})\right)$. Hence, diagram (2.4) shows that $g$ is also injective and has image $\pi_{*} j_{*} b_{\mid E}^{*}\left(H^{1}(\Delta, \mathbb{Z})\right)$ in $H^{3}\left(A^{[2]}, \mathbb{Z}\right)$. We obtain

$$
0 \longrightarrow H^{3}\left(A^{[2]}, U, \mathbb{Z}\right) \stackrel{g}{\longrightarrow} H^{3}\left(A^{[2]}, \mathbb{Z}\right) \longrightarrow H^{3}(U, \mathbb{Z}) .
$$

From the Thom isomorphism, we know that $H^{4}\left(A^{[2]}, U, \mathbb{Z}\right)$ is torsion free; hence tors $(\operatorname{coker} g)=$ $\operatorname{tors}\left(H^{3}(U, \mathbb{Z})\right)$, where tors means the torsion part of the groups. It follows, by Lemma 2.10, that

$$
\operatorname{tors}(\operatorname{coker} g)=(\mathbb{Z} / 2 \mathbb{Z})^{\oplus b_{1}(A)} \text {. }
$$

Since $\mathrm{rk} \pi_{*} j_{*} b_{\mid E}^{*}\left(H^{1}(\Delta, \mathbb{Z})\right)=b_{1}(A)$, it follows that all elements of $\pi_{*} j_{*} b_{\mid E}^{*}\left(H^{1}(\Delta, \mathbb{Z})\right)$ are divisible by 2 in $H^{3}\left(A^{[2]}, \mathbb{Z}\right)$.

Now, it remains to prove that $\pi_{*} b^{*}\left(H^{3}(A \times A, \mathbb{Z})\right) \oplus \frac{1}{2} \pi_{*} j_{*} b_{\mid E}^{*}\left(H^{1}(\Delta, \mathbb{Z})\right)$ is primitive in $H^{3}\left(A^{[2]}, \mathbb{Z}\right)$. This comes from the fact that all elements in $\pi_{*} b^{*}\left(H^{3}\left(\mathrm{Bl}_{\Delta}(A \times A), \mathbb{Z}\right)^{\mathfrak{S}_{2}}\right)$ are divisible by 2 , so the relations (2.1) on $\pi_{*}$ and $\pi^{*}$ impose that the above sum be primitive.

More precisely, let $y \in \pi_{*} b^{*}\left(H^{3}(A \times A, \mathbb{Z})\right)$ and $z \in \pi_{*} j_{*} b_{\mid E}^{*}\left(H^{1}(\Delta, \mathbb{Z})\right)$. It is enough to show that if $\frac{1}{2}(y+z) \in H^{3}\left(A^{[2]}, \mathbb{Z}\right)$, then $\frac{1}{2} y \in H^{3}\left(A^{[2]}, \mathbb{Z}\right)$ and $\frac{1}{2} z \in H^{3}\left(A^{[2]}, \mathbb{Z}\right)$. As we have seen, we can write $y=\frac{1}{2} \pi_{*}\left(w+\sigma_{2}^{*}(w)\right)$ with $w \in b^{*}\left(H^{3}(A \times A, \mathbb{Z})\right)$ and $z=\frac{1}{2} \pi_{*}\left(z^{\prime}\right)$ with $z^{\prime} \in j_{*} b_{\mid E}^{*}\left(H^{1}(\Delta, \mathbb{Z})\right)$. If

$$
\frac{\frac{1}{2} \pi_{*}\left(w+\sigma_{2}^{*}(w)\right)+\frac{1}{2} \pi_{*}\left(z^{\prime}\right)}{2} \in H^{3}\left(A^{[2]}, \mathbb{Z}\right),
$$

then taking the image by $\pi^{*}$ of this element, we obtain

$$
\frac{w+\sigma_{2}^{*}(w)+z^{\prime}}{2} \in H^{3}\left(\mathrm{Bl}_{\Delta}(A \times A), \mathbb{Z}\right) .
$$

Hence from (2.2), necessarily $z^{\prime}$ is divisible by 2 . It follows that $z$ is divisible by 2 and so is $y$.

This finishes the proof of part (i) of Proposition 2.1.

\subsection{The fifth cohomology group}

We now prove part (ii) of Proposition 2.1. We will need two basic properties from lattice theory that we recall here, which can be found, for example, in [Dol12, Chapter 8.2.1]. 


\section{INTEGRAL COHOMOLOGY OF THE GENERALIZED KUMMER FOURFOLD}

Let $M$ be a lattice. Let $L \subset M$ be a sublattice of the same rank. Then

$$
|M: L|=\sqrt{\frac{\operatorname{discr} L}{\operatorname{discr} M}} .
$$

We recall that the discriminant $\operatorname{discr} L$ of a lattice $L$ is defined by the absolute value of the determinant of the matrix of its bilinear form.

If $M$ is unimodular and $L \subset M$ is a primitive embedding, then

$$
\operatorname{discr} L=\operatorname{discr} L^{\perp} \text {. }
$$

By [Voi02, Theorem 7.31], we have

$$
H^{5}\left(\mathrm{Bl}_{\Delta}(A \times A), \mathbb{Z}\right)=b^{*}\left(H^{5}(A \times A, \mathbb{Z})\right) \oplus j_{*} b_{\mid E}^{*}\left(H^{3}(\Delta, \mathbb{Z})\right) .
$$

It follows that

$$
H^{5}\left(A^{[2]}, \mathbb{Z}\right) \supset \pi_{*}\left(b^{*}\left(H^{5}(A \times A, \mathbb{Z})\right)\right) \oplus \pi_{*} j_{*} b_{\mid E}^{*}\left(H^{3}(\Delta, \mathbb{Z})\right) .
$$

As before, by looking at the Künneth formula, we see that $\pi_{*}\left(b^{*}\left(H^{5}(A \times A, \mathbb{Z})\right)\right)$ is primitive in $H^{5}\left(A^{[2]}, \mathbb{Z}\right)$. Moreover, by $(2.8)$, we have

$$
l_{2}^{5}\left(\mathrm{Bl}_{\Delta}(A \times A)\right)=\operatorname{rk} H^{5}(A \times A, \mathbb{Z})^{\mathfrak{S}_{2}}=b_{1}(A)\left(b_{2}(A)+1\right)
$$

and

$$
l_{1,+}^{5}\left(\mathrm{Bl}_{\Delta}(A \times A)\right)=\operatorname{rk} H^{3}(\Delta, \mathbb{Z})^{\mathfrak{S}_{2}}=b_{1}(A) \quad \text { and } \quad l_{1,-}^{5}\left(\mathrm{Bl}_{\Delta}(A \times A)\right)=0 .
$$

Lemma 2.12. The lattice $\pi_{*}\left(H^{3}\left(\mathrm{Bl}_{\Delta}(A \times A), \mathbb{Z}\right) \oplus H^{5}\left(\mathrm{Bl}_{\Delta}(A \times A), \mathbb{Z}\right)\right)$ has discriminant $2^{2 b_{1}(A)}$. Proof. By Proposition 2.5(ii), the quotient

$$
\begin{aligned}
& \frac{H^{3}\left(\mathrm{Bl}_{\Delta}(A \times A), \mathbb{Z}\right) \oplus H^{5}\left(\mathrm{Bl}_{\Delta}(A \times A), \mathbb{Z}\right)}{H^{3}\left(\mathrm{Bl}_{\Delta}(A \times A), \mathbb{Z}\right)^{\mathfrak{S}_{2}} \oplus H^{5}\left(\mathrm{Bl}_{\Delta}(A \times A), \mathbb{Z}\right)^{\mathfrak{S}_{2}} \oplus\left(H^{3}\left(\mathrm{Bl}_{\Delta}(A \times A), \mathbb{Z}\right)^{\mathfrak{S}_{2}} \oplus H^{5}\left(\mathrm{Bl}_{\Delta}(A \times A), \mathbb{Z}\right)^{\mathfrak{S}_{2}}\right)^{\perp}} \\
& \text { equals }(\mathbb{Z} / 2 \mathbb{Z})^{\oplus\left(l_{2}^{3}\left(\mathrm{Bl}_{\Delta}(A \times A)\right)+l_{2}^{5}\left(\mathrm{Bl}_{\Delta}(A \times A)\right)\right) .}
\end{aligned}
$$

Since $H^{3}\left(\mathrm{Bl}_{\Delta}(A \times A), \mathbb{Z}\right) \oplus H^{5}\left(\mathrm{Bl}_{\Delta}(A \times A), \mathbb{Z}\right)$ is a unimodular lattice, it follows from (2.7) and (2.6) that

$$
\operatorname{discr}\left[H^{3}\left(\mathrm{Bl}_{\Delta}(A \times A), \mathbb{Z}\right)^{\mathfrak{S}_{2}} \oplus H^{5}\left(\mathrm{Bl}_{\Delta}(A \times A), \mathbb{Z}\right)^{\mathfrak{S}_{2}}\right]=2^{l_{2}^{3}\left(\mathrm{Bl}_{\Delta}(A \times A)\right)+l_{2}^{5}\left(\mathrm{Bl}_{\Delta}(A \times A)\right)} .
$$

Then, by Proposition 2.7, we have

$$
\begin{aligned}
& \operatorname{discr} \pi_{*}\left(H^{3}\left(\mathrm{Bl}_{\Delta}(A \times A), \mathbb{Z}\right)^{\mathfrak{S}_{2}} \oplus H^{5}\left(\mathrm{Bl}_{\Delta}(A \times A), \mathbb{Z}\right)^{\mathfrak{S}_{2}}\right)
\end{aligned}
$$

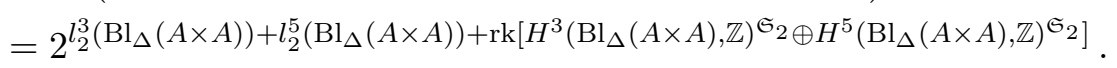

Next, by Proposition 2.5(i), we have

$$
\begin{aligned}
& \operatorname{discr} \pi_{*}\left(H^{3}\left(\mathrm{Bl}_{\Delta}(A \times A), \mathbb{Z}\right)^{\mathfrak{S}_{2}} \oplus H^{5}\left(\mathrm{Bl}_{\Delta}(A \times A), \mathbb{Z}\right)^{\mathfrak{S}_{2}}\right) \\
& \quad=2^{2\left(l_{2}^{3}\left(\mathrm{Bl}_{\Delta}(A \times A)\right)+l_{2}^{5}\left(\mathrm{Bl}_{\Delta}(A \times A)\right)\right)+l_{1,+}^{3}\left(\mathrm{Bl}_{\Delta}(A \times A)\right)+l_{1,+}^{5}\left(\mathrm{Bl}_{\Delta}(A \times A)\right) .}
\end{aligned}
$$

By Remark 2.6 and the equality $\pi_{*}\left(x+\iota^{*}(x)\right)=2 \pi_{*}(x)$, we have

$$
\frac{\pi_{*}\left(H^{3}\left(\mathrm{Bl}_{\Delta}(A \times A), \mathbb{Z}\right) \oplus H^{5}\left(\mathrm{Bl}_{\Delta}(A \times A), \mathbb{Z}\right)\right)}{\pi_{*}\left(H^{3}\left(\mathrm{Bl}_{\Delta}(A \times A), \mathbb{Z}\right)^{\mathfrak{S}_{2}} \oplus H^{5}\left(\mathrm{Bl}_{\Delta}(A \times A), \mathbb{Z}\right)^{\mathfrak{S}_{2}}\right)}=\left(\frac{\mathbb{Z}}{2 \mathbb{Z}}\right)^{\oplus\left(l_{2}^{3}\left(\mathrm{Bl}_{\Delta}(A \times A)\right)+l_{2}^{5}\left(\mathrm{Bl}_{\Delta}(A \times A)\right)\right)}
$$


Then by (2.10), (2.6), (2.3) and (2.9), we have

$$
\begin{aligned}
& \operatorname{discr} \pi_{*}\left(H^{3}\left(\mathrm{Bl}_{\Delta}(A \times A), \mathbb{Z}\right) \oplus H^{5}\left(\mathrm{Bl}_{\Delta}(A \times A), \mathbb{Z}\right)\right) \\
& =2^{l_{1,+}^{3}\left(\mathrm{Bl}_{\Delta}(A \times A)\right)+l_{1,+}^{5}\left(\mathrm{Bl}_{\Delta}(A \times A)\right)}=2^{2 b_{1}(A)} .
\end{aligned}
$$

The lattice $H^{3}\left(A^{[2]}, \mathbb{Z}\right) \oplus H^{5}\left(A^{[2]}, \mathbb{Z}\right)$ is unimodular. Hence, by (2.6), we have

$$
\frac{H^{3}\left(A^{[2]}, \mathbb{Z}\right) \oplus H^{5}\left(A^{[2]}, \mathbb{Z}\right)}{\pi_{*}\left(H^{3}\left(\mathrm{Bl}_{\Delta}(A \times A), \mathbb{Z}\right) \oplus H^{5}\left(\mathrm{Bl}_{\Delta}(A \times A), \mathbb{Z}\right)\right)}=\left(\frac{\mathbb{Z}}{2 \mathbb{Z}}\right)^{\oplus b_{1}(A)} .
$$

However, from the last section, we know that

$$
H^{3}\left(A^{[2]}, \mathbb{Z}\right) / \pi_{*}\left(H^{3}\left(\mathrm{Bl}_{\Delta}(A \times A), \mathbb{Z}\right)\right)=(\mathbb{Z} / 2 \mathbb{Z})^{\oplus b_{1}(A)} .
$$

It follows that $H^{5}\left(A^{[2]}, \mathbb{Z}\right) / \pi_{*}\left(H^{5}\left(\mathrm{Bl}_{\Delta}(A \times A), \mathbb{Z}\right)\right)=0$, which proves Proposition 2.1(ii).

\section{Nakajima operators for Hilbert schemes of points on surfaces}

Let $A$ be a smooth projective complex surface. Let $A^{[n]}$ the Hilbert scheme of $n$ points on the surface, that is, the moduli space of finite subschemes of $A$ of length $n$. The scheme $A^{[n]}$ is again smooth and projective of dimension $2 n$; see [Fog68]. The rational cohomology of $A^{[n]}$ can be described in terms of Nakajima's operators [Nak97]. First, consider the direct sum

$$
\mathbb{H}:=\bigoplus_{n=0}^{\infty} H^{*}\left(A^{[n]}, \mathbb{Q}\right)
$$

This space is bigraded by the cohomological degree and the weight, which is given by the number of points $n$. The unit element in $H^{0}\left(A^{[0]}, \mathbb{Q}\right) \cong \mathbb{Q}$ is denoted by $|0\rangle$ and is called the vacuum.

Definition-Proposition 3.1. There are linear operators $\mathfrak{q}_{m}(a)$, for each $m \geqslant 1$ and $a \in$ $H^{*}(A, \mathbb{Q})$, acting on $\mathbb{H}$, which have the following properties: they depend linearly on $a$, and if $a \in H^{k}(A, \mathbb{Q})$ is homogeneous, the operator $\mathfrak{q}_{m}(a)$ is bihomogeneous of degree $k+2(m-1)$ and weight $m$ :

$$
\mathfrak{q}_{m}(a): H^{l}\left(A^{[n]}\right) \rightarrow H^{l+k+2(m-1)}\left(A^{[n+m]}\right) .
$$

To construct these operators, first define incidence varieties $\mathcal{Z}_{m} \subset A^{[n]} \times A \times A^{[n+m]}$ by

$$
\mathcal{Z}_{m}:=\left\{\left(\xi, x, \xi^{\prime}\right) \mid \xi \subset \xi^{\prime}, \operatorname{supp}\left(\xi^{\prime}\right)-\operatorname{supp}(\xi)=m x\right\} .
$$

Then $\mathfrak{q}_{m}(a)(\beta)$ is defined as the Poincaré dual of $\operatorname{pr}_{3 *}\left(\left(\operatorname{pr}_{2}^{*}(\alpha) \cdot \operatorname{pr}_{1}^{*}(\beta)\right) \cap\left[\mathcal{Z}_{m}\right]\right)$.

Now, consider the superalgebra generated by the $\mathfrak{q}_{m}(a)$. Every element in $\mathbb{H}$ can be decomposed uniquely as a linear combination of products of operators $\mathfrak{q}_{m}(a)$ acting on the vacuum. In other words, the $\mathfrak{q}_{m}(a)$ generate $\mathbb{H}$ and there are no algebraic relations between them (except the linearity in $a$ and the supercommutativity).

DeFinition 3.2. To give the cup product structure of $\mathbb{H}$, define operators $\mathfrak{G}(a)$ for $a \in H^{*}(A)$. Let $\Xi_{n} \subset A^{[n]} \times A$ be the universal subscheme. Then the action of $\mathfrak{G}(a)$ on $H^{*}\left(A^{[n]}\right)$ is multiplication with the class

$$
\operatorname{pr}_{1 *}\left(\operatorname{ch}\left(\mathcal{O}_{\Xi_{n}}\right) \cdot \operatorname{pr}_{2}^{*}(\operatorname{td}(A) \cdot a)\right) \in H^{*}\left(A^{[n]}\right) .
$$

For $a \in H^{k}(A)$, we define $\mathfrak{G}_{i}(a)$ as the component of $\mathfrak{G}(a)$ of cohomological degree $k+2 i$. A differential operator $\mathfrak{d}$ is given by $\mathfrak{G}_{1}(1)$, which stands for multiplication with the first Chern class of the tautological sheaf $\operatorname{pr}_{1 *}\left(\mathcal{O}_{\Xi_{n}}\right)$. 


\section{INTEGRAL COHOMOLOGY OF THE GENERALIZED KUMMER FOURFOLD}

In [LS03] and [LQW02b], we find various commutation relations between these operators, that allow us to determine all multiplications in the cohomology of the Hilbert scheme. First of all, if $X$ and $Y$ are operators of degrees $d$ and $d^{\prime}$, respectively, their commutator is defined in the supersense:

$$
[X, Y]:=X Y-(-1)^{d d^{\prime}} Y X .
$$

The integral on $A^{[n]}$ induces a non-degenerate bilinear form on $\mathbb{H}$ : for classes $\alpha, \beta \in H^{*}\left(A^{[n]}\right)$, it is given by

$$
(\alpha, \beta)_{A^{[n]}}:=\int_{A^{[n]}} \alpha \cdot \beta .
$$

If $X$ is a homogeneous linear operator of degree $d$ and weight $m$ acting on $\mathbb{H}$, we define its adjoint $X^{\dagger}$ by

$$
(X(\alpha), \beta)_{A^{[n+m]}}=(-1)^{d|\alpha|}\left(\alpha, X^{\dagger}(\beta)\right)_{A^{[n]}} .
$$

We put $\mathfrak{q}_{0}(a):=0$ and $\mathfrak{q}_{m}(a):=(-1)^{m} \mathfrak{q}_{-m}(a)^{\dagger}$ for $m<0$. Note that for all $m \in \mathbb{Z}$, the bidegree of $\mathfrak{q}_{m}(a)$ is $(m,|a|+2(|m|-1))$. If $m$ is positive, $\mathfrak{q}_{m}$ is called a creation operator; otherwise, it is called an annihilation operator. Now, define

$$
\mathfrak{L}_{m}(a):= \begin{cases}\frac{1}{2} \sum_{k \in \mathbb{Z}} \sum_{i} \mathfrak{q}_{k}\left(a_{(1)}\right) \mathfrak{q}_{m-k}\left(a_{(2)}\right) & \text { if } m \neq 0, \\ \sum_{k>0} \sum_{i} \mathfrak{q}_{k}\left(a_{(1)}\right) \mathfrak{q}_{-k}\left(a_{(2)}\right) & \text { if } m=0,\end{cases}
$$

where $\sum_{i} a_{(1)} \otimes a_{(2)}$ is the push-forward of $a$ along the diagonal $\tau_{2}: A \rightarrow A \times A$ (in Sweedler's notation).

Lemma 3.3 ([LQW02b, Theorem 2.16]). Let $K_{A} \in H^{2}(A, \mathbb{Q})$ be the class of the canonical divisor. We have

$$
\begin{aligned}
{\left[\mathfrak{q}_{m}(a), \mathfrak{q}_{n}(b)\right] } & =m \cdot \delta_{m+n} \cdot \int_{A} a b \\
{\left[\mathfrak{L}_{m}(a), \mathfrak{q}_{n}(b)\right] } & =-n \cdot \mathfrak{q}_{m+n}(a b), \\
{\left[\mathfrak{d}, \mathfrak{q}_{m}(a)\right] } & =m \cdot \mathfrak{L}_{m}(a)+\frac{1}{2} m(|m|-1) \mathfrak{q}_{m}\left(K_{A} a\right), \\
{\left[\mathfrak{G}_{k}(a), \mathfrak{q}_{1}(b)\right] } & =(1 / k !) \operatorname{ad}(\mathfrak{d})^{k}\left(\mathfrak{q}_{1}(a b)\right) .
\end{aligned}
$$

Remark 3.4. Note (cf. [LS03, Theorem 3.8]) that (3.2) together with (3.3) imply that

$$
\mathfrak{q}_{m+1}(a)=\frac{(-1)^{m}}{m !}\left(\operatorname{ad} \mathfrak{q}^{\prime}\right)^{m}\left(\mathfrak{q}_{1}(a)\right),
$$

so that there are two ways of writing an element of $\mathbb{H}$ : as a linear combination of products of creation operators $\mathfrak{q}_{m}(a)$ or as a linear combination of products of the operators $\mathfrak{d}$ and $\mathfrak{q}_{1}(a)$. This second representation is more suitable for computing cup-products but not faithful. Equations (3.3) and (3.5) now permit us to switch between the two representations.

Remark 3.5. We have adopted the notation from [LQW02b], which differs from the conventions in [LS03]. Here is part of a dictionary: 


\section{S. Kapfer and G. Menet}

\begin{tabular}{c|c} 
Notation from [LQW02b] & Notation from [LS03] \\
\hline operator of weight $w$ and degree $d$ & operator of weight $w$ and degree $d-2 w$ \\
$\mathfrak{q}_{m}(a)$ & $\mathfrak{p}_{-m}(a)$ \\
$\mathfrak{L}_{m}(a)$ & $-L_{-m}(a)$ \\
$\mathfrak{G}(a)$ & $a^{[\bullet]}$ \\
$\mathfrak{d}$ & $\partial$ \\
$\tau_{2 *}(a)$ & $-\Delta(a)$
\end{tabular}

By sending a subscheme in $A$ to its support, we define a morphism

$$
\varrho: A^{[n]} \longrightarrow \operatorname{Sym}^{n}(A),
$$

called the Hilbert-Chow morphism. The cohomology of $\operatorname{Sym}^{n}(A)$ is given by elements of the $n$ fold tensor power of $H^{*}(A)$ that are invariant under the action of the group of permutations $\mathfrak{S}_{n}$. A class in $H^{*}\left(A^{[n]}, \mathbb{Q}\right)$ which can be written using only the operators $\mathfrak{q}_{1}(a)$ of weight 1 comes from a pullback along $\varrho$ :

$$
\mathfrak{q}_{1}\left(b_{1}\right) \cdots \mathfrak{q}_{1}\left(b_{n}\right)|0\rangle=\varrho^{*}\left(\sum_{\pi \in \mathfrak{S}_{n}} \pm b_{\pi(1)} \otimes \cdots \otimes b_{\pi(n)}\right), \quad b_{i} \in H^{*}(A, \mathbb{Q}),
$$

where signs arise from permuting factors of odd degrees. In particular,

$$
\begin{aligned}
\frac{1}{n !} \mathfrak{q}_{1}(b)^{n}|0\rangle & =\varrho^{*}(b \otimes \cdots \otimes b), \\
\frac{1}{(n-1) !} \mathfrak{q}_{1}(b) \mathfrak{q}^{n-1}|0\rangle & =\varrho^{*}(b \otimes 1 \otimes \cdots \otimes 1+\cdots+1 \otimes \cdots \otimes 1 \otimes b) .
\end{aligned}
$$

\section{On the integral cohomology of Hilbert schemes}

For the study of integral cohomology, first note that if $a \in H^{*}(A, \mathbb{Z})$ is an integral class, then $\mathfrak{q}_{m}(a)$ maps integral classes to integral classes. Operators satisfying this property are called integral. Qin and Wang studied them in [QW05]. We need the following results.

Lemma 4.1 ([QW05, Lemmas 3.3 and 3.6 and Theorem 4.5]). The operators $\frac{1}{n !} \mathfrak{q}_{1}(1)^{n}$ and $\frac{1}{2} \mathfrak{q}_{2}(1)$ are integral. Let $b \in H^{2}(A, \mathbb{Z})$ be monodromy equivalent to a divisor. Then the operator $\frac{1}{2} \mathfrak{q}_{1}(b)^{2}-$ $\frac{1}{2} \mathfrak{q}_{2}(b)$ is integral.

Remark 4.2. Qin and Wang [QW05, Theorem 1.1 et seq.] conjecture that this works even without the restriction on $b \in H^{2}(A, \mathbb{Z})$.

Corollary 4.3. If $A$ is a torus, the operator $\frac{1}{2} \mathfrak{q}_{1}(b)^{2}-\frac{1}{2} \mathfrak{q}_{2}(b)$ is integral for all $b \in H^{2}(A, \mathbb{Z})$.

Proof. The Nakajima operators are preserved under deformations of $A$. Moreover, the image $\operatorname{Mon}(A)$ of the monodromy representation on $H^{2}(A, \mathbb{Z})$ is given by $O^{+,+}\left(H^{2}(A, \mathbb{Z})\right)$, the group of isometries on $H^{2}(A, \mathbb{Z})$ preserving the orientation of the negative- and positive-definite parts of $H^{2}(A, \mathbb{R})$. Indeed, by the last remark in [Bor86], the subgroup $\operatorname{Diff}(A)$ of $O\left(H^{2}(A, \mathbb{Z})\right)$ induced by the diffeomorphisms of $A$ is equal to $O^{+,+}\left(H^{2}(A, \mathbb{Z})\right)$. Hence, $\operatorname{Mon}(A) \subset \operatorname{Diff}(A)=$ $O^{+,+}\left(H^{2}(A, \mathbb{Z})\right)$. Furthermore, by [Shi78, Section 4, Theorem 1 and Section 5, Theorem 2], the moduli space of marked complex tori has four connected components. It follows that, necessarily, $\operatorname{Mon}(A)$ has at most index 4 in $O\left(H^{2}(A, \mathbb{Z})\right)$. So $\operatorname{Mon}(A)=O^{+,+}\left(H^{2}(A, \mathbb{Z})\right)$.

Now, suppose that the Néron-Severi group NS $(A)$ contains a copy of the hyperbolic lattice $U$ (such $A$ exist). Let us write $H^{2}(A, \mathbb{Z})=U_{1} \oplus U_{2} \oplus U_{3}$ with $\mathrm{NS}(A)=U_{1}$ and $U_{i}$ isometric to $U$ for 


\section{INTEGRAL COHOMOLOGY OF THE GENERALIZED KUMMER FOURFOLD}

all $i \in\{1,2,3\}$. We consider two isometries $\varphi_{2}$ and $\varphi_{3}$ in $O^{+,+}\left(H^{2}(A, \mathbb{Z})\right)$, defined in the following way: $\varphi_{2}$ exchanges $U_{1}$ and $U_{2}$ and acts as - id on $U_{3}$, and $\varphi_{3}$ exchanges $U_{1}$ and $U_{3}$ and acts as - id on $U_{2}$. Through these two isometries, all elements of $U_{2}$ and $U_{3}$ are monodromy equivalent to a divisor. Then Lemma 4.1 establishes the corollary for that particular $A$. Now, since all tori are equivalent by deformation, a general torus can always be deformed to our special $A$. Since the integrality of an operator is a topological invariant, $\frac{1}{2} \mathfrak{q}_{1}(b)^{2}-\frac{1}{2} \mathfrak{q}_{2}(b)$ remains integral for all $b \in H^{2}(A, \mathbb{Z})$.

Proposition 4.4. Assume that $H^{*}(A, \mathbb{Z})$ is torsion free. We are using Notation 2.2. Denote the dual element to $b_{i} \in H^{2}(A, \mathbb{Z})$ by $b_{i}^{*}$. Modulo torsion, the following classes form a basis of $H^{2}\left(A^{[n]}, \mathbb{Z}\right)$ :

$$
\begin{aligned}
& \frac{1}{(n-1) !} \mathfrak{q}_{1}\left(b_{i}\right) \mathfrak{q}_{1}(1)^{n-1}|0\rangle=\mathfrak{G}_{0}\left(b_{i}\right) 1, \\
& \frac{1}{(n-2) !} \mathfrak{q}_{1}\left(a_{i}\right) \mathfrak{q}_{1}\left(a_{j}\right) \mathfrak{q}_{1}(1)^{n-2}|0\rangle=\mathfrak{G}_{0}\left(a_{i}\right) \mathfrak{G}_{0}\left(a_{j}\right) 1 \text { for } i<j, \\
& \frac{1}{2(n-2) !} \mathfrak{q}_{2}(1) \mathfrak{q}_{1}(1)^{n-2}|0\rangle \quad(\text { denote this class by } \delta) .
\end{aligned}
$$

Their respective duals in $H^{2 n-2}\left(A^{[n]}, \mathbb{Z}\right)$ are given by

$$
\mathfrak{q}_{1}\left(b_{i}^{*}\right) \mathfrak{q}_{1}(x)^{n-1}|0\rangle, \quad \mathfrak{q}_{1}\left(a_{j}^{*}\right) \mathfrak{q}_{1}\left(a_{i}^{*}\right) \mathfrak{q}_{1}(x)^{n-2}|0\rangle \quad \text { for } i<j \quad \mathfrak{q}_{2}(x) \mathfrak{q}_{1}(x)^{n-2}|0\rangle .
$$

Proof. It is clear from the above lemma that these classes are all integral. Göttsche's formula [Göt94, Section 2.3, p. 35] gives the Betti numbers of $A^{[n]}$ in terms of the Betti numbers of $A$, namely $h^{1}\left(A^{[n]}\right)=h^{1}(A)$ and $h^{2}\left(A^{[n]}\right)=h^{2}(A)+\frac{1}{2} h^{1}(A)\left(h^{1}(A)-1\right)+1$. It follows that the given classes span a lattice of full rank.

Next we have to show that the intersection matrix between these classes is in fact the identity matrix. Most of the entries can be computed easily using the simplification from (3.7). For products involving $\delta$ (this is the action of $\mathfrak{d}$ ) or its dual, first observe that

$$
\mathfrak{d} \mathfrak{q}_{1}(x)^{m}|0\rangle=0 \quad \text { and } \quad \mathfrak{L}_{1}(a) \mathfrak{q}_{1}(x)^{m}|0\rangle=0
$$

for every class $a$ of degree at least 1. Then compute:

$$
\begin{aligned}
\delta \cdot \mathfrak{q}_{2}(x) \mathfrak{q}_{1}(x)^{n-2}|0\rangle & =\mathfrak{d q}_{2}(x) \mathfrak{q}_{1}(x)^{n-2}|0\rangle=2 \mathfrak{L}_{2}(x) \mathfrak{q}_{1}(x)^{n-2}|0\rangle=\mathfrak{q}_{1}(x)^{n}|0\rangle, \\
\mathfrak{d} \mathfrak{q}_{1}\left(b_{i}^{*}\right) \mathfrak{q}_{1}(x)^{n-1}|0\rangle & =\mathfrak{L}_{1}\left(b_{i}^{*}\right) \mathfrak{q}_{1}(x)^{n-1}|0\rangle=0 \\
\mathfrak{d} \mathfrak{q}_{1}\left(a_{j}^{*}\right) \mathfrak{q}_{1}\left(a_{i}^{*}\right) \mathfrak{q}_{1}(x)^{n-2}|0\rangle & =\left(\mathfrak{L}_{1}\left(a_{j}^{*}\right)+\mathfrak{q}_{1}\left(a_{j}^{*}\right) \mathfrak{d}\right) \mathfrak{q}_{1}\left(a_{i}^{*}\right) \mathfrak{q}_{1}(x)^{n-2}|0\rangle \\
& =\left(-\mathfrak{q}_{1}\left(a_{i}^{*}\right) \mathfrak{L}_{1}\left(a_{j}^{*}\right)+\mathfrak{q}_{1}\left(a_{j}^{*}\right) \mathfrak{L}_{1}\left(a_{i}^{*}\right)\right) \mathfrak{q}_{1}(x)^{n-2}|0\rangle=0, \\
\mathfrak{G}_{0}\left(b_{i}\right) \mathfrak{q}_{2}(x) \mathfrak{q}_{1}(x)^{n-2}|0\rangle & =0 \\
\mathfrak{G}_{0}\left(a_{i}\right) \mathfrak{G}_{0}\left(a_{j}\right) \mathfrak{q}_{2}(x) \mathfrak{q}_{1}(x)^{n-2}|0\rangle & =0
\end{aligned}
$$

Remark 4.5. If $A$ is a complex torus, a theorem of Markman [Mar07] asserts that $H^{*}\left(A^{[n]}, \mathbb{Z}\right)$ is torsion free.

Proposition 4.6. Let $A$ be a complex abelian surface. Using Notation 2.2, a basis of $H^{*}\left(A^{[2]}, \mathbb{Z}\right)$ is given by the classes in the following table: 


\section{S. Kapfer and G. Menet}

\begin{tabular}{|c|c|c|c|}
\hline Degree & Betti number & Class & Multiplication with class \\
\hline 0 & 1 & $\frac{1}{2} \mathfrak{q}_{1}(1)^{2}|0\rangle$ & id \\
\hline 1 & 4 & $\mathfrak{q}_{1}(1) \mathfrak{q}_{1}\left(a_{i}\right)|0\rangle$ & $\mathfrak{G}_{0}\left(a_{i}\right)$ \\
\hline 2 & 13 & $\begin{array}{l}\frac{1}{2} \mathfrak{q}_{2}(1)|0\rangle \\
\mathfrak{q}_{1}\left(a_{i}\right) \mathfrak{q}_{1}\left(a_{j}\right)|0\rangle \text { for } i<j \\
\mathfrak{q}_{1}(1) \mathfrak{q}_{1}\left(b_{i}\right)|0\rangle\end{array}$ & $\begin{array}{l}\mathfrak{d} \\
\mathfrak{G}_{0}\left(a_{i}\right) \mathfrak{G}_{0}\left(a_{j}\right) \\
\mathfrak{G}_{0}\left(b_{i}\right)\end{array}$ \\
\hline 3 & 32 & $\begin{array}{l}\frac{1}{2} \mathfrak{q}_{2}\left(a_{i}\right)|0\rangle \\
\mathfrak{q}_{1}\left(a_{i}\right) \mathfrak{q}_{1}\left(b_{j}\right)|0\rangle \\
\mathfrak{q}_{1}(1) \mathfrak{q}_{1}\left(a_{i}^{*}\right)|0\rangle\end{array}$ & $\begin{array}{l}-\mathfrak{G}_{1}\left(a_{i}\right) \\
\mathfrak{G}_{0}\left(a_{i}\right) \mathfrak{G}_{0}\left(b_{j}\right) \\
\mathfrak{G}_{0}\left(a_{i}^{*}\right)\end{array}$ \\
\hline 4 & 44 & $\begin{array}{l}\left(\frac{1}{2} \mathfrak{q}_{1}\left(b_{i}\right)^{2}-\frac{1}{2} \mathfrak{q}_{2}\left(b_{i}\right)\right)|0\rangle \\
\mathfrak{q}_{1}\left(a_{i}\right) \mathfrak{q}_{1}\left(a_{j}^{*}\right)|0\rangle \\
\mathfrak{q}_{1}\left(b_{i}\right) \mathfrak{q}_{1}\left(b_{j}\right)|0\rangle \text { for } i \leqslant j\end{array}$ & $\begin{array}{l}\frac{1}{2} \mathfrak{G}_{0}\left(b_{i}\right)^{2}+\mathfrak{G}_{1}\left(b_{i}\right) \\
\mathfrak{G}_{0}\left(a_{i}\right) \mathfrak{G}_{0}\left(a_{j}^{*}\right) \\
\mathfrak{G}_{0}\left(b_{i}\right) \mathfrak{G}_{0}\left(b_{j}\right) \\
\end{array}$ \\
\hline 5 & 32 & $\begin{array}{l}\mathfrak{q}_{2}\left(a_{i}^{*}\right)|0\rangle \\
\mathfrak{q}_{1}\left(a_{i}^{*}\right) \mathfrak{q}_{1}\left(b_{j}\right)|0\rangle \\
\mathfrak{q}_{1}\left(a_{i}\right) \mathfrak{q}_{1}(x)|0\rangle\end{array}$ & $\begin{array}{l}-2 \mathfrak{G}_{1}\left(a_{i}^{*}\right) \\
\mathfrak{G}_{0}\left(a_{i}^{*}\right) \mathfrak{G}_{0}\left(b_{j}\right) \\
\mathfrak{G}_{0}\left(a_{i}\right) \mathfrak{G}_{0}(x)\end{array}$ \\
\hline 6 & 13 & $\begin{array}{l}\mathfrak{q}_{2}(x)|0\rangle \\
\mathfrak{q}_{1}\left(a_{i}^{*}\right) \mathfrak{q}_{1}\left(a_{j}^{*}\right)|0\rangle \text { for } i<j \\
\mathfrak{q}_{1}\left(b_{i}\right) \mathfrak{q}_{1}(x)|0\rangle\end{array}$ & $\begin{array}{l}-2 \mathfrak{G}_{1}(x) \\
\mathfrak{G}_{0}\left(a_{i}^{*}\right) \mathfrak{G}_{0}\left(a_{j}^{*}\right) \\
\mathfrak{G}_{0}\left(b_{i}\right) \mathfrak{G}_{0}(x) \\
\end{array}$ \\
\hline 7 & 4 & $\mathfrak{q}_{1}\left(a_{i}^{*}\right) \mathfrak{q}_{1}(x)|0\rangle$ & $\mathfrak{G}_{0}\left(a_{i}^{*}\right) \mathfrak{G}_{0}(x)$ \\
\hline 8 & 1 & $\mathfrak{q}_{1}(x)^{2}|0\rangle$ & $\mathfrak{G}_{0}(x)^{2}$ \\
\hline
\end{tabular}

Proof. The Betti numbers come from Göttsche's formula [Göt94]. One computes the intersection matrix of all classes under the Poincaré duality pairing and finds that it is unimodular. So it remains to show that all these classes are integral. By Lemma 4.1, this is clear for all classes except those of the form $\frac{1}{2} \mathfrak{q}_{2}\left(a_{i}\right)|0\rangle \in H^{3}\left(A^{[2]}, \mathbb{Z}\right)$.

Evaluating the Poincaré duality pairing between degrees 3 and 5 gives

$$
\begin{gathered}
\mathfrak{q}_{2}\left(a_{i}\right)|0\rangle \cdot \mathfrak{q}_{2}\left(a_{i}^{*}\right)|0\rangle=2, \\
\mathfrak{q}_{1}\left(a_{i}\right) \mathfrak{q}_{1}\left(b_{j}\right)|0\rangle \cdot \mathfrak{q}_{1}\left(a_{i}^{*}\right) \mathfrak{q}_{1}\left(b_{j}^{*}\right)|0\rangle=1, \\
\mathfrak{q}_{1}(1) \mathfrak{q}_{1}\left(a_{i}^{*}\right)|0\rangle \cdot \mathfrak{q}_{1}(x) \mathfrak{q}_{1}\left(a_{i}\right)|0\rangle=1,
\end{gathered}
$$

while the other pairings vanish. Therefore, one of $\mathfrak{q}_{2}\left(a_{i}\right)|0\rangle$ and $\mathfrak{q}_{2}\left(a_{i}^{*}\right)|0\rangle$ must be divisible by 2 . With the considerations from Section 2 in mind, we can interpret $\mathfrak{q}_{2}\left(a_{i}\right)|0\rangle \in H^{3}\left(A^{[2]}, \mathbb{Z}\right)$ and $\mathfrak{q}_{2}\left(a_{i}^{*}\right)|0\rangle \in H^{5}\left(A^{[2]}, \mathbb{Z}\right)$ as classes concentrated on the exceptional divisor, that is, as elements of $\pi_{*} j_{*} H^{*}(E, \mathbb{Z})$. Indeed, the push-forward of a class $a \otimes 1 \in H^{k}(E, \mathbb{Z})$ is given by

$$
\pi_{*} j_{*}(a \otimes 1)=\mathfrak{q}_{2}(a)|0\rangle \in H^{k+2}\left(A^{[n]}, \mathbb{Z}\right) .
$$

When pushing forward to the Hilbert scheme, the only possibility to get a factor 2 is in degree 3 , by Proposition 2.1. 


\section{INTEGRAL COHOMOLOGY OF THE GENERALIZED KUMMER FOURFOLD}

\section{Cohomology of generalized Kummer varieties via Hilbert scheme cohomology}

Definition 5.1. Let $A$ be a complex projective torus of dimension 2 and $A^{[n]}$, for $n \geqslant 1$, the corresponding Hilbert scheme of points. Let $\Sigma: A^{[n]} \rightarrow A$ be the summation morphism, a smooth submersion that factors via (3.6), the Hilbert-Chow morphism, giving $A^{[n]} \stackrel{\varrho}{\rightarrow} \operatorname{Sym}^{n}(A) \stackrel{\sigma}{\rightarrow} A$. Then the generalized Kummer variety $K_{n-1}(A)$ is defined as the fibre over 0:

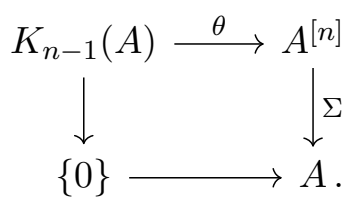

Theorem 5.2 ([Spa56, Theorem 2]). The cohomology of the generalized Kummer variety, the group $H^{*}\left(K_{n-1}(A), \mathbb{Z}\right)$, is torsion free.

Our first objective is to collect some information about the pullback diagram (5.1). We make use of Notation 2.2.

Proposition 5.3. Set $\alpha_{i}:=(1 /(n-1) !) \mathfrak{q}_{1}(1)^{n-1} \mathfrak{q}_{1}\left(a_{i}\right)|0\rangle=\mathfrak{G}_{0}\left(a_{i}\right) 1$. Then the corresponding class of $K_{n-1}(A)$ in $H^{4}\left(A^{[n]}, \mathbb{Z}\right)$ is given by $\left[K_{n-1}(A)\right]=\alpha_{1} \cdot \alpha_{2} \cdot \alpha_{3} \cdot \alpha_{4}$.

Proof. Since the generalized Kummer variety is the fibre over a point, its class must be the pullback of $x \in H^{4}(A)$ under $\Sigma$. But $\Sigma^{*}(x)=\Sigma^{*}\left(a_{1}\right) \cdot \Sigma^{*}\left(a_{2}\right) \cdot \Sigma^{*}\left(a_{3}\right) \cdot \Sigma^{*}\left(a_{4}\right)$, so we have to verify that $\Sigma^{*}\left(a_{i}\right)=\alpha_{i}$. To do this, we want to use the decomposition $\Sigma=\sigma \varrho$. The pullback along $\sigma$ of a class $a \in H^{1}(A, \mathbb{Q})$ on $H^{1}\left(\operatorname{Sym}^{n}(A), \mathbb{Q}\right)$ is given by $a \otimes 1 \otimes \cdots \otimes 1+\cdots+1 \otimes \cdots \otimes 1 \otimes a$. It follows from $(3.9)$ that $\Sigma^{*}\left(a_{i}\right)=(1 /(n-1) !) \mathfrak{q}_{1}(1)^{n-1} \mathfrak{q}_{1}\left(a_{i}\right)|0\rangle$.

The morphism $\theta$ induces a homomorphism of graded rings

$$
\theta^{*}: H^{*}\left(A^{[n]}\right) \longrightarrow H^{*}\left(K_{n-1}(A)\right),
$$

and by the projection formula, we have

$$
\theta_{*} \theta^{*}(\alpha)=\left[K_{n-1}(A)\right] \cdot \alpha .
$$

Lemma 5.4. Let $\beta \in H^{*}\left(K_{n-1}(A), \mathbb{Q}\right)$. Then there is a class $B \in H^{*}\left(A^{[n]}, \mathbb{Q}\right)$ such that $\theta_{*}(\beta)=$ $n^{-4} B \cdot\left[K_{n-1}(A)\right]$.

Proof. For a point $a \in A$, we denote by $t_{a}$ the morphism on $A^{[n]}$ induced by the translation by $a$. Then we consider the morphism $\Theta: K_{n-1}(A) \times A \longrightarrow A^{[n]}$ defined by $\Theta(\xi, a)=t_{a}(\theta(\xi))$. It fits in a pullback diagram

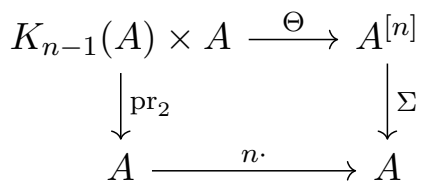

that realizes $K_{n-1}(A) \times A$ as an $n^{4}$-fold covering of $A^{[n]}$ over $A$. Now, for $\beta \in H^{*}\left(K_{n-1}(A), \mathbb{Q}\right)$, set $B:=\Theta_{*}(\beta \otimes 1)$. Then the projection formula gives

$$
\begin{aligned}
B \cdot\left[K_{n-1}(A)\right] & =\Theta_{*}\left(\beta \otimes 1 \cdot \Theta^{*}\left[K_{n-1}(A)\right]\right) \\
& =n^{4} \Theta_{*}((\beta \otimes 1) \cdot(1 \otimes x))=n^{4} \Theta_{*}(\beta \otimes x)=n^{4} \theta_{*}(\beta) .
\end{aligned}
$$

Proposition 5.5. The kernel of $\theta^{*}$ is equal to the annihilator of $\left[K_{n-1}(A)\right]$. 


\section{S. Kapfer And G. Menet}

Proof. Assume $\alpha \in \operatorname{Ker}\left(\theta^{*}\right)$. Then we have $\left[K_{n-1}(A)\right] \cdot \alpha=\theta_{*} \theta^{*}(\alpha)=0$. Conversely, if $\alpha \notin$ $\operatorname{Ker}\left(\theta^{*}\right)$, let $\beta \in H^{*}\left(K_{n-1}(A), \mathbb{Q}\right)$ be the Poincaré dual of $\theta^{*}(\alpha)$, so $\beta \cdot \theta^{*}(\alpha) \neq 0$. Then by the projection formula, $\theta_{*}(\beta) \cdot \alpha \neq 0$. By Lemma 5.4 , there exists $B \in H^{*}\left(A^{[n]}, \mathbb{Q}\right)$ such that $B \cdot\left[K_{n-1}(A)\right] \cdot \alpha \neq 0$. It follows that $\left[K_{n-1}(A)\right] \cdot \alpha \neq 0$.

Corollary 5.6. We have $\theta^{*}(\alpha)=\theta^{*}(\beta)$ if and only if $\left[K_{n-1}(A)\right] \cdot \alpha=\left[K_{n-1}(A)\right] \cdot \beta$.

Proposition 5.7. The annihilator of $\left[K_{n-1}(A)\right]$ in $H^{*}\left(A^{[n]}, \mathbb{Q}\right)$ is the ideal generated by $H^{1}\left(A^{[n]}\right)$.

First we need to recall some material on super algebras (see for instance [DM99]).

DeFinition 5.8. Let $V=V^{+} \oplus V^{-}$be a super vector space and $n \geqslant 0$. Then the supersymmetric power $\operatorname{Sym}^{n}(V)$ of $V$ is a super vector space, given by

$$
\begin{aligned}
& \operatorname{Sym}^{n}(V)=\bigoplus_{p+q=n} \operatorname{Sym}^{p}\left(V^{+}\right) \otimes \Lambda^{q}\left(V^{-}\right), \\
& \operatorname{Sym}^{n}(V)^{+}=\bigoplus_{\substack{p+q=n \\
q \text { even }}} \operatorname{Sym}^{p}\left(V^{+}\right) \otimes \Lambda^{q}\left(V^{-}\right), \quad \operatorname{Sym}^{n}(V)^{-}=\bigoplus_{\substack{p+q=n \\
q \text { odd }}} \operatorname{Sym}^{p}\left(V^{+}\right) \otimes \Lambda^{q}\left(V^{-}\right) .
\end{aligned}
$$

Remark 5.9. The supersymmetric power $\operatorname{Sym}^{n}(V)$ can be realized as a quotient of $V^{\otimes n}$ by an action of the symmetric group $\mathfrak{S}_{n}$. This action can be described as follows: If $\tau \in \mathfrak{S}_{n}$ is a transposition that exchanges two numbers $i<j$, then $\tau$ permutes the corresponding tensor factors in $v_{1} \otimes \cdots \otimes v_{n}$, introducing a sign $(-1)^{\left|v_{i}\right|\left|v_{j}\right|+\left(\left|v_{i}\right|+\left|v_{j}\right|\right) \sum_{i<k<j}\left|v_{k}\right|}$.

Now, let $U$ be a vector space over $\mathbb{Q}$, and look at the exterior algebra $H:=\Lambda^{*} U$. Since $H$ is a super vector space, we can construct the supersymmetric power $\operatorname{Sym}^{n}(H)$. We may identify $\operatorname{Sym}^{n}(H)$ with the space of $\mathfrak{S}_{n}$-invariants in $H^{\otimes n}$ by means of the linear projection operator

$$
\text { pr: } H^{\otimes n} \longrightarrow \operatorname{Sym}^{n}(H), \quad \text { pr }=\frac{1}{n !} \sum_{\pi \in \mathfrak{S}_{n}} \pi .
$$

The multiplication in $H^{\otimes n}$ induces a multiplication on the subspace of invariants, which makes $\operatorname{Sym}^{n}(H)$ a supercommutative algebra.

Since $H$ is generated as an algebra by $U=\Lambda^{1}(U) \subset H$, we may define a homomorphism of algebras

$$
s: H \longrightarrow \operatorname{Sym}^{n}(H), \quad s(u)=\operatorname{pr}(u \otimes 1 \otimes \cdots \otimes 1) \text { for } u \in U,
$$

so $\operatorname{Sym}^{n}(H)$ becomes an algebra over $H$.

Lemma 5.10. The morphism $s$ turns $\operatorname{Sym}^{n}(H)$ into a free module over $H$ for $n \geqslant 1$.

Proof. We start with the tensor power $H^{\otimes n}$ and the ring homomorphism

$$
\iota: H \longrightarrow H^{\otimes n}, \quad h \longmapsto h \otimes 1 \otimes \cdots \otimes 1
$$

that makes $H^{\otimes n}$ into a free $H$-module. Note that $\operatorname{pr} \iota \neq s$ since pr is not a ring homomorphism. (For example, $\operatorname{pr}(\iota(h)) \neq s(h)$ for any non-zero $h \in \Lambda^{2}(U)$.) We therefore modify the $H$-module structure of $H^{\otimes n}$. For $u \in U$, set $u^{(i)}:=1^{\otimes i-1} \otimes u \otimes 1^{\otimes n-i+1} \in H^{\otimes n}$. Then $H^{\otimes n}$ is generated as a $k$-algebra by the elements $\left\{u^{(i)}, u \in U\right\}$. Now, consider the ring automorphism

$$
\sigma: H^{\otimes n} \longrightarrow H^{\otimes n}, \quad u^{(1)} \longmapsto u^{(1)}+u^{(2)}+\cdots+u^{(n)}, \quad u^{(i)} \longmapsto u^{(i)} \text { for } i>1 .
$$

Then we have $\sigma \iota=s$ on $\operatorname{Sym}^{n}(H)$. On the other hand, if $\left\{b_{i}\right\}$ is a $k$-basis of $V$, then $\left\{b_{i}^{(j)}, j>1\right\}$ is a $\iota$-basis for $H^{\otimes n}$ and $\left\{\sigma\left(b_{i}^{(j)}\right)\right\}$ is a $\sigma \iota$-basis for $H^{\otimes n}$. Now, if we project the basis elements, 


\section{INTEGRAL COHOMOLOGY OF THE GENERALIZED KUMMER FOURFOLD}

we get a set $\left\{\operatorname{pr}\left(\sigma\left(b_{i}^{(j)}\right)\right)\right\}$ that spans $\operatorname{Sym}^{n}(H)$. Eliminating linearly dependent vectors (this is possible over the rationals), we get an $s$-basis of $\operatorname{Sym}^{n}(H)$.

Proof of Proposition 5.7. Set $H=H^{*}(A, \mathbb{Q}) \cong \Lambda^{*}\left(H^{1}(A, \mathbb{Q})\right)$, and consider the exact sequence of $H$-modules

$$
0 \longrightarrow J \longrightarrow H \stackrel{x \cdot}{\longrightarrow} H \text {. }
$$

It is clear that $J$ is the ideal in $H$ generated by $H^{1}(A, \mathbb{Q})$. Now, let $J^{(n)}$ be the ideal generated by $H^{1}\left(\operatorname{Sym}^{n}(A), \mathbb{Q}\right)$ in $H^{*}\left(\operatorname{Sym}^{n}(A), \mathbb{Q}\right) \cong \mathbb{S y m}^{n}(H)$. By the freeness result of Lemma 5.10, tensoring with $\operatorname{Sym}^{n}(H)$ yields another exact sequence of $H$-modules,

$$
0 \longrightarrow J^{(n)} \longrightarrow \operatorname{Sym}^{n}(H) \stackrel{\sigma(x) \cdot}{\longrightarrow} \operatorname{Sym}^{n}(H) .
$$

Now, let $\mathfrak{H}$ be the operator algebra spanned by products of $\mathfrak{d}$ and $\mathfrak{q}_{1}(a)$ for $a \in H^{*}(A)$. Let $\mathfrak{C}$ be the graded commutative subalgebra of $\mathfrak{H}$ generated by $\mathfrak{q}_{1}(a)$ for $a \in H^{*}(A)$. The action of $\mathfrak{H}$ on $|0\rangle$ gives $\mathbb{H}$, and the action of $\mathfrak{C}$ on $|0\rangle$ gives $\varrho^{*}\left(H^{*}\left(\operatorname{Sym}^{n}(A), \mathbb{Q}\right)\right) \cong \operatorname{Sym}^{n}(H)$. By sending $\mathfrak{d}$ to the identity, we define a linear map $c: \mathfrak{H} \rightarrow \mathfrak{C}$. Let $J^{[n]}$ be the ideal generated by $H^{1}\left(A^{[n]}, \mathbb{Q}\right)$ in $H^{*}\left(A^{[n]}, \mathbb{Q}\right)$. We claim that for every $\mathfrak{y} \in \mathfrak{H}$,

$$
\mathfrak{y}|0\rangle \in J^{[n]} \Leftrightarrow c(\mathfrak{y})|0\rangle \in J^{[n]} .
$$

To see this, we remark that $H^{1}\left(A^{[n]}, \mathbb{Q}\right) \cong H^{1}(A, \mathbb{Q})$ and that the multiplication with a class in $H^{1}\left(A^{[n]}, \mathbb{Q}\right)$ is given by the operator $\mathfrak{G}_{0}(a)$ for some $a \in H^{1}(A, \mathbb{Q})$. Due to the fact that $\mathfrak{d}$ is also a multiplication operator (of degree 2 ), $\mathfrak{G}_{0}(a)$ commutes with $\mathfrak{d}$. It follows that for $\mathfrak{y}=\mathfrak{G}_{0}(a) \mathfrak{r}$, we have $c(\mathfrak{y})=\mathfrak{G}_{0}(a) c(\mathfrak{r})$.

Now, let $\mathfrak{k}$ be the multiplication operator with the class $\left[K_{n-1}(A)\right]$. We have $[\mathfrak{k}, \mathfrak{d}]=0$. Let $y \in H^{*}\left(A^{[n]}, \mathbb{Q}\right)$ be a class in the annihilator of $\left[K_{n-1}(A)\right]$. We can write $y=\mathfrak{y}|0\rangle$ for a $\mathfrak{y} \in \mathfrak{H}$. Choose $\tilde{y} \in \operatorname{Sym}^{n}(H)$ in such a way that $\varrho^{*}(\tilde{y})=c(\mathfrak{y})|0\rangle$. Then we have

$$
0=\left[K_{n-1}(A)\right] \cdot y=\mathfrak{k} \mathfrak{y}|0\rangle=\mathfrak{k} c(\mathfrak{y})|0\rangle=\varrho^{*}\left(\sigma^{*}(x) \cdot \tilde{y}\right) .
$$

Since $\varrho^{*}$ is injective, $\tilde{y}$ is in the annihilator of $\sigma^{*}(x)$, so $\tilde{y} \in J^{(n)}$. It follows that $c(\mathfrak{y})|0\rangle$ and $y$ are in the ideal generated by $H^{1}\left(A^{[n]}, \mathbb{Q}\right)$.

Theorem 5.11 ([Bea83, Théorème 4]). The variety $K_{n-1}(A)$ is an irreducible holomorphically symplectic manifold. In particular, it is simply connected and the canonical bundle is trivial.

This implies that $H^{2}\left(K_{n-1}(A), \mathbb{Z}\right)$ admits an integer-valued non-degenerate symmetric bilinear form (the Beauville-Bogomolov form) $B_{K_{n-1}(A)}$ which gives $H^{2}\left(K_{n-1}(A), \mathbb{Z}\right)$ the structure of a lattice. Looking, for instance, in the useful table from the introduction of [Rap08], we know that this lattice is isomorphic to $U^{\oplus 3} \oplus\langle-2 n\rangle$ for $n \geqslant 3$. We have the Fujiki formula for $\alpha \in H^{2}\left(K_{n-1}(A), \mathbb{Z}\right)$ :

$$
\int_{K_{n-1}(A)} \alpha^{2 n-2}=n \cdot(2 n-3) ! ! \cdot B_{K_{n-1}(A)}(\alpha, \alpha)^{n-1} .
$$

Proposition 5.12. Assume $n \geqslant 3$. Then $\theta^{*}$ is surjective on $H^{2}\left(A^{[n]}, \mathbb{Z}\right)$.

Proof. By [Bea83, Section 7], the morphism $\theta^{*}: H^{2}\left(A^{[n]}, \mathbb{C}\right) \rightarrow H^{2}\left(K_{n-1}(A), \mathbb{C}\right)$ is surjective. But by [Bri03, Proposition 1], the lattice structure of $\operatorname{Im} \theta^{*}$ is the same as that of $H^{2}\left(K_{n-1}(A)\right)$, so the image of $H^{2}\left(A^{[n]}, \mathbb{Z}\right)$ must be primitive. The result follows.

Notation 5.13. We have seen that for $n \geqslant 3$,

$$
H^{2}\left(K_{n-1}(A), \mathbb{Z}\right) \cong H^{2}(A, \mathbb{Z}) \oplus\left\langle\theta^{*}(\delta)\right\rangle .
$$


We denote the injection $H^{2}(A, \mathbb{Z}) \rightarrow H^{2}\left(K_{n-1}(A), \mathbb{Z}\right)$ by $j$. It can be described by

$$
j: a \longmapsto \frac{1}{(n-1) !} \theta^{*}\left(\mathfrak{q}_{1}(a) \mathfrak{q}_{1}(1)^{n-1}|0\rangle\right) .
$$

Further, we set $e:=\theta^{*}(\delta)$. Using Notation 2.2, we give the following names for classes in $H^{2}\left(K_{n-1}(A), \mathbb{Z}\right)$ :

$$
\begin{array}{lll}
u_{1}:=j\left(a_{1} a_{2}\right), & v_{1}:=j\left(a_{1} a_{3}\right), & w_{1}:=j\left(a_{1} a_{4}\right), \\
u_{2}:=j\left(a_{3} a_{4}\right), & v_{2}:=j\left(a_{4} a_{2}\right), & w_{2}:=j\left(a_{2} a_{3}\right) .
\end{array}
$$

These elements form a basis of $H^{2}\left(K_{n-1}(A), \mathbb{Z}\right)$ with the following intersection relations under the Beauville-Bogomolov form:

$$
B\left(u_{1}, u_{2}\right)=1, \quad B\left(v_{1}, v_{2}\right)=1, \quad B\left(w_{1}, w_{2}\right)=1, \quad B(e, e)=-2 n,
$$

and all other pairs of basis elements are orthogonal.

If $A=E_{1} \times E_{2}$ is the product of two elliptic curves, we choose the $a_{i}$ in such a way that $\left\{a_{1}, a_{2}\right\}$ and $\left\{a_{3}, a_{4}\right\}$ give bases of $H^{1}\left(E_{1}, \mathbb{Z}\right)$ and $H^{1}\left(E_{2}, \mathbb{Z}\right)$, respectively, in the decomposition $H^{1}(A)=H^{1}\left(E_{1}\right) \oplus H^{1}\left(E_{2}\right)$.

\section{Integral cohomology of the generalized Kummer fourfold}

Now, we come to the special case $n=3$, so we study $K_{2}(A)$, the generalized Kummer fourfolds.

Proposition 6.1. The Betti numbers of $K_{2}(A)$ are 1, 0, 7, 8, 108, 8, 7, 0, 1 .

Proof. This follows from Göttsche's formula [Göt94, Section 2.4, p. 49].

First we deduce a description of the integral odd cohomology groups of $K_{2}(A)$ from Proposition 4.6 and Section 5. The middle cohomology $H^{4}\left(K_{2}(A), \mathbb{Z}\right)$ has been studied by Hassett and Tschinkel in [HT13]. We recall some of their results in Section 6.4; then, we proceed by using $\theta^{*}$ to give a partial description of $H^{4}\left(K_{2}(A), \mathbb{Z}\right)$ in terms of the well-understood cohomology of $A^{[3]}$ in Section 6.5. Finally, in Section 6.6, we find a basis of $H^{4}\left(K_{2}(A), \mathbb{Z}\right)$ using the action of the image of the monodromy representation. In order to use the monodromy representation, we start by recalling some notions of monodromy on abelian surfaces in Section 6.2. We will also need some technical calculations related to the action of the symplectic group over finite fields (Section 6.3).

In all the section, we use Notation 2.2 and Notation 5.13.

\subsection{Odd cohomology of the generalized Kummer fourfold}

By means of the morphism $\theta^{*}$, we may express part of the cohomology of $K_{2}(A)$ in terms of Hilbert scheme cohomology. We have seen in Proposition 5.12 that $\theta^{*}$ is surjective in degree 2 and (by duality) also in degree 6 . The next proposition shows that this also holds true in odd degrees.

Proposition 6.2. $A$ basis of $H^{3}\left(K_{2}(A), \mathbb{Z}\right)$ is given by

$$
\begin{aligned}
& \frac{1}{2} \theta^{*}\left(\mathfrak{q}_{1}\left(a_{i}^{*}\right) \mathfrak{q}_{1}(1)^{2}|0\rangle\right), \\
& \frac{1}{2} \theta^{*}\left(\mathfrak{q}_{2}\left(a_{i}\right) \mathfrak{q}_{1}(1)|0\rangle\right),
\end{aligned}
$$




\section{INTEGRAL COHOMOLOGY OF THE GENERALIZED KUMMER FOURFOLD}

and a dual basis of $H^{5}\left(K_{2}(A), \mathbb{Z}\right)$ is given by

$$
\begin{aligned}
& \theta^{*}\left(\mathfrak{q}_{1}\left(a_{i} a_{j}\right) \mathfrak{q}_{1}\left(a_{j}^{*}\right) \mathfrak{q}_{1}(1)|0\rangle\right) \quad \text { for any } j \neq i, \\
& \theta^{*}\left(\mathfrak{q}_{2}\left(a_{i}^{*}\right) \mathfrak{q}_{1}(1)|0\rangle\right) .
\end{aligned}
$$

Proof. The classes (6.1) are Poincaré dual to (6.3) and the classes (6.2) are Poincaré dual to (6.4) by direct computation

$$
\begin{gathered}
\begin{aligned}
\frac{1}{2} \theta^{*}\left(\mathfrak{q}_{1}\left(a_{i}^{*}\right) \mathfrak{q}_{1}(1)^{2}|0\rangle\right) \cdot & \theta^{*}\left(\mathfrak{q}_{1}\left(a_{i} a_{j}\right) \mathfrak{q}_{1}\left(a_{j}^{*}\right) \mathfrak{q}_{1}(1)|0\rangle\right)=\frac{1}{2} \theta^{*}\left(\mathfrak{G}_{0}\left(a_{i}^{*}\right) \mathfrak{q}_{1}\left(a_{i} a_{j}\right) \mathfrak{q}_{1}\left(a_{j}^{*}\right) \mathfrak{q}_{1}(1)|0\rangle\right) \\
& =\frac{1}{2}\left[K_{2}(A)\right] \cdot \mathfrak{q}_{1}\left(a_{i} a_{j}\right) \mathfrak{q}_{1}\left(a_{j}^{*}\right) \mathfrak{q}_{1}\left(a_{i}^{*}\right)=1 \\
\frac{1}{2} \theta^{*}\left(\mathfrak{q}_{2}\left(a_{i}\right) \mathfrak{q}_{1}(1)|0\rangle\right) \cdot & \theta^{*}\left(\mathfrak{q}_{2}\left(a_{i}^{*}\right) \mathfrak{q}_{1}(1)|0\rangle\right)=\theta^{*}\left(\mathfrak{G}_{1}\left(a_{i}\right) \mathfrak{q}_{2}\left(a_{i}^{*}\right) \mathfrak{q}_{1}(1)|0\rangle\right) \\
= & {\left[K_{2}(A)\right] \cdot\left(2 \mathfrak{q}_{3}(x)-\mathfrak{q}_{1}(x)^{2} \mathfrak{q}_{1}(1)\right)|0\rangle=0-1=-1 }
\end{aligned}
\end{gathered}
$$

It remains to show that all classes are integral. It is clear from Lemma 4.1 that (6.1) is integral, while the integrality of $(6.3)$ and (6.4) is obvious. By Proposition 4.6, the class $\frac{1}{2} \mathfrak{q}_{2}\left(a_{i}\right)|0\rangle$ is integral as well. If the operator $\mathfrak{q}_{1}(1)$ is applied, we again get an integral class.

Corollary 6.3. Let $A$ be an abelian surface, and let $g$ be an automorphism of $A$. Let $g^{[[3]]}$ be the automorphism induced by $g$ on $K_{2}(A)$. By Proposition 6.2 , we have $H^{3}\left(K_{2}(A), \mathbb{Z}\right) \cong$ $H^{1}(A, \mathbb{Z}) \oplus H^{3}(A, \mathbb{Z})$, and the action of $g^{[3]]}$ on $H^{3}\left(K_{2}(A), \mathbb{Z}\right)$ is given by the action of $g$ on $H^{1}(A, \mathbb{Z}) \oplus H^{3}(A, \mathbb{Z})$.

Proof. By Proposition 6.2, we have an isomorphism

$$
f: H^{1}(A, \mathbb{Z}) \oplus H^{3}(A, \mathbb{Z}) \rightarrow H^{3}\left(K_{2}(A), \mathbb{Z}\right)
$$

given by $f\left(a_{i}\right)=\frac{1}{2} \theta^{*}\left(\mathfrak{q}_{2}\left(a_{i}\right) \mathfrak{q}_{1}(1)|0\rangle\right)$ and $f\left(a_{i}^{*}\right)=\frac{1}{2} \theta^{*}\left(\mathfrak{q}_{1}\left(a_{i}^{*}\right) \mathfrak{q}_{1}(1)^{2}|0\rangle\right)$ for all $i \in\{1, \ldots, 4\}$. We want to prove that $f \circ g^{*}=g^{[[3]] *} \circ f$. To do so, it is enough to show that $f \circ g^{*}\left(a_{i}\right)=g^{[[3]] *} \circ f\left(a_{i}\right)$ and $f \circ g^{*}\left(a_{i}^{*}\right)=g^{[[3]] *} \circ f\left(a_{i}^{*}\right)$ for all $i \in\{1, \ldots, 4\}$. Let $g^{[3]}$ be the morphism induced by $g$ on $A^{[3]}$. By the definition of $g^{[3]]}$,

$$
g^{[[3]]} \circ \theta=\theta \circ g^{[3]} .
$$

Then, by (6.5) and by the definition of $g^{[3]}$,

$$
\begin{aligned}
g^{[[3]] *} \circ f\left(a_{i}\right) & =g^{[3]] *} \circ \theta^{*}\left(\mathfrak{q}_{2}\left(a_{i}\right) \mathfrak{q}_{1}(1)|0\rangle\right) \\
& =\theta^{*} \circ g^{[3] *}\left(\mathfrak{q}_{2}\left(a_{i}\right) \mathfrak{q}_{1}(1)|0\rangle\right)=\theta^{*}\left(\mathfrak{q}_{2}\left(g^{*}\left(a_{i}\right)\right) \mathfrak{q}_{1}(1)|0\rangle\right) \\
& =f \circ g^{*}\left(a_{i}\right) .
\end{aligned}
$$

We prove $f \circ g^{*}\left(a_{i}^{*}\right)=g^{[[3]] *} \circ f\left(a_{i}^{*}\right)$ with the same method.

\subsection{A monodromy representation on abelian surfaces and generalized Kummer four- folds}

Let $A$ be an abelian surface. We recall that a principal polarization of $A$ is a polarization $L$ such that there exists a basis of $H_{1}(A, \mathbb{Z})$ with respect to which the symplectic bilinear form on $H_{1}(A, \mathbb{Z})$ induced by $c_{1}(L)$,

is given by the matrix

$$
\omega_{L}(x, y)=x \cdot c_{1}(L) \cdot y,
$$

$$
\left(\begin{array}{cccc}
0 & 0 & 1 & 0 \\
0 & 0 & 0 & 1 \\
-1 & 0 & 0 & 0 \\
0 & -1 & 0 & 0
\end{array}\right)
$$




\section{S. Kapfer And G. Menet}

We recall the following result.

Proposition 6.4. Let $(A, L)$ be a principally polarized abelian surface. The group $H_{1}(A, \mathbb{Z})$ is endowed with the symplectic form $\omega_{L}$ defined in (6.6). Let $\operatorname{Mon}\left(H_{1}(A, \mathbb{Z})\right)$ be the image of monodromy representations on $H_{1}(A, \mathbb{Z})$. Then $\operatorname{Mon}\left(H_{1}(A, \mathbb{Z})\right) \supset \operatorname{Sp}\left(H_{1}(A, \mathbb{Z})\right)$.

Proof. The inclusion can be seen as follows. Let $\mathcal{M}_{2}$ be the moduli space of curves of genus 2 and $\mathcal{A}_{2}$ the moduli space of principally polarized abelian surfaces. By the Torelli theorem (see for instance [Mil08, Theorem 12.1]), we have an injection $J: \mathcal{M}_{2} \hookrightarrow \mathcal{A}_{2}$ given by taking the Jacobian of the curve endowed with its canonical polarization. Moreover, the moduli spaces $\mathcal{M}_{2}$ and $\mathcal{A}_{2}$ are both of dimension 3 .

Now, if $\mathscr{C}_{2}$ is a curve of genus 2, we have, by [FM12, Theorem 6.4],

$$
\operatorname{Mon}\left(H_{1}\left(\mathscr{C}_{2}, \mathbb{Z}\right)\right) \supset \operatorname{Sp}\left(H_{1}\left(\mathscr{C}_{2}, \mathbb{Z}\right)\right),
$$

where the symplectic form on $H_{1}\left(\mathscr{C}_{2}, \mathbb{Z}\right)$ is given by the cup product. Then the result follows from the fact that the lattices $H_{1}\left(\mathscr{C}_{2}, \mathbb{Z}\right)$ and $H_{1}\left(J\left(\mathscr{C}_{2}\right), \mathbb{Z}\right)$ are isometric.

Remark 6.5. Let $(A, L)$ be a principally polarized abelian surface and $p$ a prime number. The group $H_{1}(A, \mathbb{Z})$ tensored by $\mathbb{F}_{p}$ can be seen as the group $A[p]$ of points of $p$-torsion on $A$, and the form $\omega_{L} \otimes \mathbb{F}_{p}$ provides a symplectic form on $A[p]$. Then $\operatorname{Mon}(A[p])$, the image of the monodromy representation on $A[p]$, contains the group $\operatorname{Sp}(A[p])$.

We are now ready to recall [HT13, Proposition 5.2] on the monodromy of the generalized Kummer fourfold.

Proposition 6.6. Let $A$ be an abelian surface and $K_{2}(A)$ the associated generalized Kummer fourfold. The image of the monodromy representation on $\Pi=\left\langle Z_{\tau} \mid \tau \in A[3]\right\rangle$ contains the semidirect product $\operatorname{Sp}(A[3]) \ltimes A[3]$, which acts as follows:

$$
f \cdot Z_{\tau}=Z_{f(\tau)} \text { and } \tau^{\prime} \cdot Z_{\tau}=Z_{\tau+\tau^{\prime}}
$$

for all $f \in \operatorname{Sp}(A[3])$ and $\tau^{\prime} \in A[3]$.

\subsection{Actions of the symplectic groups over finite fields}

The aim of this subsection is to provide some special computations used in Section 6.6.

Let $V$ be a symplectic vector space of dimension $n \in 2 \mathbb{N}$ over a field $k$ with a non-degenerate symplectic form $\omega: \Lambda^{2} V \rightarrow k$. A line is a 1-dimensional subspace of $V$ through the origin, and a plane is a 2-dimensional subspace of $V$. A plane $P \subset V$ is called isotropic if $\omega(x, y)=0$ for any $x, y \in P$, otherwise non-isotropic. The symplectic group $\operatorname{Sp} V$ is the set of all linear maps $\phi: V \rightarrow V$ with the property $\omega(\phi(x), \phi(y))=\omega(x, y)$ for all $x, y \in V$.

Proposition 6.7. The symplectic group $\operatorname{Sp} V$ acts transitively on the set of non-isotropic planes as well as on the set of isotropic planes.

Proof. Given two planes $P_{1}$ and $P_{2}$, we may choose vectors $v_{1}, v_{2}, w_{1}, w_{2}$ such that $v_{1}, v_{2}$ span $P_{1}$ and $w_{1}, w_{2}$ span $P_{2}$ and $\omega\left(v_{1}, v_{2}\right)=\omega\left(w_{1}, w_{2}\right)$. We complete $\left\{v_{1}, v_{2}\right\}$ as well as $\left\{w_{1}, w_{2}\right\}$ to symplectic bases of $V$. Then define $\phi\left(v_{1}\right)=w_{1}$ and $\phi\left(v_{2}\right)=w_{2}$. It is now easy to see that the definition of $\phi$ can be extended to the remaining basis elements to give a symplectic morphism.

Remark 6.8. The planes in $V$ can be identified with the simple tensors in $\Lambda^{2} V$ up to multiples. Indeed, given a simple tensor $v \wedge w \in \Lambda^{2} V$, the span of $v$ and $w$ yields the corresponding plane. 


\section{INTEGRAL COHOMOLOGY OF THE GENERALIZED KUMMER FOURFOLD}

Conversely, any two spanning vectors $v$ and $w$ of a plane give the same element $v \wedge w$ (up to multiples).

From now on, we assume that $k$ is finite of cardinality $q$.

Remark 6.9. If $k$ is the field with two elements, then the set of planes in $V$ can be identified with the set $\{\{x, y, z\} \mid x, y, z \in V \backslash\{0\}, x+y+z=0\}$. Observe that for such a $\{x, y, z\}$, $\omega(x, y)=\omega(x, z)=\omega(y, z)$ and this value gives the criterion for isotropy.

Proposition 6.10. (i) The number of lines in $V$ is $\frac{q^{n}-1}{q-1}$.

(ii) The number of planes in $V$ is $\frac{\left(q^{n}-1\right)\left(q^{n-1}-1\right)}{\left(q^{2}-1\right)(q-1)}$

(iii) The number of isotropic planes in $V$ is $\frac{\left(q^{n}-1\right)\left(q^{n-2}-1\right)}{\left(q^{2}-1\right)(q-1)}$.

(iv) The number of non-isotropic planes in $V$ is $\frac{q^{n-2}\left(q^{n}-1\right)}{q^{2}-1}$.

Proof. A line $\ell$ in $V$ is determined by a non-zero vector. There are $q^{n}-1$ non-zero vectors in $V$ and $q-1$ non-zero vectors in $\ell$. A plane $P$ is determined by a line $\ell_{1} \subset V$ and a unique second line $\ell_{2} \in V / \ell_{1}$. We have $\left(q^{2}-1\right) /(q-1)$ choices for $\ell_{1}$ in $P$. The number of planes is therefore

$$
\frac{\frac{q^{n}-1}{q-1} \cdot \frac{q^{n-1}-1}{q-1}}{\frac{q^{2}-1}{q-1}}=\frac{\left(q^{n}-1\right)\left(q^{n-1}-1\right)}{\left(q^{2}-1\right)(q-1)} \text {. }
$$

For an isotropic plane, we have to choose the second line from $\ell_{1}^{\perp} / \ell_{1}$. This is a space of dimension $n-2$, whence the formula. The number of non-isotropic planes is the difference of the two previous numbers.

We want to study the free $k$-module $k[V]$ with basis $\left\{X_{i} \mid i \in V\right\}$. It carries a natural $k$ algebra structure given by $X_{i} X_{j}:=X_{i+j}$ with unit $1=X_{0}$. This algebra is local with maximal ideal $\mathfrak{m}$ generated by all elements of the form $\left(X_{i}-1\right)$.

We introduce an action of $\operatorname{Sp}(4, k)$ on $k[V]$ by setting $\phi\left(X_{i}\right)=X_{\phi(i)}$. Furthermore, the underlying additive group of $V$ acts on $k[V]$ by $v\left(X_{i}\right)=X_{i+v}=X_{i} X_{v}$.

Definition 6.11. For a line $\ell \subset V$, define $S_{\ell}:=\sum_{i \in \ell} X_{i}$. For a vector $0 \neq v \in \ell$, set $S_{v}:=S_{\ell}$.

Lemma 6.12. Let $P \subset V$ be a plane and $\ell_{1}, \ell_{2} \subset P$ two different lines spanning $P$. Then we have

$$
S_{\ell_{1}} S_{\ell_{2}}=\sum_{i \in P} X_{i}=\sum_{\ell \subset P} S_{\ell}
$$

Proof. The first equality is clear. For the second equality, observe that every point $i \in P$ is contained in one line if we count modulo $q$.

Definition 6.13. We define two subsets of $k[V]$ :

$$
M:=\left\{\sum_{i \in P} X_{i} \mid P \subset V \text { is a plane }\right\}, \quad N:=\left\{\sum_{i \in P} X_{i} \mid P \subset V \text { is a non-isotropic plane }\right\} .
$$

Let $(M)$ and $(N)$ be the ideals generated by $M$ and $N$, respectively. Further, let $D$ be the linear span of $\{v(b)-b \mid b \in N, v \in V\}$. Then $D$ is in fact an ideal, namely the product of ideals $\mathfrak{m} \cdot(N)$. 


\section{S. KAPfer AND G. Menet}

Proposition 6.14. We have $(M)=(N)$.

Proof. We have to show that $\sum_{i \in P} X_{i}$ is in $(N)$ for an isotropic plane $P$. Let $v, w$ be two spanning vectors of $P$ and $u$ a vector with $\omega(u, v) \neq 0$. Let $P^{\prime}$ be the non-isotropic plane spanned by $u$ and $v$. By Lemma 6.12, we have

$$
S_{u} S_{v} S_{w}=\sum_{\ell \subset P^{\prime}} S_{\ell} S_{w}=\left(S_{v}+\sum_{\lambda \in k} S_{u+\lambda v}\right) S_{w} .
$$

Now, $w$ spans a non-isotropic plane with every line in $P^{\prime}$, except one, namely the line that contains $v$. So it follows that

$$
\sum_{i \in P} X_{i}=S_{v} S_{w}=S_{u} S_{v} S_{w}-\sum_{\lambda \in k} S_{u+\lambda v} S_{w}
$$

and we see that the right-hand side is an element of $(N)$.

For the rest of this section, we assume $\operatorname{dim}_{k} V=4$.

Proposition 6.15. The following table states the dimensions of $(N)$ and $D$ for some $k$ :

\begin{tabular}{c||c|c|}
$k$ & $\operatorname{dim}_{k}(N)$ & $\operatorname{dim}_{k} D$ \\
\hline $\mathbb{F}_{2}$ & 11 & 5 \\
$\mathbb{F}_{3}$ & 50 & 31 \\
$\mathbb{F}_{5}$ & 355 & 270
\end{tabular}

An easy way to get these numbers is to count elements in the respective vector spaces using a computer.

Remark 6.16. We remark that $X:=\sum_{i \in V} X_{i}$ is in $D$. Indeed, let $P, P^{\prime}$ be two non-isotropic planes with $P \cap P^{\prime}=0$. Then $X=\left(\sum_{i \in P} X_{i}\right)\left(\sum_{i \in P^{\prime}} X_{i}\right)$ and both factors are elements of $(N) \subset \mathfrak{m}$, so $X \in \mathfrak{m} \cdot(N)=D$.

Let us consider some special orthogonal sums. Take two vectors $v, w \in V$ with $\omega(v, w)=1$, and set $x:=(v \wedge w)^{2} \in \operatorname{Sym}^{2}\left(\Lambda^{2} V\right)$. Let $P$ be the plane spanned by $v$ and $w$, and set $y:=$ $\sum_{i \in P} X_{i} \in k[V]$. We now consider the action of $\operatorname{Sp} V$ on $\operatorname{Sym}^{2}\left(\Lambda^{2} V\right) \oplus k[V]$. Let $O$ be the vector space spanned by the elements $\phi(x) \oplus \phi(z)$ for $\phi \in \operatorname{Sp} V$ and $z \in(y)$, and $U$ the vector space spanned by the elements $\phi(x)$ for $\phi \in \operatorname{Sp} V$.

Proposition 6.17. We have, by numerical computations,

\begin{tabular}{c||c|c}
$k$ & $\operatorname{dim}_{k} O$ & $\operatorname{dim}_{k} U$ \\
\hline $\mathbb{F}_{2}$ & 11 & 6 \\
$\mathbb{F}_{3}$ & 51 & 20 \\
$\mathbb{F}_{5}$ & 375 & 20
\end{tabular}

We now prove the following lemma, which we will need for a divisibility argument in Section 6.6.

Lemma 6.18. We assume $k=\mathbb{F}_{3}$. Consider the projections $\operatorname{pr}_{1}: \operatorname{Sym}^{2}\left(\Lambda^{2} V\right) \oplus k[V] \rightarrow \operatorname{Sym}^{2}\left(\Lambda^{2} V\right)$ and $\operatorname{pr}_{2}: \operatorname{Sym}^{2}\left(\Lambda^{2} V\right) \oplus k[V] \rightarrow k[V]$. We have

(i) $\operatorname{dim} \operatorname{Ker}_{\operatorname{pr}}{ }_{2 \mid O}=1$,

(ii) $\operatorname{dim} \operatorname{Ker} \operatorname{pr}_{1 \mid O}=31$. 
Proof. We have $\operatorname{pr}_{1}(O)=U$ and $\operatorname{pr}_{2}(O)=(N)$. Using the dimension tables from Propositions 6.17 and 6.18, we get $\operatorname{dim} \operatorname{Ker}_{p_{1 \mid O}}=\operatorname{dim} O-\operatorname{dim} U=31$ and $\operatorname{dim} \operatorname{Ker}_{2 \mid O}=\operatorname{dim} O-$ $\operatorname{dim}(N)=1$.

\subsection{Hassett and Tschinkel's results}

Notation 6.19. For each $\tau \in A$, let $W_{\tau}$ be the Briançon subscheme of $A^{[3]}$ consisting of the elements supported entirely at the point $\tau$. If $\tau \in A[3]$ is a 3 -torsion point, $W_{\tau}$ is actually a subscheme of $K_{2}(A)$. We will also use the symbol $W_{\tau}$ for the corresponding class in $H^{4}\left(K_{2}(A), \mathbb{Z}\right)$. Further, set

$$
W:=\sum_{\tau \in A[3]} W_{\tau}
$$

For $p \in A$, let $Y_{p}$ be the locus of all $\{x, y, p\}$ in $K_{2}(A)$. The corresponding class $Y_{p} \in H^{4}\left(K_{2}(A), \mathbb{Z}\right)$ is independent of the choice of the point $p$. Then set $Z_{\tau}:=Y_{p}-W_{\tau}$, and let $\Pi$ be the lattice generated by all $Z_{\tau}$ for $\tau \in A[3]$.

Proposition 6.20. Let Sym $:=\operatorname{Sym}^{2}\left(H^{2}\left(K_{2}(A), \mathbb{Z}\right)\right) \subset H^{4}\left(K_{2}(A), \mathbb{Z}\right)$ be the span of products of integral classes in degree 2 . Then Sym $+\Pi \subset H^{4}\left(K_{2}(A), \mathbb{Z}\right)$ is a sublattice of full rank.

Proof. This follows from [HT13, Proposition 4.3].

In Section 4 of [HT13], one finds the following formula:

$$
Z_{\tau} \cdot D_{1} \cdot D_{2}=2 \cdot B_{K_{2}(A)}\left(D_{1}, D_{2}\right)
$$

for all $D_{1}, D_{2} \in H^{2}\left(K_{2}(A), \mathbb{Z}\right), \tau \in A[3]$ and the Beauville-Bogomolov form $B_{K_{2}(A)}$ on $K_{2}(A)$.

Definition 6.21. We set $\Pi^{\prime}:=\Pi \cap \mathrm{Sym}^{\perp}$. It follows from (6.7) that $\Pi^{\prime}$ can be described as the span of all classes of the form $Z_{\tau}-Z_{0}$ or alternatively as the set of all $\sum_{\tau} \alpha_{\tau} Z_{\tau}$ such that $\sum_{\tau} \alpha_{\tau}=0$. Note that in [HT13], the symbol $\Pi^{\prime}$ denotes something different.

Remark 6.22. Since $\mathrm{rkSym}=28$ and $\mathrm{rk} \Pi^{\prime}=80$, the lattice $\operatorname{Sym} \oplus \Pi^{\prime} \subset H^{4}\left(K_{2}(A), \mathbb{Z}\right)$ has full rank.

Proposition 6.23. The class $W$ can be written with the help of the square of half the diagonal as

$$
W=\theta^{*}\left(\mathfrak{q}_{3}(1)|0\rangle\right)=9 Y_{p}+e^{2} .
$$

The second Chern class is non-divisible and given by

$$
c_{2}\left(K_{2}(A)\right)=\frac{1}{3} \sum_{\tau \in A[3]} Z_{\tau}=\frac{1}{3}\left(72 Y_{p}-e^{2}\right) .
$$

Proof. In [HT13, Section 4], one finds the equations

$$
W=\frac{3}{8}\left(c_{2}\left(K_{2}(A)\right)+3 e^{2}\right), \quad Y_{p}=\frac{1}{72}\left(3 c_{2}\left(K_{2}(A)\right)+e^{2}\right),
$$

from which we deduce (6.8) and the second part of (6.9). The first part of equation (6.9) and the non-divisibility are from [HT13, Proposition 5.1].

\subsection{Properties of $\operatorname{Sym}^{2} H^{2}\left(K_{2}(A), \mathbb{Z}\right)$ in $H^{4}\left(K_{2}(A), \mathbb{Z}\right)$}

Proposition 6.24. The image of $H^{4}\left(A^{[3]}, \mathbb{Q}\right)$ under the morphism $\theta^{*}$ equals $\operatorname{Sym}^{2} H^{2}\left(K_{2}(A), \mathbb{Q}\right)$. 


\section{S. Kapfer And G. Menet}

Proof. We start by giving a set of universal generators of $H^{4}\left(A^{[n]}, \mathbb{Q}\right)$ for $n \geqslant 0$. Theorem 5.30 of [LQW02b] asserts that it is possible to do this in terms of multiplication operators. To enumerate elements of $H^{*}(A, \mathbb{Q})$, we follow Notation 2.2. Basis elements of $H^{2}(A, \mathbb{Q})$ will be denoted by $b_{i}$ for $1 \leqslant i \leqslant 6$. Then our set of generators is given by the following table:

\begin{tabular}{l|c} 
Multiplication operator & Number of classes \\
\hline $\mathfrak{G}_{0}\left(a_{1}\right) \mathfrak{G}_{0}\left(a_{2}\right) \mathfrak{G}_{0}\left(a_{3}\right) \mathfrak{G}_{0}\left(a_{4}\right)$ & 1 \\
$\mathfrak{G}_{0}\left(a_{i}\right) \mathfrak{G}_{0}\left(a_{j}\right) \mathfrak{G}_{0}\left(b_{k}\right)$ for $i<j$ & $\left(\begin{array}{c}4 \\
2\end{array}\right) \cdot 6=36$ \\
$\mathfrak{G}_{0}\left(a_{i}\right) \mathfrak{G}_{0}\left(a_{j}^{*}\right)$ & $4 \cdot 4=16$ \\
$\mathfrak{G}_{0}\left(b_{i}\right) \mathfrak{G}_{0}\left(b_{j}\right)$ for $i \leqslant j$ & $\left(\begin{array}{c}6+1 \\
2\end{array}\right)=21$ \\
$\mathfrak{G}_{0}(x)$ & 1 \\
\hline $\mathfrak{G}_{0}\left(a_{i}\right) \mathfrak{G}_{0}\left(a_{j}\right) \mathfrak{G}_{1}(1)$ for $i<j$ & $\left(\begin{array}{l}4 \\
2\end{array}\right)=6$ \\
$\mathfrak{G}_{0}\left(a_{i}\right) \mathfrak{G}_{1}\left(a_{j}\right)$ & $4 \cdot 4=16$ \\
$\mathfrak{G}_{0}\left(b_{i}\right) \mathfrak{G}_{1}(1)$ & 6 \\
$\mathfrak{G}_{1}\left(b_{i}\right)$ & 6 \\
$\mathfrak{G}_{1}(1)^{2}$ & 1 \\
\hline $\mathfrak{G}_{2}(1)$ & 1
\end{tabular}

Any multiplication operator of degree 4 can be written as a linear combination of these 111 classes. Likewise, the dimension of $H^{4}\left(A^{[n]}, \mathbb{Q}\right)$ is 111 for all $n \geqslant 4$, according to Göttsche's formula [Göt94, Section 2.3, p. 35]. However, for smaller $n$, there must be relations of linear dependence. For $n=3$, the 8 classes $\mathfrak{G}_{0}(x), \mathfrak{G}_{1}\left(b_{i}\right)$ and $\mathfrak{G}_{2}(1)$ can be expressed as linear combinations of the others, so we are left with 103 linearly independent classes that form a basis of $H^{4}\left(A^{[3]}, \mathbb{Q}\right)$. Multiplication with the class $\left[K_{2}(A)\right]$ is given by the operator $\mathfrak{G}_{0}\left(a_{1}\right) \mathfrak{G}_{0}\left(a_{2}\right) \mathfrak{G}_{0}\left(a_{3}\right) \mathfrak{G}_{0}\left(a_{4}\right)$ and annihilates every class that contains an operator of the form $\mathfrak{G}_{0}\left(a_{i}\right)$. There are 75 such classes, so by Proposition 5.5, the kernel $\operatorname{Ker} \theta^{*} \subset H^{4}\left(A^{[3]}, \mathbb{Q}\right)$ has dimension at least 75 and the image $\operatorname{Im} \theta^{*}$ has dimension at most $103-75=28$. However, since the image of $\theta^{*}$ must contain $\operatorname{Sym}^{2} H^{2}\left(K_{2}(A), \mathbb{Q}\right)$, which is 28 -dimensional, equality follows.

Proposition 6.25. We have

$$
c_{2}\left(K_{2}(A)\right)=4 u_{1} u_{2}+4 v_{1} v_{2}+4 w_{1} w_{2}-\frac{1}{3} e^{2} .
$$

In particular, $c_{2}\left(K_{2}(A)\right) \in \operatorname{Sym} \otimes \mathbb{Q}$.

Proof. First, note that the defining diagram (5.1) of the Kummer manifold is the pullback of the inclusion of a point, so the normal bundle of $K_{2}(A)$ in $A^{[3]}$ is trivial. The Chern class of the tangent bundle of $K_{2}(A)$ is therefore given by the pullback from $A^{[3]}$; that is, $c\left(K_{2}(A)\right)=\theta^{*}\left(c\left(A^{[3]}\right)\right)$. Proposition 6.24 now allows us to conclude that $c_{2}\left(K_{2}(A)\right) \in \operatorname{Sym} \otimes \mathbb{Q}$.

To obtain the precise formula, we use a result of Boissière [Boi05, Lemma 3.12] giving a commutation relation for the Chern character multiplication operator on the Hilbert scheme. We get

$$
c_{2}\left(A^{[3]}\right)=3 \mathfrak{q}_{1}(1) \mathfrak{L}_{2}(1)|0\rangle-\frac{1}{3} \mathfrak{q}_{3}(1)|0\rangle=\frac{8}{3} \mathfrak{q}_{1}(1) \mathfrak{L}_{2}(1)|0\rangle-\frac{1}{3} \delta^{2} .
$$

With Corollary 5.6, one now shows that $c_{2}\left(K_{2}(A)\right)$ is given as stated.

Remark 6.26. Proposition 6.25 can also be proven using (6.9) and (6.7). 


\section{INTEGRAL COHOMOLOGY OF THE GENERALIZED KUMMER FOURFOLD}

Corollary 6.27. The intersection Sym $\cap \Pi$ is 1-dimensional and spanned by $3 c_{2}\left(K_{2}(A)\right)$.

Proof. By Proposition 6.25 and (6.9), we have $3 c_{2}\left(K_{2}(A)\right) \in$ Sym $\cap \Pi$. Since the ranks of Sym, $\Pi$ and $H^{4}\left(K_{2}(A), \mathbb{Z}\right)$ are 28,81 and 108 , respectively, the intersection cannot contain more.

Corollary 6.28. $Y_{p}=\frac{1}{6}\left(u_{1} u_{2}+v_{1} v_{2}+w_{1} w_{2}\right)$.

Remark 6.29. Using Nakajima operators, we may write

$$
Y_{p}=\frac{1}{9} \theta^{*}\left(\mathfrak{q}_{1}(1) \mathfrak{L}_{2}(1)|0\rangle\right)=\frac{1}{2} \theta^{*}\left(\mathfrak{q}_{1}(x) \mathfrak{q}_{1}(1)^{2}|0\rangle\right) .
$$

We can now summarize all the divisible classes found in Sym in the following proposition.

Proposition 6.30. Let $\left\{e, u_{1}, u_{2}, v_{1}, v_{2}, w_{1}, w_{2}\right\}$ be the integral basis of $H^{2}\left(K_{2}(A), \mathbb{Z}\right)$ as defined in Notation 5.13. We have the following results:

(i) The class $e^{2}$ is divisible by 3 .

(ii) The class $u_{1} u_{2}+v_{1} v_{2}+w_{1} w_{2}$ is divisible by 6 .

(iii) For $y \in\left\{u_{1}, u_{2}, v_{1}, v_{2}, w_{1}, w_{2}\right\}$, the class $e \cdot y$ is divisible by 3 and $y^{2}-\frac{1}{3} e \cdot y$ is divisible by 2 .

Proof. (i) From Proposition 6.25, we see that $e^{2}$ is divisible by 3 , and by Corollary 6.28, the class $u_{1} u_{2}+v_{1} v_{2}+w_{1} w_{2}$ is divisible by 6 .

(ii) We have $y=\theta^{*}\left(\mathfrak{q}_{1}(a) \mathfrak{q}_{1}(1)^{2}|0\rangle\right)$ for some $a \in H^{2}(A, \mathbb{Z})$. A computation yields

$$
e \cdot y=3 \cdot \theta^{*}\left(\mathfrak{q}_{2}(a) \mathfrak{q}_{1}(1)|0\rangle\right) \text { and } y^{2}=\theta^{*}\left(\mathfrak{q}_{1}(a)^{2} \mathfrak{q}_{1}(1)|0\rangle\right)
$$

so $e \cdot y$ is divisible by 3 . Furthermore, by Corollary 4.3 , the class $\frac{1}{2} \mathfrak{q}_{1}(a)^{2} \mathfrak{q}_{1}(1)|0\rangle-\frac{1}{2} \mathfrak{q}_{2}(a) \mathfrak{q}_{1}(1)|0\rangle$ is contained in $H^{4}\left(A^{[3]}, \mathbb{Z}\right)$, so its pullback $\frac{1}{2} y^{2}-\frac{1}{6} e \cdot y$ is an integral class, too.

\subsection{Integral basis of $H^{4}\left(K_{2}(A), \mathbb{Z}\right)$}

From the intersection properties $Z_{\tau} \cdot Z_{\tau^{\prime}}=1$ for $\tau \neq \tau^{\prime}$ and $Z_{\tau}^{2}=4$ from [HT13, Section 4], we compute

$$
\operatorname{discr} \Pi^{\prime}=3^{84} \text {. }
$$

On the other hand, a formula developed in [Kap17] evaluates

$$
\text { discr Sym }=2^{14} \cdot 3^{38}
$$

so the lattices cannot be primitive. Let $\mathrm{Sym}^{\text {sat }}$ and $\Pi^{\text {sat }}$ be the respective primitive overlattices of Sym and $\Pi^{\prime}$ in $H^{4}\left(K_{2}(A), \mathbb{Z}\right)$. Then $\operatorname{Sym} \oplus \Pi^{\prime}$ is a sublattice of $H^{4}\left(K_{2}(A), \mathbb{Z}\right)$ of index $2^{7} \cdot 3^{61}$, and we claim that $\mathrm{Sym}^{\text {sat }} \oplus \Pi^{\text {sat }}$ has index $3^{22}$. We have already found in Proposition 6.30

- 7 linearly independent classes in Sym divisible by 2,

- 8 linearly independent classes in Sym divisible by 3 .

To obtain a basis of $H^{4}\left(K_{2}(A), \mathbb{Z}\right)$, we are now going to find

- 31 linearly independent classes in $\Pi^{\prime}$ divisible by 3 and

- 20 linearly independent classes in $\mathrm{Sym}^{\text {sat }} \oplus \Pi^{\text {ssat }}$, of which 1 is divisible by $3^{3}$ and 19 are divisible by 3 .

The first thing to note is that $\Pi^{\prime}$ is defined topologically for all deformations of $K_{2}(A)$ and that the primitive overlattice of $\Pi^{\prime}$ is a topological invariant, too. By applying a suitable deformation, we may therefore assume, without loss of generality, that $A$ is the product of two elliptic curves 


\section{S. Kapfer And G. Menet}

$A=E_{1} \times E_{2}$. Here, according to Notation 5.13, $u_{1}:=j\left(a_{1} a_{2}\right)$, where $\left\{a_{1}, a_{2}\right\}$ can be seen as a basis of $H^{1}\left(E_{1}\right)$ (this is necessary to obtain expression (6.15)).

Hassett and Tschinkel provide, in [HT13, Proposition 7.1], the class of a Lagrangian plane (that is, a subvariety that is Lagrangian with respect to the holomorphic 2-form of $K_{2}(A)$ and isomorphic to the projective plane $\left.\mathbb{P}^{2}\right) P \subset K_{2}(A)$; this class can be expressed as follows:

$$
[P]=\frac{1}{216}\left(6 u_{1}-3 e\right)^{2}+\frac{1}{8} c_{2}\left(K_{2}(A)\right)-\frac{1}{3} \sum_{\tau \in \Lambda^{\prime}} Z_{\tau},
$$

where $\Lambda^{\prime}=E_{1}[3] \times 0 \subset A[3]$. Hence, by translating this plane by an element $\tau^{\prime} \in A[3]$, we obtain another plane $P^{\prime}$ whose class can be written as

$$
\left[P^{\prime}\right]=\frac{1}{216}\left(6 u_{1}-3 e\right)^{2}+\frac{1}{8} c_{2}\left(K_{2}(A)\right)-\frac{1}{3} \sum_{\tau \in \Lambda^{\prime}+\tau^{\prime}} Z_{\tau} .
$$

By substracting these two expressions, we obtain a first class divisible by 3 in $\Pi^{\prime}$ :

$$
\frac{1}{3} \sum_{\tau \in \Lambda^{\prime}}\left(Z_{\tau}-Z_{\tau+\tau^{\prime}}\right)
$$

By Proposition 6.6, the image of the monodromy representation on the $Z_{\tau}$ contains the symplectic group $\operatorname{Sp}\left(4, \mathbb{F}_{3}\right)$. We know by Proposition 6.7 that $\operatorname{Sp}\left(4, \mathbb{F}_{3}\right)$ acts transitively on the non-isotropic planes of $A[3]$. Hence, modulo $\Pi^{\prime}$, the orbit by $\operatorname{Sp}\left(4, \mathbb{F}_{3}\right)$ of the classes $(6.16)$ is an $\mathbb{F}_{3^{-}}$ vector space naturally isomorphic to $D$, as introduced in Definition 6.13, so by Proposition 6.15, we get a subspace of $\Pi^{\prime}$ of rank 31 of classes divisible by 3 . We add the thirds of these classes to $\Pi^{\prime}$, and we get an overlattice $\Pi^{\prime \text { over }}$ of $\Pi^{\prime}$ such that

$$
\frac{\Pi^{\text {over }}}{\Pi^{\prime}}=\left(\frac{\mathbb{Z}}{3 \mathbb{Z}}\right)^{\oplus 31}
$$

This subspace can be determined by a computer. We describe it in Proposition A.1 (we will see later in this section that $\Pi^{\prime \text { over }}=\Pi^{\prime \text { sat }}$ ).

We are now going to find the classes divisible by 3 in $\mathrm{Sym}^{\mathrm{sat}} \oplus \Pi^{\text {sat }}$. The class $Z_{0}$ is contained in neither Sym nor in $\Pi^{\prime}$. It can be written as follows:

$$
Z_{0}=\frac{\sum_{\tau \in A[3]} Z_{\tau}-\sum_{\tau \in A[3]}\left(Z_{\tau}-Z_{0}\right)}{81} \stackrel{(6.9)}{=} \frac{c_{2}\left(K_{2}(A)\right)-\frac{1}{3} \sum_{\tau \in A[3]}\left(Z_{\tau}-Z_{0}\right)}{27}
$$

where $\frac{1}{3} \sum_{\tau \in A[3]}\left(Z_{\tau}-Z_{0}\right)$ can be expressed as a linear combination of the 31 classes of Proposition A.1 by Remark 6.16. Hence, $Z_{0}$ is the class in $\mathrm{Sym}^{\text {sat }} \oplus \Pi^{\prime \text { sat }}$ divisible by 27. Let us now find the remaining 19 classes divisible by 3 .

We rearrange (6.15) using (6.10):

$$
\begin{aligned}
{[P] } & =\frac{1}{216}\left(6 u_{1}-3 e\right)^{2}+\frac{1}{8} c_{2}\left(K_{2}(A)\right)-\frac{1}{3} \sum_{\tau \in \Lambda^{\prime}} Z_{\tau} \\
& =\frac{36 u_{1}^{2}+9 e^{2}-36 u_{1} \cdot e}{216}+\frac{W}{3}-\frac{3}{8} e^{2}-\frac{1}{3} \sum_{\tau \in \Lambda^{\prime}} Z_{\tau} \\
& =\frac{u_{1}^{2}-2 e^{2}-u_{1} \cdot e}{6}+\frac{W}{3}-\frac{1}{3} \sum_{\tau \in \Lambda^{\prime}} Z_{\tau} .
\end{aligned}
$$

By Proposition 6.30, the classes $e^{2}$ and $u_{1} \cdot e$ are both divisible by 3 , and by (6.10), the class $W$ 


\section{INTEGRAL COHOMOLOGY OF THE GENERALIZED KUMMER FOURFOLD}

is divisible by 3 , so the following class is integral:

$$
\mathfrak{N}:=\frac{u_{1}^{2}+\sum_{\tau \in \Lambda^{\prime}}\left(Z_{\tau}-Z_{0}\right)}{3} .
$$

From the considerations in Section 6.2, we know that the group $\operatorname{Sp}(A[3]) \ltimes A[3] \subset \operatorname{Mon}(\Pi)$ is a natural extension of $\operatorname{Sp}\left(H_{1}(A, \mathbb{Z})\right) \ltimes A[3] \subset \operatorname{Mon}\left(H_{1}(A, \mathbb{Z})\right)$. Isometries of the image of the monodromy representation on $H_{1}(A, \mathbb{Z})$ extend naturally to isometries of the image of the monodromy representation of $H^{4}\left(K_{2}(A), \mathbb{Z}\right)$ acting on $\Pi$ as described in Proposition 6.6 and acting on Sym by commuting with the map $j$ defined in Notation 5.13. Hence, the group $\operatorname{Sp}\left(H_{1}(A, \mathbb{Z})\right) \ltimes A[3]$ can be seen as a subgroup of $\operatorname{Mon}\left(H^{4}\left(K_{2}(A), \mathbb{Z}\right)\right)$.

We will now conclude using this monodromy action of $\operatorname{Sp}\left(H_{1}(A, \mathbb{Z})\right) \ltimes A[3]$ on the element $\mathfrak{N}$ and the considerations from Section 6.3. Proposition 6.17 states that the orbit of $\mathfrak{N}$ under the action of $\operatorname{Sp}(A[3]) \ltimes A[3]$ spans a space of rank 51 modulo Sym $\oplus \Pi^{\prime}$. However, by Lemma 6.18, the intersection of that space with $\mathrm{Sym}^{\text {sat }}$ is 1-dimensional and the intersection with $\Pi^{\text {sat }}$ has dimension 31, so we are left with 19 linearly independent elements which provide 19 elements in $H^{4}\left(K_{2}(A), \mathbb{Z}\right) / \mathrm{Sym}^{\mathrm{sat}} \oplus \Pi^{\text {ssat }}$. These 19 independent classes can be enumerated using a computer; see Proposition A.2.

We will now check that we have found all the classes in $H^{4}\left(K_{2}(A), \mathbb{Z}\right)$. Let Sym ${ }^{\text {over }}$ be the overlattice of Sym obtained by adding all the classes from Proposition 6.30:

$$
\frac{\mathrm{Sym}}{\mathrm{Sym}^{\text {over }}}=(\mathbb{Z} / 2 \mathbb{Z})^{\oplus 7} \oplus(\mathbb{Z} / 3 \mathbb{Z})^{\oplus 8} \text {. }
$$

Let $F$ be the overlattice of $\operatorname{Sym}^{\text {sat }} \oplus \Pi^{\text {ssat }}$ obtained by adding $Z_{0}$ and the thirds of all classes of Proposition A.2:

We have to show that

$$
\frac{F}{\operatorname{Sym}^{\text {sat }} \oplus \Pi^{\prime \text { sat }}}=(\mathbb{Z} / 27 \mathbb{Z}) \oplus(\mathbb{Z} / 3 \mathbb{Z})^{\oplus 19}
$$

$$
\mathrm{Sym}^{\text {over }}=\mathrm{Sym}^{\text {sat }}, \quad \Pi^{\text {over }}=\Pi^{\text {sat }}
$$

and $F=H^{4}\left(K_{2}(A), \mathbb{Z}\right)$. This can be seen by calculating the discriminants. We have Sym ${ }^{\text {over }} \subset$ Sym $^{\text {sat }}$ and $\Pi^{\text {over }} \subset \Pi^{\text {sat }}$; hence, to prove (6.20), we only have to show that discr Sym ${ }^{\text {over }}=$ discr Sym ${ }^{\text {sat }}$ and discr $\Pi^{\prime \text { over }}=$ discr $\Pi^{\text {sat }}$. By (6.14), (6.18) and (2.6), the lattice Sym ${ }^{\text {over }}$ has discriminant $3^{22}$. Moreover, by (6.13), (6.17) and (2.6), the lattice $\Pi^{\prime \text { over }}$ has discriminant $3^{22}$. Therefore,

$$
\operatorname{discr}\left(\operatorname{Sym}^{\text {over }} \oplus \Pi^{\text {over }}\right)=3^{44} \text {. }
$$

It follows that discr $\left(\operatorname{Sym}^{\text {sat }} \oplus \Pi^{\text {sat }}\right)$ divides $3^{44}$. Hence, by (2.6) and (6.19), discr $F$ divides 1 , so, necessarily, discr $F=1$. Therefore, necessarily, $F=H^{4}\left(K_{2}(A), \mathbb{Z}\right)$. Furthermore, we have $\operatorname{discr}\left(\operatorname{Sym}^{\text {sat }} \oplus \Pi^{\text {sat }}\right)=3^{44}$, which shows by $(6.21)$ that $\operatorname{Sym}^{\text {over }}=\operatorname{Sym}^{\text {sat }}$ and $\Pi^{\prime \text { over }}=\Pi^{\text {sat }}$.

We summarize the description of the integral basis of $H^{4}\left(K_{2}(A), \mathbb{Z}\right)$ in the following theorem.

Theorem 6.31. Let $A$ be an abelian variety. We use Notation 5.13 and Notation 6.19.

(i) Let $\operatorname{Sym}^{\text {sat }}$ be the primitive overlattice of $\operatorname{Sym}^{2}\left(H^{2}\left(K_{2}(A), \mathbb{Z}\right)\right)$ in $H^{4}\left(K_{2}(A), \mathbb{Z}\right)$. The group $\operatorname{Sym}^{\text {sat }} / \operatorname{Sym}^{2}\left(H^{2}\left(K_{2}(A), \mathbb{Z}\right)\right)=(\mathbb{Z} / 2 \mathbb{Z})^{\oplus 7} \oplus(\mathbb{Z} / 3 \mathbb{Z})^{\oplus 8}$ is generated by the elements

$$
\frac{e \cdot y}{3}, \frac{y^{2}-\frac{1}{3} e \cdot y}{2} \text { for } y \in\left\{u_{1}, u_{2}, v_{1}, v_{2}, w_{1}, w_{2}\right\}, \frac{e^{2}}{3} \text { and } \frac{u_{1} \cdot u_{2}+v_{1} \cdot v_{2}+w_{1} \cdot w_{2}}{6} \text {. }
$$

(ii) Let $\Pi^{\prime}$ be the lattice from Definition 6.21 , and let $\Pi^{\prime \text { sat }}$ be the primitive overlattice of $\Pi^{\prime}$ in 


\section{S. Kapfer And G. Menet}

$H^{4}\left(K_{2}(A), \mathbb{Z}\right)$. The group $\Pi^{\text {ssat }} / \Pi^{\prime}=(\mathbb{Z} / 3 \mathbb{Z})^{\oplus 31}$ is generated by the classes

$$
\frac{1}{3} \sum_{\tau \in \Lambda}\left(Z_{\tau}-Z_{\tau+\tau^{\prime}}\right)
$$

with $\Lambda$ a non-isotropic group and $\tau^{\prime} \in A[3]$. Moreover, a basis of $\Pi^{\prime s a t} / \Pi^{\prime}$ is provided by the 31 classes described in Proposition A.1.

(iii) We have

$$
\frac{H^{4}\left(K_{2}(A), \mathbb{Z}\right)}{\operatorname{Sym}^{\text {sat }} \oplus \Pi^{\text {sat }}}=\left(\frac{\mathbb{Z}}{27 \mathbb{Z}}\right) \oplus\left(\frac{\mathbb{Z}}{3 \mathbb{Z}}\right)^{\oplus 19} .
$$

Moreover, this group is generated by the class $Z_{0}$ and the 19 classes described in Proposition A.2.

Moreover, since $\mathrm{Sym}^{\text {over }}=\mathrm{Sym}^{\text {sat }}$, from the proofs of Propositions 6.25, 6.30 and Remark 6.29, we obtain the following corollary.

Corollary 6.32. The image of $H^{4}\left(A^{[3]}, \mathbb{Z}\right)$ under $\theta^{*}$ is equal to Sym ${ }^{\text {sat }}$.

\subsection{Conclusion on the morphism to the Hilbert scheme}

Let us summarize our results on $\theta^{*}$.

Theorem 6.33. Let $A$ be an abelian variety and $\left(b_{i}\right) \subset H^{2}(A, \mathbb{Z})$ an integral basis. Let $\theta: K_{2}(A)$ $\hookrightarrow A^{[3]}$ be the embedding. We also use Notation 2.2 .

The homomorphism $\theta^{*}: H^{*}\left(A^{[3]}, \mathbb{Z}\right) \rightarrow H^{*}\left(K_{2}(A), \mathbb{Z}\right)$ of graded rings is surjective in every degree except 4. Moreover, the image of $H^{4}\left(A^{[3]}, \mathbb{Z}\right)$ is the primitive overlattice of the group $\operatorname{Sym}^{2}\left(H^{2}\left(K_{2}(A), \mathbb{Z}\right)\right)$. The kernel of $\theta^{*}$ is the ideal generated by $H^{1}\left(A^{[3]}, \mathbb{Z}\right)$. The images by $\theta^{*}$ of the following integral classes provide a basis of $\operatorname{Im} \theta^{*}$ :

\begin{tabular}{c||l|l} 
Degree & Preimage of class & Alternative name \\
\hline 0 & $\frac{1}{6} \mathfrak{q}_{1}(1)^{3}|0\rangle$ & 1 \\
\hline 2 & $\frac{1}{2} \mathfrak{q}_{1}\left(b_{i}\right) \mathfrak{q}_{1}(1)^{2}|0\rangle$ for $1 \leqslant i \leqslant 6$ & $j\left(b_{i}\right)$ \\
& $\frac{1}{2} \mathfrak{q}_{2}(1) \mathfrak{q}_{1}(1)|0\rangle$ & $e$ \\
\hline 3 & $\frac{1}{2} \mathfrak{q}_{1}\left(a_{i}^{*}\right) \mathfrak{q}_{1}(1)^{2}|0\rangle$ & \\
& $\frac{1}{2} \mathfrak{q}_{2}\left(a_{i}\right) \mathfrak{q}_{1}(1)|0\rangle$ & \\
\hline 4 & $\mathfrak{q}_{1}\left(b_{i}\right) \mathfrak{q}_{1}\left(b_{j}\right) \mathfrak{q}_{1}(1)|0\rangle$ for $1 \leqslant i \leqslant j \leqslant 6$, but $\left(b_{i}, b_{j}\right) \neq\left(a_{1} a_{2}, a_{3} a_{4}\right)$ & \\
& $\frac{1}{2} \mathfrak{q}_{1}(x) \mathfrak{q}_{1}(1)^{2}|0\rangle($ instead of the missing case above $)$ & \\
& $\frac{1}{2}\left(\mathfrak{q}_{1}\left(b_{i}\right)^{2}-\mathfrak{q}_{2}\left(b_{i}\right)\right) \mathfrak{q}_{1}(1)|0\rangle$ & \\
& $\frac{1}{3} \mathfrak{q}_{3}(1)|0\rangle$ & \\
\hline 5 & $\mathfrak{q}_{1}\left(a_{i} a_{j}\right) \mathfrak{q}_{1}\left(a_{j}^{*}\right) \mathfrak{q}_{1}(1)|0\rangle$ for any choice of $j \neq i$ & \\
& $\mathfrak{q}_{2}\left(a_{i}^{*}\right) \mathfrak{q}_{1}(1)|0\rangle$ & \\
\hline 6 & $\mathfrak{q}_{1}\left(a_{i}^{*}\right) \mathfrak{q}_{1}\left(a_{j}^{*}\right) \mathfrak{q}_{1}(1)|0\rangle$ for $1 \leqslant i<j \leqslant 4$ & top class
\end{tabular}




\section{INTEGRAL COHOMOLOGY OF THE GENERALIZED KUMMER FOURFOLD}

Proof. The table is established by the following results: For degree 2, see Proposition 5.12. Since the Poincaré duality pairing on $K_{2}(A)$ can be evaluated using projection formula (5.3), the dual classes of degree 6 are easily computed. The odd degrees are treated by Proposition 6.2. Classes of degree 4 are studied in Sections 6.5 and 6.6. The classes are chosen in such a way that they give a basis of $\mathrm{Sym}^{\text {sat }}$, which is possible by Corollary 6.32 . The condition $\left(b_{i}, b_{j}\right) \neq\left(a_{1} a_{2}, a_{3} a_{4}\right)$ is more or less arbitrary, but we had to remove one class to avoid a relation of linear dependence.

The kernel of $\theta^{*}$ is described by Propositions 5.5 and 5.7.

\section{Symplectic involutions on $K_{2}(A)$}

By Section 6.1 and [MTW18], it is now possible to classify the symplectic involutions on $K_{2}(A)$.

Let $X$ be an irreducible symplectic manifold. Let

$$
\nu: \operatorname{Aut}(X) \rightarrow \operatorname{Aut}\left(H^{2}(X, \mathbb{Z})\right)
$$

be the natural morphism. Hassett and Tschinkel [HT13, Theorem 2.1] have shown that Ker $\nu$ is a deformation invariant. Let $X$ be an irreducible symplectic fourfold of Kummer type. Then Oguiso [Ogu12] showed that $\operatorname{Ker} \nu=(\mathbb{Z} / 3 \mathbb{Z})^{\oplus 3} \rtimes \mathbb{Z} / 2 \mathbb{Z}$.

Let $A$ be an abelian variety and $g$ an automorphism of $A$. Let us denote by $T_{A[3]}$ the group of translations of $A$ by elements of $A[3]$ and by $\operatorname{Aut}_{\mathbb{Z}}(A)$ the group of $\mathbb{Z}$-linear automorphisms of $A$. If $g \in T_{A[3]} \rtimes \operatorname{Aut}_{\mathbb{Z}}(A)$, then $g$ induces a natural automorphism on $K_{2}(A)$. We denote the induced automorphism by $g^{[[3]]}$. If there is no ambiguity, we also denote the induced automorphism by the same letter $g$ to avoid too complicated formulas.

When $X=K_{2}(A)$, the kernel Ker $\nu$ can be described precisely as follows.

Corollary 7.1 ([BNS11, Corollary 3.3]). Let $A$ be a complex torus and $\nu$ : Aut $\left(K_{2}(A)\right) \rightarrow$ $\operatorname{Aut}\left(H^{2}\left(K_{2}(A), \mathbb{Z}\right)\right)$ the natural map. Then $\operatorname{Ker} \nu=T_{A[3]} \rtimes\left(-\operatorname{id}_{A}\right)^{[[3]]}$.

\subsection{The Torelli theorem}

To prove Theorem 7.5(i), we will need to use the global Torelli theorem for irreducible holomorphic symplectic (IHS) manifolds stated by Markman in [Mar11]. We recall this theorem in this section.

Let $X_{1}$ and $X_{2}$ be IHS manifolds. We say that the isomorphism $f: H^{*}\left(X_{1}, \mathbb{Z}\right) \cong H^{*}\left(X_{2}, \mathbb{Z}\right)$ is a parallel-transport operator if there exist a smooth and proper family $\pi: \mathcal{X} \rightarrow B$ of IHS manifolds over an analytic base $B$, points $b_{i} \in B$, isomorphisms $\psi_{i}: X_{i} \rightarrow \mathcal{X}_{b_{i}}$ for $i=1,2$ and a continuous path $\gamma:[0,1] \rightarrow B$ satisfying $\gamma(0)=b_{1}$ and $\gamma(1)=b_{2}$, such that the parallel transport in the local system $R \pi_{*} \mathbb{Z}$ along $\gamma$ induces the homomorphism $\psi_{2 *} \circ f \circ \psi_{1}^{*}: H^{*}\left(\mathcal{X}_{b_{1}}, \mathbb{Z}\right) \stackrel{\cong}{\rightarrow} H^{*}\left(\mathcal{X}_{b_{2}}, \mathbb{Z}\right)$. An isomorphism $g: H^{2}\left(X_{1}, \mathbb{Z}\right) \stackrel{\cong}{\rightarrow} H^{2}\left(X_{2}, \mathbb{Z}\right)$ is said to be a parallel-transport operator if it is the second graded summand of a parallel-transport operator $f$ as above. Note that an automorphism $g: H^{2}(X, \mathbb{Z}) \stackrel{\cong}{\rightarrow} H^{2}(X, \mathbb{Z})$ of the second cohomology group of an IHS manifolds $X$ which is a parallel-transport operator is a monodromy operator. We denote the subgroup of monodromy operators of $X$ by $\operatorname{Mon}^{2}(X) \subset \mathcal{O}\left(H^{2}(X, \mathbb{Z})\right)$.

Theorem 7.2 ([Mar11, Theorem 1.3]). Let $X$ and $Y$ be two IHS manifolds which are deformation equivalent. Then, $X$ and $Y$ are bimeromorphic if and only if there exists a parallel-transport operator $f: H^{2}(X, \mathbb{Z}) \rightarrow H^{2}(Y, \mathbb{Z})$ which is a Hodge isometry.

To use this theorem, it is important to know the group $\operatorname{Mon}^{2}(X)$. In the case of an irre- 


\section{S. Kapfer and G. Menet}

ducible symplectic manifold of Kummer type $X$, the group $\operatorname{Mon}^{2}(X)$ was described by Mongardi in [Mon16]. Let $O^{+}\left(H^{2}(X, \mathbb{Z})\right)$ be the subgroup of $O\left(H^{2}(X, \mathbb{Z})\right)$ that preserve the orientation of the positive cone. Let $\mathcal{W}(X)$ be the subgroup of $O^{+}\left(H^{2}(X, \mathbb{Z})\right)$ acting as \pm 1 on the discriminant group $A_{H^{2}(X, \mathbb{Z})}:=H^{2}(X, \mathbb{Z})^{*} / H^{2}(X, \mathbb{Z})$. Let $\chi$ be the character corresponding to the action on $A_{H^{2}(X, \mathbb{Z})}$. We denote the kernel of deto $\chi: \mathcal{W}(X) \rightarrow\{ \pm 1\}$ by $\mathcal{N}(X)$.

Theorem 7.3 ([Mon16, Theorem 2.3]). Let $X$ be an irreducible symplectic manifold of Kummer type. Then $\operatorname{Mon}^{2}(X)=\mathcal{N}(X)$.

Let $\Lambda_{n}$ be the lattice $U^{\oplus 3} \oplus(-2-2 n)$. Let $X$ be an irreducible symplectic $2 n$-fold of Kummer type; an isometry $\varphi: H^{2}(X, \mathbb{Z}) \rightarrow \Lambda_{n}$ is called a mark, and the pair $(X, \varphi)$ is called a marked irreducible symplectic $2 n$-fold of Kummer type. We denote the moduli space of marked irreducible symplectic $2 n$-folds of Kummer type by $\mathcal{M}_{\Lambda_{n}}$. Moreover, we recall that the period map is defined as follows:

$$
\begin{aligned}
\mathscr{P}: \mathcal{M}_{\Lambda_{n}} & \longrightarrow \Omega_{\Lambda}:=\left\{x \in \mathbb{P}\left(\Lambda_{n} \otimes \mathbb{C}\right) \mid x^{2}=0 \text { and } x \cdot \bar{x}>0\right\}, \\
(X, \varphi) & \longmapsto \varphi\left(H^{0}\left(X, \Omega_{X}^{2}\right)\right) .
\end{aligned}
$$

Corollary 7.4. Let $X$ be an irreducible symplectic $2 n$-fold of Kummer type, with $n+1$ a prime power. Let $A$ be a 2-dimensional complex torus; we denote its dual torus by $A^{*}$. If there exists a Hodge isometry $f: H^{2}(X, \mathbb{Z}) \rightarrow H^{2}\left(K_{n}(A), \mathbb{Z}\right)$, then $X$ is bimeromorphic to $K_{n}(A)$ or to $K_{n}\left(A^{*}\right)$.

Proof. We are going to use Theorem 7.2. To do so, we have to understand when $f$ is a paralleltransport operator. Let $\varphi$ be a mark of $X$; since $X$ is of Kummer type, we can find a mark $\psi$ of $K_{n}(A)$ such that $\left(K_{n}(A), \psi\right)$ and $(X, \varphi)$ are in the same connected component $\mathcal{M}_{\Lambda_{n}}^{o}$ of the moduli space $\mathcal{M}_{\Lambda_{n}}$. In particular, this means that $\psi^{-1} \circ \varphi$ is a parallel-transport operator. We are now going to consider $f \circ \varphi^{-1} \circ \psi \in O\left(H^{2}\left(K_{n}(A), \mathbb{Z}\right)\right)$. We can assume that $f \circ \varphi^{-1} \circ \psi \in$ $O^{+}\left(H^{2}\left(K_{n}(A), \mathbb{Z}\right)\right)$, by changing $f$ to $-f$ if necessary. Moreover, since $n+1$ is a prime power, by [MM17, Lemma 4.3], we can find $\nu \in \mathcal{N}\left(K_{n}(A)\right)=\operatorname{Mon}\left(K_{n}(A)\right)$ such that $f \circ \varphi^{-1} \circ \psi \circ \nu(\delta)=\delta$, where $\delta$ is half the class of the diagonal divisor in $K_{n}(A)$. Hence, we can exchange the mark $\psi$ for the mark $\psi^{\prime}:=\psi \circ \nu$, keeping $\left(K_{n}(A), \psi^{\prime}\right)$ in $\mathcal{M}_{\Lambda_{n}}^{o}$.

As in [MM17, Section 4], we now consider the dual complex torus $A^{*}$ of $A$. Then $H^{1}\left(A^{*}, \mathbb{Z}\right)$ is isomorphic to $H^{1}(A, \mathbb{Z})^{*}$. Let $\bar{\tau}$ be the composition of natural isomorphisms $H^{2}(A, \mathbb{Z}) \simeq$ $H^{2}(A, \mathbb{Z})^{*} \simeq H^{2}\left(A^{*}, \mathbb{Z}\right)$, where the first isomorphism is induced by the intersection pairing. Let $\tau: H^{2}\left(K_{2}(A), \mathbb{Z}\right) \rightarrow H^{2}\left(K_{2}\left(A^{*}\right), \mathbb{Z}\right)$ be the isomorphism restricting to $\delta^{\perp}$ as $-\bar{\tau}$ and mapping the class $\delta$ to half the class of the diagonal divisor in $K_{2}\left(A^{*}\right)$. The isometry $\bar{\tau}$ is also constructed in [Shi78, Lemma 3], and it is shown there that it preserves the period. Moreover, by [MM17, Proposition 4.6], we have either $f \circ \varphi^{-1} \circ \psi^{\prime} \in \mathcal{N}\left(K_{n}(A)\right)=\operatorname{Mon}\left(K_{n}(A)\right)$, or $\tau \circ f \circ \varphi^{-1} \circ \psi^{\prime} \in \mathcal{N}\left(K_{n}(A)\right)=\operatorname{Mon}\left(K_{n}(A)\right)$. So either $f$ or $\tau \circ f$ is a parallel-transport operator. Then, we conclude the proof with Theorem 7.2.

\subsection{Uniqueness and fixed locus}

Theorem 7.5. Let $X$ be an irreducible symplectic fourfold of Kummer type and $\iota$ a symplectic involution on $X$.

(i) We have $\iota \in \operatorname{Ker} \nu$.

(ii) Let $A$ be an abelian surface. Then the pair $(X, \iota)$ is deformation equivalent to $\left(K_{2}(A), t_{\tau}\right.$ 。 $\left.\left(-\mathrm{id}_{A}\right)^{[[3]]}\right)$, where $t_{\tau}$ is the morphism induced on $K_{2}(A)$ by the translation by $\tau \in A[3]$. 


\section{INTEGRAL COHOMOLOGY OF THE GENERALIZED KUMMER FOURFOLD}

(iii) The fixed locus of $\iota$ is given by a K3 surface and 36 isolated points.

Proof of part (i). If $\iota \notin \operatorname{Ker} \nu$, by the classification of [MTW18, Section 5], the unique possible action of $\iota$ on $H^{2}(X, \mathbb{Z})$ is given by $H^{2}(X, \mathbb{Z})^{\iota}=U \oplus(2)^{\oplus 2} \oplus(-6)$. We will show that this is impossible. Let us assume that $H^{2}(X, \mathbb{Z})^{\iota}=U \oplus(2)^{\oplus 2} \oplus(-6)$; we will find a contradiction. Denote the sublattice $U \oplus(2)^{\oplus 2} \oplus(-6)$ of $\Lambda_{2}$ by $\Lambda_{\iota}$. The proof is organized as follows. First we will show that $(X, \iota)$ deforms to a pair $\left(K_{2}(A), i\right)$, where $i$ is a natural involution (that is, an involution induced by an involution on $A$ ). Then we will see that this is impossible, using [MTW15, Section 4] and Corollary 6.3.

As explained after [Mon13, Remark 2], we can find a pair $\left(Y, \iota^{\prime}\right)$ which is deformation equivalent to $(X, \iota)$ such that there exist two marks $\varphi, \varphi^{\prime}$ and a generic complex torus $A$ with

$$
\mathscr{P}(Y, \varphi)=\mathscr{P}\left(K_{2}(A), \varphi^{\prime}\right) .
$$

We recall in a few words how this is shown in [Mon13]. First, since $\iota$ is symplectic, a period of $X$ is contained in the sub-period domain $\Omega_{\Lambda_{\iota}}:=\left\{x \in \mathbb{P}\left(\Lambda_{\iota} \otimes \mathbb{C}\right) \mid x^{2}=0\right.$ and $\left.x \cdot \bar{x}>0\right\}$. Moreover, we can find an $x \in \Omega_{\Lambda_{\iota}}$ such that $x^{\perp} \cap \Lambda_{\iota}=\mathbb{Z} d$ with $d^{2}=-6$ and $d \cdot \Lambda_{2}=6 \mathbb{Z}$. Furthermore, we can link $x$ to a period of $X$ by a chain of twisted lines in $\Omega_{\Lambda_{\iota}}$ (see for instance [Huy12, Proposition 3.7]). Then [Mon13, Remark 1] explains that there exist a pair $\left(Y, \iota^{\prime}\right)$, deformation equivalent to $(X, \iota)$, and a mark $\varphi$ such that $\mathscr{P}(Y, \varphi)=x$. Moreover, $d^{\perp}$ in $\Lambda_{2}$ is isomorphic to the torus lattice, so by the surjectivity of the period map of a 2-dimensional torus, we can find a torus $A$ and a mark $\varphi^{\prime}$ such that $\mathscr{P}\left(K_{2}(A), \varphi^{\prime}\right)=x$. Observe, in addition, that we have chosen $x$ such that the $\operatorname{NS}(A)$ is minimal, that is, $\operatorname{NS}(A) \simeq \mathcal{S}_{\iota^{\prime}}:=\left(H^{2}(Y, \mathbb{Z})^{\iota^{\prime}}\right)^{\perp}$. To be more precise, if $j: H^{2}\left(K_{2}(A), \mathbb{Z}\right) \rightarrow H^{2}(A, \mathbb{Z}) \oplus \mathbb{Z} \delta$ is the natural Hodge isometry with $\delta$ half the class of the diagonal divisor in $K_{2}(A)$, then

$$
\mathrm{NS}(A)=j \circ \varphi^{\prime-1} \circ \varphi\left(\mathcal{S}_{\iota^{\prime}}\right) .
$$

Then Corollary 7.4 implies that $Y$ is bimeromorphic to $K_{2}(A)$ or to $K_{2}\left(A^{*}\right)$. Let us assume that we have a bimeromorphism $r: Y \rightarrow K_{2}(A)$; if $Y$ is bimeromorphic to $K_{2}\left(A^{*}\right)$, the proof is similar. Then the involution $\iota^{\prime}$ provides an involution $i:=r \circ \iota^{\prime} \circ r^{-1}$, not necessarily regular on $K_{2}(A)$.

On the other hand, by $(7.1)$, we have $\mathrm{NS}(A) \simeq(-2)^{\oplus 2}$. We now construct an involution $g$ on $H^{2}(A, \mathbb{Z})$ given by - id on $(-2)^{\oplus 2}$ and id on $\left((-2)^{\oplus 2}\right)^{\perp}$ and extended to an involution on $H^{2}(A, \mathbb{Z})$ by [Nik80, Corollary 1.5.2]. Then by [Shi78, Theorem 1], the involution $g$ provides a symplectic automorphism on $A$ with $H^{2}(A, \mathbb{Z})^{g}=\left((-2)^{\oplus 2}\right)^{\perp}=U \oplus(2)^{\oplus 2}$. It follows from the classification of [MTW15, Section 4] that $A=\mathbb{C} / \Lambda$ with $\Lambda=\langle(1,0),(0,1),(x,-y),(y, x)\rangle,(x, y) \in \mathbb{C}^{2} \backslash \mathbb{R}^{2}$ and $g=\left(\begin{array}{cc}0 & -1 \\ 1 & 0\end{array}\right)$. We are going to show that $g \circ i$ acts trivially on $H^{2}\left(K_{2}(A), \mathbb{Z}\right)$. The automorphisms $g$ and $i$ are both symplectic, so act trivially on $T_{A}:=\operatorname{NS}\left(K_{2}(A)\right)^{\perp}$, the transcendental lattice of $K_{2}(A)$. Hence, we only have to prove that $g \circ i$ acts trivially on $\mathrm{NS}\left(K_{2}(A)\right)$. We have $\mathrm{NS}\left(K_{2}(A)\right)=j^{-1}(\mathrm{NS}(A)) \oplus \mathbb{Z} \delta \simeq(-2)^{\oplus 2} \oplus(-6)$. We know that $g$ acts on $\mathrm{NS}\left(K_{2}(A)\right)$ by fixing $\delta$ and on $j^{-1}(\mathrm{NS}(A))$ by - id. Moreover, we know that $\mathcal{S}_{i}:=\left(H^{2}\left(K_{2}(A), \mathbb{Z}\right)^{i}\right)^{\perp} \subset \mathrm{NS}\left(K_{2}(A)\right)$ and $\mathcal{S}_{i} \simeq(-2)^{\oplus 2}$. Let $(\alpha, \beta)$ be a basis of $\mathcal{S}_{i}$; we can write $i(\delta)=\lambda \delta+\mu_{1} \alpha+\mu_{2} \beta$ with $\lambda, \mu_{1}$, $\mu_{2}$ integers. Then $i(\delta)^{2}=-6=-6 \lambda^{2}-2 \mu_{1}^{2}-2 \mu_{2}^{2}$, so, necessarily, $\lambda=1$ and $\mu_{1}=\mu_{2}=0$. This implies that $i(\delta)=\delta$ and $\mathcal{S}_{i}=j^{-1}(\mathrm{NS}(A))$. This proves that $g \circ i$ acts trivially on $H^{2}\left(K_{2}(A), \mathbb{Z}\right)$.

Hence, by [Fuj81, Corollary 3.3 and Lemma 3.4], the composition $g \circ i$ extends to an automorphism of $K_{2}(A)$. In particular, $i=g^{-1} \circ g \circ i$ extends to a symplectic involution on $K_{2}(A)$ and $g \circ i \in \operatorname{Ker} \nu$. This allows us to compare the action of $i$ and $g$ on $H^{3}\left(K_{2}(A), \mathbb{Z}\right)$.

By Corollary 6.3 , the translation $t_{\tau}$ acts trivially on $H^{3}\left(K_{2}(A), \mathbb{Z}\right)$. Hence, by Corollary 7.1 , 


\section{S. Kapfer and G. Menet}

we necessarily have

$$
g_{\mid H^{3}\left(K_{2}(A), \mathbb{Z}\right)}^{*}=i_{\mid H^{3}\left(K_{2}(A), \mathbb{Z}\right)}^{*} \circ\left(-\mathrm{id}_{A}\right)_{\mid H^{3}\left(K_{2}(A), \mathbb{Z}\right)}^{*} \text { or } g_{\mid H^{3}\left(K_{2}(A), \mathbb{Z}\right)}^{*}=i_{\mid H^{3}\left(K_{2}(A), \mathbb{Z}\right)}^{*} .
$$

But by Corollary 6.3, the morphism $g_{\mid H^{3}\left(K_{2}(A), \mathbb{Z}\right)}^{*}$ has order 4 , and $i_{\mid H^{3}\left(K_{2}(A), \mathbb{Z}\right)}^{*} \circ\left(-\mathrm{id}_{A}\right)_{\mid H^{3}\left(K_{2}(A), \mathbb{Z}\right)}^{*}$ and $i_{\mid H^{3}\left(K_{2}(A), \mathbb{Z}\right)}^{*}$ have order 2 , which gives a contradiction.

Proof of part (ii). Let $X$ be a irreducible symplectic fourfold of Kummer type and $\iota$ a symplectic involution on $X$. By part (i) of the theorem, we have $\iota \in \operatorname{Ker} \nu$. Then, by [HT13, Theorem 2.1], the pair $(X, \iota)$ deforms to a pair $\left(K_{2}(A), \iota^{\prime}\right)$ with $A$ an abelian surface and $\iota^{\prime} \in \operatorname{Ker} \nu$ a symplectic involution on $K_{2}(A)$. We conclude with Corollary 7.1.

Proof of part (iii). Let $A$ be an abelian surface. By [Tar15, Section 1.2.1], the fixed locus of $t_{\tau} \circ\left(-\mathrm{id}_{A}\right)^{[[3]]}$ on $K_{2}(A)$ consists of a $\mathrm{K} 3$ surface and 36 isolated points. Now, let $X$ be an irreducible symplectic fourfold of Kummer type and $\iota$ a symplectic involution on $X$. By part (ii) of the theorem, the fixed locus Fix $\iota$ deforms to the disjoint union of a K3 surface and 36 isolated points. Moreover, $\iota$ is a symplectic involution, so the holomorphic 2 -form of $X$ restricts to a non-degenerate holomorphic 2-form on Fix $\iota$. So Fix $\iota$ can only contain K3 surfaces, complex tori and isolated points. It follows, necessarily for topological reasons, that Fix $\iota$ consists of a K3 surface and 36 isolated points.

Remark 7.6. With the same ideas as in proof of Theorem 7.5(i), when $n+1$ is a prime power, we can show that a numerically standard symplectic automorphism on an irreducible symplectic $2 n$-fold of Kummer type is standard (see [Mon13] for the definition of standard and numerically standard).

Remark 7.7. (1) We also observe that the K3 surface fixed by $\left(t_{\tau} \circ\left(-\mathrm{id}_{A}\right)\right)$ is given by the submanifold

$$
Z_{-\tau}=\overline{\left\{\left(a_{1}, a_{2}, a_{3}\right) \mid a_{1}=-\tau, a_{2}=-a_{3}+\tau, a_{2} \neq-\tau\right\}}
$$

defined in [HT13, Section 4].

(2) By considering the involution $-\mathrm{id}_{A}$, we see that the set

$$
\mathcal{P}:=\left\{\xi \in K_{2}(A) \mid \operatorname{Supp} \xi=\left\{a_{1}, a_{2}, a_{3}\right\}, a_{i} \in A[2] \backslash\{0\}, 1 \leqslant i \leqslant 3\right\}
$$

provides 35 fixed points and the vertex of $W_{0}:=\left\{\xi \in K_{2}(A) \mid \operatorname{Supp} \xi=\{0\}\right\}$ supplies the 36th point. We denote the points of $\mathcal{P}$ by $p_{1}, \ldots, p_{35}$ and the vertex of $W_{0}$ by $p_{36}$.

\subsection{Action on the cohomology}

By Theorem 7.5, we can assume that $X=K_{2}(A)$ and $\iota=-\mathrm{id}_{A}$. To consider $t_{\tau} \circ\left(-\mathrm{id}_{A}\right)$ instead of $-\mathrm{id}_{A}$ only has the effect of exchanging the role of $\left[Z_{0}\right]$ and $\left[Z_{-\tau}\right]$. Hence, we do not lose any generality assuming that $\iota=-\mathrm{id}_{A}$. We now calculate the invariants $l_{i}^{j}\left(K_{2}(A)\right)$ defined in Definition-Proposition 2.3. This will be used in Section 8.

By Theorem 7.5(i), the involution $\iota$ acts trivially on $H^{2}\left(K_{2}(A), \mathbb{Z}\right)$. It follows that

$$
l_{2}^{2}\left(K_{2}(A)\right)=l_{1,-}^{2}\left(K_{2}(A)\right)=0 \quad \text { and } \quad l_{1,+}^{2}\left(K_{2}(A)\right)=7 .
$$

By Corollary 6.3, the involution $\iota$ acts as $-\mathrm{id}$ on $H^{3}\left(K_{2}(A), \mathbb{Z}\right)$. It follows that

$$
l_{2}^{3}\left(K_{2}(A)\right)=l_{1,+}^{3}\left(K_{2}(A)\right)=0 \quad \text { and } \quad l_{1,-}^{3}\left(K_{2}(A)\right)=8 .
$$

By Definition 6.21, we have

$$
H^{4}\left(K_{2}(A), \mathbb{Q}\right)=\operatorname{Sym}^{2} H^{2}\left(K_{2}(A), \mathbb{Q}\right) \oplus^{\perp} \Pi^{\prime} \otimes \mathbb{Q},
$$


where $\Pi^{\prime}=\left\langle Z_{\tau}-Z_{0}, \tau \in A[3] \backslash\{0\}\right\rangle$. The involution $\iota^{*}$ fixes $\operatorname{Sym}^{2} H^{2}\left(K_{2}(A), \mathbb{Z}\right)$ and $\iota^{*}\left(Z_{\tau}-\right.$ $\left.Z_{0}\right)=Z_{-\tau}-Z_{0}$. This leads to the following proposition.

Proposition 7.8. We have $l_{1,-}^{4}\left(K_{2}(A)\right)=0, l_{1,+}^{4}\left(K_{2}(A)\right)=28$ and $l_{2}^{4}\left(K_{2}(A)\right)=40$.

Proof. Let $\mathcal{S}$ be the overlattice of $\operatorname{Sym}^{2} H^{2}\left(K_{2}(A), \mathbb{Z}\right)$, where we add all the classes divisible by 2 in $H^{4}\left(K_{2}(A), \mathbb{Z}\right)$. From Section 6.6, we know that the discriminant of $\mathcal{S}$ is not divisible by 2. Hence, we have $H^{4}\left(K_{2}(A), \mathbb{F}_{2}\right)=\mathcal{S} \otimes \mathbb{F}_{2} \oplus \Pi^{\prime} \otimes \mathbb{F}_{2}$. Moreover, we have $\iota^{*}\left(Z_{\tau}-Z_{0}\right)=Z_{-\tau}-Z_{0}$ for all $\tau \in A[3] \backslash\{0\}$. Hence, $\operatorname{Vect}_{\mathbb{F}_{2}}\left(Z_{\tau}-Z_{0}, Z_{-\tau}-Z_{0}\right)$ is isomorphic to $N_{2}$ as an $\mathbb{F}_{2}[G]$-module (see the notation in Definition-Proposition 2.3). Moreover, $H^{2}\left(K_{2}(A), \mathbb{Z}\right)$ is invariant under the action of $\iota$; hence, $\operatorname{Sym}^{2} H^{2}\left(K_{2}(A), \mathbb{Z}\right)$ and $\mathcal{S}$ are also invariant under the action of $\iota$. It follows that $\mathcal{S} \otimes \mathbb{F}_{2}=\mathcal{N}_{1}$ and $\Pi^{\prime} \otimes \mathbb{F}_{2}=\mathcal{N}_{2}$. Since rk $\mathcal{S}=28$, we have $l_{1,+}^{4}+l_{1,-}^{4}=28$. However, $\mathcal{S}$ is invariant under the action of $\iota$; it follows that $l_{1,-}^{4}=0$ and $l_{1,+}^{4}=28$. On the other hand, $\operatorname{rk} \Pi^{\prime}=80$; it follows that $l_{2}^{4}=40$.

\section{Proof of Theorem 1.1}

Since all generalized Kummer fourfolds are deformation equivalent, by Theorem 7.5, all pairs $(X, \iota)$, where $X$ is a fourfold of Kummer type and $\iota$ a symplectic involution, are deformation equivalent. Moreover, the Beauville-Bogomolov form is a topological invariant; hence, without loss of generality, it is enough to prove Theorem 1.1 for a particular pair $(X, \iota)$. We can assume that $X$ is a generalized Kummer fourfold and $\iota=-\mathrm{id}_{A}$. As it will be useful in proving Lemma 8.17, we can, moreover, assume that $A=E_{\xi} \times E_{\xi}$, where

$$
E_{\xi}:=\frac{\mathbb{C}}{\Lambda_{0}}
$$

with $\xi:=e^{2 i \pi / 6}$ and $\Lambda_{0}:=\langle 1, \xi\rangle$. This abelian surface has the interest of carrying enough automorphisms.

Definition 8.1. Define the group $G_{\xi}$ of automorphisms of $E_{\xi} \times E_{\xi}$ by the following generators in $\operatorname{GL}\left(2, \operatorname{End}\left(\Lambda_{0}\right)\right)$ :

$$
g_{1}=\left(\begin{array}{cc}
\xi & 0 \\
0 & 1
\end{array}\right), \quad g_{2}=\left(\begin{array}{ll}
0 & 1 \\
1 & 0
\end{array}\right), \quad g_{3}=\left(\begin{array}{ll}
1 & 1 \\
0 & 1
\end{array}\right)
$$

For $A=E_{\xi} \times E_{\xi}$, let $V=A[2]$ be the (4-dimensional) $\mathbb{F}_{2}$-vector space of 2-torsion points on $A$, and let $\mathfrak{T}$ be the set of planes in $V$. Note that by Remark 6.9, a plane in $V$ can be identified with an unordered triple $\{x, y, z\}$ with $x, y, z \in V$ non-zero and $x+y+z=0$. The action of $G_{\xi}$ on $A$ induces actions of $G_{\xi}$ on $A[2]$ and $\mathfrak{T}$. The following lemma will be used to prove Theorem 8.12.

Lemma 8.2. There are two orbits of $G_{\xi}$ on $\mathfrak{T}$, of cardinalities 5 and 30.

Proof. Note that the generators $g_{2}$ and $g_{3}$ exist because $A$ is of the form $E \times E$, while $g_{1}$ exists only in the special case $E=E_{\xi}$. Indeed, multiplication with $\xi$ induces a cyclic permutation on $E_{\xi}[2]$. The orbits can be determined explicitly by a suitable computer program. For verification, we give one of the orbits explicitly. Let $x_{1}, x_{2}, x_{3}$ be the non-zero points in $E_{\xi}[2]$. The orbit of cardinality 5 is then given by

$$
\begin{gathered}
\left\{\left(0, x_{1}\right),\left(0, x_{2}\right),\left(0, x_{3}\right)\right\}, \quad\left\{\left(x_{1}, 0\right),\left(x_{2}, 0\right),\left(x_{3}, 0\right)\right\}, \quad\left\{\left(x_{1}, x_{1}\right),\left(x_{2}, x_{2}\right),\left(x_{3}, x_{3}\right)\right\}, \\
\left\{\left(x_{1}, x_{2}\right),\left(x_{2}, x_{3}\right),\left(x_{3}, x_{1}\right)\right\}, \quad\left\{\left(x_{1}, x_{3}\right),\left(x_{2}, x_{1}\right),\left(x_{3}, x_{2}\right)\right\} .
\end{gathered}
$$




\section{S. Kapfer And G. Menet}

\subsection{Overview on the proof of Theorem 1.1 and notation}

The proof is divided into the following steps:

(1) First (7.2), (7.3), Proposition 7.8 and Theorem 8.7 will provide the $H^{4}$-normality of $\left(K_{2}(A), \iota\right)$ in Section 8.2. The notion of $H^{k}$-normality is recalled at the beginning of the section.

(2) The knowledge of the elements divisible by 2 in $\operatorname{Sym}^{2} H^{2}\left(K_{2}(A), \mathbb{Z}\right)$ from Section 6.6 and the $H^{4}$-normality allow us to prove the $H^{2}$-normality of $\left(K_{2}(A), \iota\right)$ in Section 8.3.

(3) An adaptation of the $H^{2}$-normality (Lemma 8.11) and several lemmas in Section 8.4 will provide an integral basis of $H^{2}\left(K^{\prime}, \mathbb{Z}\right)$ (Theorem 8.12).

(4) Knowing an integral basis of $H^{2}\left(K^{\prime}, \mathbb{Z}\right)$, we complete the calculation of the BeauvilleBogomolov form in Section 8.5 using intersection theory and the Fujiki formula.

We now provide some notation that we will be used during the proof. Let $K_{2}(A)$ be a generalized Kummer fourfold endowed with the symplectic involution $\iota$ induced by $-\mathrm{id}_{A}$. We denote the quotient map $K_{2}(A) \rightarrow K_{2}(A) / \iota$ by $\pi$. From Theorem 7.5 , we know that the singular locus of the quotient $K_{2}(A) / \iota$ is the K3 surface that is the image of $Z_{0}$ and 36 isolated points by $\pi$. We set $\overline{Z_{0}}:=\pi\left(Z_{0}\right)$. We consider $r^{\prime}: K^{\prime} \rightarrow K_{2}(A) / \iota$, the blow-up of $K_{2}(A) / \iota$ in $\overline{Z_{0}}$; we denote the exceptional divisor by ${\overline{Z_{0}}}^{\prime}$. We also consider $s_{1}: N_{1} \rightarrow K_{2}(A)$, the blow-up of $K_{2}(A)$ in $Z_{0}$; we denote the exceptional divisor in $N_{1}$ by $Z_{0}^{\prime}$. Let $\iota_{1}$ be the involution on $N_{1}$ induced by $\iota$. We have $K^{\prime} \simeq N_{1} / \iota_{1}$; we denote the quotient map by $\pi_{1}: N_{1} \rightarrow K^{\prime}$.

Consider the blow-up $s_{2}: N_{2} \rightarrow N_{1}$ of $N_{1}$ in the 36 points $p_{1}, \ldots, p_{36}$ fixed by $\iota_{1}$ and the blow-up $\widetilde{r}: \widetilde{K} \rightarrow K^{\prime}$ of $K^{\prime}$ in its 36 singular points. We denote the exceptional divisors by $E_{1}, \ldots, E_{36}$ and $D_{1}, \ldots, D_{36}$, respectively. We also write $\widetilde{Z_{0}}=\widetilde{r}^{*}\left({\overline{Z_{0}}}^{\prime}\right)$ and $\widetilde{Z_{0}}=s_{2}^{*}\left(Z_{0}^{\prime}\right)$. We denote the involution induced by $\iota$ on $N_{2}$ by $\iota_{2}$ and the quotient map by $\pi_{2}: N_{2} \rightarrow N_{2} / \iota_{2}$. We have $N_{2} / \iota_{2} \simeq \widetilde{K}$. To finish, we set $V=K_{2}(A) \backslash$ Fix $\iota$ and $U=V / \iota$. We collect this notation in a commutative diagram:

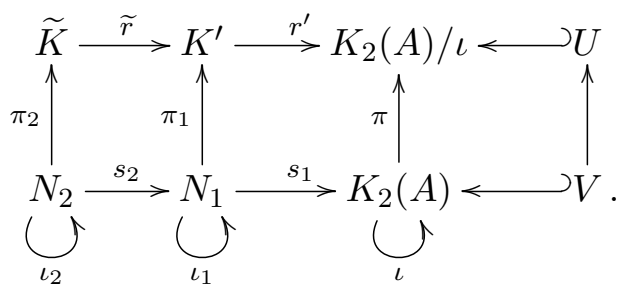

We set $s=s_{2} \circ s_{1}$ and $r=\widetilde{r} \circ r^{\prime}$. We denote the half of the class of the diagonal in $H^{2}\left(K_{2}(A), \mathbb{Z}\right)$ by $e$, as in Notation 5.13 .

Remark 8.3. We can commute the push-forward maps and the blow-up maps, as proved in [Men14, Lemma 3.3.21]. Let $x \in H^{2}\left(N_{1}, \mathbb{Z}\right)$ and $y \in H^{2}\left(K_{2}(A), \mathbb{Z}\right)$; we have

$$
\pi_{2 *}\left(s_{2}^{*}(x)\right)=\widetilde{r}^{*}\left(\pi_{1 *}(x)\right), \quad \pi_{1 *}\left(s_{1}^{*}(y)\right)=r^{\prime *}\left(\pi_{*}(y)\right) .
$$

Moreover, we will also use the notation provided in Notation 5.13 and Section 6.4.

\section{2 $H^{4}$-normality of the pair $\left(K_{2}(A), \iota\right)$}

We will use the notion of $H^{k}$-normality from [Men14, Definition 3.3.4] that we recall here.

DeFinition 8.4. Let $X$ be a compact complex manifold and $\iota$ an involution. Let $0 \leqslant k \leqslant 2 n$, and assume that $H^{k}(X, \mathbb{Z})$ is torsion free. Then if the map $\pi_{*}: H^{k}(X, \mathbb{Z}) \rightarrow H^{k}(X / G, \mathbb{Z}) /$ tors is surjective, we say that $(X, \iota)$ is $H^{k}$-normal. 


\section{INTEGRAL COHOMOLOGY OF THE GENERALIZED KUMMER FOURFOLD}

Remark 8.5. The $H^{k}$-normal property is equivalent to the following property: for $x \in H^{k}(X, \mathbb{Z})^{\iota}$, the image $\pi_{*}(x)$ is divisible by 2 if and only if there exists a $y \in H^{k}(X, \mathbb{Z})$ such that $x=y+\iota^{*}(y)$.

Definition 8.6. Let $X$ be a compact complex manifold of dimension $n$ and $G$ an automorphism group of prime order $p$.

(i) We will say that Fix $G$ is negligible if the following conditions are verified:

- The group $H^{*}(\operatorname{Fix} G, \mathbb{Z})$ is torsion free.

- $\operatorname{Codim}$ Fix $G \geqslant n / 2+1$.

(ii) We will say that $\operatorname{Fix} G$ is almost negligible if the following conditions are verified:

- The group $H^{*}(\operatorname{Fix} G, \mathbb{Z})$ is torsion free.

- The dimension $n$ is even and at least 4 .

- We have Codim Fix $G=n / 2$, and the purely $n / 2$-dimensional part of Fix $G$ is connected and simply connected. We denote the $n / 2$-dimensional component by $Z$.

- The cocycle $[Z]$ associated with $Z$ is primitive in $H^{n}(X, \mathbb{Z})$.

We will use the following theorem ([Men14, Corollary 3.5.18]) to prove the $H^{4}$-normality of $\left(K_{2}(A), \iota\right)$.

THEOREM 8.7. Let $G$ be a group of order 2 acting by automorphisms on a Kähler manifold $X$ of dimension $2 n$. We assume that

(i) $H^{*}(X, \mathbb{Z})$ is torsion free,

(ii) Fix $G$ is negligible or almost negligible,

(iii) $l_{1,-}^{2 k}(X)=0$ for all $1 \leqslant k \leqslant n$,

(iv) $l_{1,+}^{2 k+1}(X)=0$ for all $0 \leqslant k \leqslant n-1$ when $n>1$,

(v) $l_{1,+}^{2 n}(X)+2\left[\sum_{i=0}^{n-1} l_{1,-}^{2 i+1}(X)+\sum_{i=0}^{n-1} l_{1,+}^{2 i}(X)\right]=\sum_{k=0}^{\operatorname{dim} \operatorname{Fix} G} h^{2 k}($ Fix $G, \mathbb{Z})$.

Then $(X, G)$ is $H^{2 n}$-normal.

Proposition 8.8. The pair $\left(K_{2}(A), \iota\right)$ is $H^{4}$-normal.

Proof. We apply Theorem 8.7. Let us check the assumptions.

(i) By Theorem 5.2, the group $H^{*}\left(K_{2}(A), \mathbb{Z}\right)$ is torsion free.

(ii) From Remark 7.7(1), we know that the connected component of dimension 2 of Fix $\iota$ is given by $Z_{0}$, which is a K3 surface, hence is simply connected. Moreover, by [HT13, Proposition 4.3], we have $Z_{0} \cdot Z_{\tau}=1$ for all $\tau \in A[3] \backslash\{0\}$. Hence, the class of $Z_{0}$ in $H^{4}\left(K_{2}(A), \mathbb{Z}\right)$ is primitive. It follows that Fix $\iota$ is almost negligible (Definition 8.6).

(iii) By (7.2) and Proposition 7.8, we have $l_{1,-}^{2}\left(K_{2}(A)\right)=l_{1,-}^{4}\left(K_{2}(A)\right)=0$.

(iv) By $(7.3)$, we have $l_{1,+}^{3}\left(K_{2}(A)\right)=0$. Moreover, $H^{1}\left(K_{2}(A)\right)=0$, so $l_{1,+}^{1}\left(K_{2}(A)\right)=0$.

(v) We have to check the following equality:

$$
\begin{aligned}
l_{1,+}^{4}\left(K_{2}(A)\right) & +2\left[l_{1,-}^{1}(X)+l_{1,-}^{3}(X)+l_{1,+}^{0}(X)+l_{1,+}^{2}(X)\right] \\
& =36 h^{0}(p t)+h^{0}\left(Z_{0}\right)+h^{2}\left(Z_{0}\right)+h^{4}\left(Z_{0}\right) .
\end{aligned}
$$

By (7.2), (7.3) and Proposition 7.8,

$$
l_{1,+}^{4}\left(K_{2}(A)\right)+2\left[l_{1,-}^{1}(X)+l_{1,-}^{3}(X)+l_{1,+}^{0}(X)+l_{1,+}^{2}(X)\right]=28+2(8+1+7)=60 .
$$




\section{S. Kapfer and G. Menet}

Moreover, since $Z_{0}$ is a $\mathrm{K} 3$ surface, we have

$$
36 h^{0}(p t)+h^{0}\left(Z_{0}\right)+h^{2}\left(Z_{0}\right)+h^{4}\left(Z_{0}\right)=36+1+22+1=60 .
$$

It follows from Theorem 8.7 that $\left(K_{2}(A), \iota\right)$ is $H^{4}$-normal.

Remark 8.9. As explained in [Men14, Proposition 3.5.20], the proof of Theorem 8.7 provide first that $\pi_{2 *}\left(s^{*}\left(H^{4}\left(K_{2}(A), \mathbb{Z}\right)\right)\right)$ is primitive in $H^{4}(\widetilde{K}, \mathbb{Z})$ and then the $H^{4}$-normality. So, the lattice $\pi_{2 *}\left(s^{*}\left(H^{4}\left(K_{2}(A), \mathbb{Z}\right)\right)\right)$ is primitive in $H^{4}(\widetilde{K}, \mathbb{Z})$.

\section{3 $H^{2}$-normality of the pair $\left(K_{2}(A), \iota\right)$}

Proposition 8.10. The pair $\left(K_{2}(A), \iota\right)$ is $H^{2}$-normal.

Proof. We want to prove that the push-forward $\pi_{*}: H^{2}\left(K_{2}(A), \mathbb{Z}\right) \rightarrow H^{2}\left(K_{2}(A) / \iota, \mathbb{Z}\right) /$ tors is surjective. By Remark 8.5, it is equivalent to prove that for all $x \in H^{2}\left(K_{2}(A), \mathbb{Z}\right)^{\iota}$, the image $\pi_{*}(x)$ is divisible by 2 if and only if there exists a $y \in H^{2}\left(K_{2}(A), \mathbb{Z}\right)$ such that $x=y+\iota^{*}(y)$.

Let $x \in H^{2}\left(K_{2}(A), \mathbb{Z}\right)^{\iota}=H^{2}\left(K_{2}(A), \mathbb{Z}\right)$ be such that $\pi_{*}(x)$ is divisible by 2 ; we will show that there exists a $y \in H^{2}\left(K_{2}(A), \mathbb{Z}\right)$ such that $x=y+\iota^{*}(y)$. By Proposition 2.7, the image $\pi_{*}\left(x^{2}\right)$ is divisible by 2 . However, $x^{2} \in H^{4}\left(K_{2}(A), \mathbb{Z}\right)^{\iota}$; since $\left(K_{2}(A), \iota\right)$ is $H^{4}$-normal by Proposition 8.8, this means that there exists a $z \in H^{4}\left(K_{2}(A), \mathbb{Z}\right)$ such that $x^{2}=z+\iota^{*}(z)$.

As before, let $\mathcal{S}$ be the overlattice of $\operatorname{Sym}^{2} H^{2}\left(K_{2}(A), \mathbb{Z}\right)$, where we add all the classes divisible by 2 in $H^{4}\left(K_{2}(A), \mathbb{Z}\right)$. By Definition 6.21 and $(6.13)$, there exist $z_{s} \in \mathcal{S}, z_{p} \in \Pi^{\prime}$ and $\alpha \in \mathbb{N}$ such that $3^{\alpha} \cdot z=z_{s}+z_{p}$. Hence, we have $3^{\alpha} \cdot x^{2}=2 z_{s}+z_{p}+\iota^{*}\left(z_{p}\right)$. Since $x^{2} \in$ Sym, we have $z_{p}+\iota^{*}\left(z_{p}\right)=0$. It follows that

$$
3^{\alpha} \cdot x^{2}=2 z_{s} .
$$

Let $\left(u_{1}, u_{2}, v_{1}, v_{2}, w_{1}, w_{2}, e\right)$ be the integral basis of $H^{2}\left(K_{2}(A), \mathbb{Z}\right)$ introduced in Notation 5.13. We can write

$$
x=\alpha_{1} u_{1}+\alpha_{2} u_{2}+\beta_{1} v_{1}+\beta_{2} v_{2}+\gamma_{1} w_{1}+\gamma_{2} w_{2}+d e .
$$

Then

$$
3^{\alpha} \cdot x^{2}=\alpha_{1}^{2} u_{1}^{2}+\alpha_{2}^{2} u_{2}^{2}+\beta_{1}^{2} v_{1}^{2}+\beta_{2}^{2} v_{2}^{2}+\gamma_{1}^{2} w_{1}^{2}+\gamma_{2}^{2} w_{2}^{2}+d^{2} e^{2} \quad \bmod 2 H^{4}\left(K_{2}(A), \mathbb{Z}\right) .
$$

It follows by (8.2) that $\alpha_{1}^{2} u_{1}^{2}+\alpha_{2}^{2} u_{2}^{2}+\beta_{1}^{2} v_{1}^{2}+\beta_{2}^{2} v_{2}^{2}+\gamma_{1}^{2} w_{1}^{2}+\gamma_{2}^{2} w_{2}^{2}+d^{2} e^{2}$ is divisible by 2 . However, by Theorem $6.31(\mathrm{i})$, we have

$$
\begin{aligned}
\mathcal{S}=\left\langle\operatorname{Sym}^{2} H^{2}\left(K_{2}(A), \mathbb{Z}\right) ; \frac{u_{1} \cdot u_{2}+v_{1} \cdot v_{2}+w_{1} \cdot w_{2}}{2}\right. & \\
\frac{u_{i}^{2}-\frac{1}{3} u_{i} \cdot e}{2} & \left.; \frac{v_{i}^{2}-\frac{1}{3} v_{i} \cdot e}{2} ; \frac{w_{i}^{2}-\frac{1}{3} w_{i} \cdot e}{2}, i \in\{1,2\}\right\rangle .
\end{aligned}
$$

The sum $\frac{1}{2}\left(\alpha_{1}^{2} u_{1}^{2}+\alpha_{2}^{2} u_{2}^{2}+\beta_{1}^{2} v_{1}^{2}+\beta_{2}^{2} v_{2}^{2}+\gamma_{1}^{2} w_{1}^{2}+\gamma_{2}^{2} w_{2}^{2}+d^{2} e^{2}\right)$ is in $\mathcal{S}$ and so can be expressed as a linear combination of the generators of $\mathcal{S}$. Then it follows from (8.3) that all the coefficients of $\alpha_{1}^{2} u_{1}^{2}+\alpha_{2}^{2} u_{2}^{2}+\beta_{1}^{2} v_{1}^{2}+\beta_{2}^{2} v_{2}^{2}+\gamma_{1}^{2} w_{1}^{2}+\gamma_{2}^{2} w_{2}^{2}+d^{2} e^{2}$ are divisible by 2 . This means that $x$ is divisible by 2 . This is what we wanted to prove.

With exactly the same proof working in $H^{4}(\widetilde{K}, \mathbb{Z})$, using Remark 8.9, we provide the following lemma.

Lemma 8.11. The lattice $\pi_{2 *}\left(s^{*}\left(H^{2}\left(K_{2}(A), \mathbb{Z}\right)\right)\right)$ is primitive in $H^{2}(\widetilde{K}, \mathbb{Z})$. 


\section{INTEGRAL COHOMOLOGY OF THE GENERALIZED KUMMER FOURFOLD}

\subsection{Calculation of $H^{2}\left(K^{\prime}, \mathbb{Z}\right)$}

This section is devoted to proving the following theorem.

THEOREM 8.12. Let $K^{\prime}, \pi_{1}, s_{1}$ and ${\overline{Z_{0}}}^{\prime}$ be, respectively, the variety, the maps and the class defined in Section 8.1. We have

$$
H^{2}\left(K^{\prime}, \mathbb{Z}\right)=\pi_{1 *}\left(s_{1}^{*}\left(H^{2}\left(K_{2}(A), \mathbb{Z}\right)\right)\right) \oplus \mathbb{Z}\left(\frac{\pi_{1 *}\left(s_{1}^{*}(e)\right)+{\overline{Z_{0}}}^{\prime}}{2}\right) \oplus \mathbb{Z}\left(\frac{\pi_{1 *}\left(s_{1}^{*}(e)\right)-{\overline{Z_{0}}}^{\prime}}{2}\right) .
$$

First we need to calculate some intersections.

LEMma 8.13. (i) We have $E_{l} \cdot E_{k}=0$ if $l \neq k, E_{l}^{4}=-1$ and $E_{l} \cdot z=0$ for all $(l, k) \in\{1, \ldots, 28\}^{2}$ and for all $z \in s^{*}\left(H^{2}\left(K_{2}(A), \mathbb{Z}\right)\right)$.

(ii) We have $e^{4}=324$.

We already have some primitivity properties:

(iii) The class $\pi_{1 *}\left(s_{1}^{*}\left(H^{2}\left(K_{2}(A), \mathbb{Z}\right)\right)\right)$ is primitive in $H^{2}\left(K^{\prime}, \mathbb{Z}\right)$,

(iv) The group $\widetilde{\mathcal{D}}=\left\langle\widetilde{\overline{Z_{0}}}, D_{1}, \ldots, D_{36}, \frac{1}{2}\left(\widetilde{\overline{Z_{0}}}+D_{1}+\cdots+D_{36}\right)\right\rangle$ is primitive in $H^{2}(\widetilde{K}, \mathbb{Z})$.

(v) The class ${\overline{Z_{0}}}^{\prime}$ is primitive in $H^{2}\left(K^{\prime}, \mathbb{Z}\right)$.

Proof. (i) This is the same statement as [Men14, Proposition 4.6.16 1)], proven using the adjunction formula.

(ii) This follows directly from the Fujiki formula (5.5).

(iii) By Lemma 8.11, the class $\pi_{2 *}\left(s^{*}\left(H^{2}\left(K_{2}(A), \mathbb{Z}\right)\right)\right)$ is primitive in $H^{2}(\widetilde{K}, \mathbb{Z})$. Then by Remark 8.3, the class $r^{*}\left(\pi_{*}\left(H^{2}\left(K_{2}(A), \mathbb{Z}\right)\right)\right)$ is primitive in $H^{2}\left(K^{\prime}, \mathbb{Z}\right)$. Using Remark 8.3 again, we get the result.

The proof of parts (iv) and (v) is the same as that of [Men14, Lemma 4.6.14] and will be omitted.

With Lemma 8.13(iii) and (v), it only remains to prove that $\pi_{1 *}\left(s_{1}^{*}(e)\right)+{\overline{Z_{0}}}^{\prime}$ is divisible by 2 , which will be done in Lemma 8.18. To prove this lemma, we first prove that $\pi_{2 *}\left(s^{*}(e)\right)+\widetilde{\bar{Z}_{0}}$ is divisible by 2 . Knowing that $\widetilde{Z_{0}}+D_{1}+\cdots+D_{36}$ is divisible by 2 , we only have to show that $\pi_{2 *}\left(s^{*}(e)\right)+D_{1}+\cdots+D_{36}$ is divisible by 2 , which is done by Lemmas 8.16 and 8.17.

First we need to know the group $H^{3}(\widetilde{K}, \mathbb{Z})$.

LEMma 8.14. We have $H^{3}(\widetilde{K}, \mathbb{Z})=0$.

Proof. We have the following exact sequence:

$$
H^{3}\left(K_{2}(A), V, \mathbb{Z}\right) \longrightarrow H^{3}\left(K_{2}(A), \mathbb{Z}\right) \stackrel{f}{\longrightarrow} H^{3}(V, \mathbb{Z}) \longrightarrow H^{4}\left(K_{2}(A), V, \mathbb{Z}\right) \stackrel{\varrho}{\longrightarrow} H^{4}\left(K_{2}(A), \mathbb{Z}\right) .
$$

By the Thom isomorphism, $H^{3}\left(K_{2}(A), V, \mathbb{Z}\right)=0$ and $H^{4}\left(K_{2}(A), V, \mathbb{Z}\right)=H^{0}\left(Z_{0}, \mathbb{Z}\right)$. Moreover, $\varrho$ is injective, so $H^{3}(V, \mathbb{Z})=H^{3}\left(K_{2}(A), \mathbb{Z}\right)$.

Hence, by (7.2), (7.3), [Men14, Proposition 3.2.8] and the identity $H^{3}\left(K_{2}(A), \mathbb{Z}\right)^{\iota}=0$, we find that $H^{3}(U, \mathbb{Z})=0$. The result then follows from the exact sequence

$$
H^{3}(\widetilde{K}, U, \mathbb{Z}) \longrightarrow H^{3}(\widetilde{K}, \mathbb{Z}) \longrightarrow H^{3}(U, \mathbb{Z})
$$

and from the fact that $H^{3}(\widetilde{K}, U, \mathbb{Z})=0$ by the Thom isomorphism. 


\section{S. Kapfer And G. Menet}

To prove the next lemma, we will need a proposition from [BNS13, Section 7] about Smith theory. Let $X$ be a topological space, and let $G=\langle\iota\rangle$ be an involution acting on $X$. Let $\sigma:=1+\iota \in$ $\mathbb{F}_{2}[G]$. We consider the chain complex $C_{*}(X)$ of $X$ with coefficients in $\mathbb{F}_{2}$ and its subcomplex $\sigma C_{*}(X)$. We denote the fixed locus of the action of $G$ on $X$ by $X^{G}$.

Proposition 8.15. (i) ([Bre72, Theorem 3.1]). There is an exact sequence of complexes

$$
0 \longrightarrow \sigma C_{*}(X) \oplus C_{*}\left(X^{G}\right) \stackrel{f}{\longrightarrow} C_{*}(X) \stackrel{\sigma}{\longrightarrow} \sigma C_{*}(X) \longrightarrow 0,
$$

where $f$ denotes the sum of the inclusions.

(ii) $([$ Bre72, (3.4), p. 124]). There is an isomorphism of complexes

$$
\sigma C_{*}(X) \simeq C_{*}\left(X / G, X^{G}\right),
$$

where $X^{G}$ is identified with its image in $X / G$.

Lemma 8.16. There exists a $D_{e}$ which is a linear combination of the $D_{i}$ with coefficients 0 or 1 such that $\pi_{2 *}\left(s^{*}(e)\right)+D_{e}$ is divisible by 2 .

Proof. First we have to use Smith theory as in [Men14, Section 4.6.4].

Look at the following exact sequence:

$$
\begin{aligned}
& 0 \longrightarrow H^{2}\left(\widetilde{K}, \widetilde{Z_{0}} \cup\left(\cup_{k=1}^{36} D_{k}\right), \mathbb{F}_{2}\right) \longrightarrow H^{2}\left(\widetilde{K}, \mathbb{F}_{2}\right) \longrightarrow H^{2}\left(\widetilde{\bar{Z}_{0}} \cup\left(\cup_{k=1}^{36} D_{k}\right), \mathbb{F}_{2}\right) \\
& \longrightarrow H^{3}\left(\widetilde{K}, \widetilde{Z_{0}} \cup\left(\cup_{k=1}^{36} D_{k}\right), \mathbb{F}_{2}\right) \longrightarrow 0 .
\end{aligned}
$$

First, we will calculate the dimension of the vector spaces $H^{2}\left(\widetilde{K}, \widetilde{Z_{0}} \cup\left(\cup_{k=1}^{36} D_{k}\right), \mathbb{F}_{2}\right)$ and $H^{3}\left(\widetilde{K}, \widetilde{Z_{0}} \cup\left(\cup_{k=1}^{36} D_{k}\right), \mathbb{F}_{2}\right)$. By Proposition 8.15(ii), we have

$$
H^{*}\left(\widetilde{K}, \widetilde{Z_{0}} \cup\left(\cup_{k=1}^{36} D_{k}\right), \mathbb{F}_{2}\right) \simeq H_{\sigma}^{*}\left(N_{2}\right) .
$$

The previous exact sequence gives us the following equation:

$$
h_{\sigma}^{2}\left(N_{2}\right)-h^{2}\left(\widetilde{K}, \mathbb{F}_{2}\right)+h^{2}\left(\widetilde{Z_{0}} \cup\left(\cup_{k=1}^{36} D_{k}\right), \mathbb{F}_{2}\right)-h_{\sigma}^{3}\left(N_{2}\right)=0 .
$$

As $h^{2}\left(\widetilde{K}, \mathbb{F}_{2}\right)=8+36=44$ and $h^{2}\left(\widetilde{\bar{Z}_{0}} \cup\left(\cup_{k=1}^{36} D_{k}\right), \mathbb{F}_{2}\right)=23+36=59$, we obtain

$$
h_{\sigma}^{2}\left(N_{2}\right)-h_{\sigma}^{3}\left(N_{2}\right)=-15 \text {. }
$$

Moreover, by Proposition 8.15(ii), we have the exact sequence

$$
\begin{aligned}
0 \longrightarrow H_{\sigma}^{1}\left(N_{2}\right) \longrightarrow H_{\sigma}^{2}\left(N_{2}\right) \longrightarrow H^{2}\left(N_{2}, \mathbb{F}_{2}\right) \longrightarrow H_{\sigma}^{2}\left(N_{2}\right) \oplus H^{2}\left(\widetilde{Z_{0}} \cup\left(\cup_{k=1}^{36} E_{k}\right), \mathbb{F}_{2}\right) \\
\longrightarrow H_{\sigma}^{3}\left(N_{2}\right) \longrightarrow \text { coker } \longrightarrow 0 .
\end{aligned}
$$

By [BNS13, Lemma 7.4], we have $h_{\sigma}^{1}\left(N_{2}\right)=h^{0}\left(\widetilde{Z_{0}} \cup\left(\cup_{k=1}^{36} E_{k}\right), \mathbb{F}_{2}\right)-1$. Then we get the equation

$$
\begin{aligned}
h^{0}\left(\widetilde{Z_{0}} \cup\right. & \left.\left(\cup_{k=1}^{36} E_{k}\right), \mathbb{F}_{2}\right)-1-h_{\sigma}^{2}\left(N_{2}\right)+h^{2}\left(N_{2}, \mathbb{F}_{2}\right) \\
& -h_{\sigma}^{2}\left(N_{2}\right)-h^{2}\left(\widetilde{Z_{0}} \cup\left(\cup_{k=1}^{36} E_{k}\right), \mathbb{F}_{2}\right)+h_{\sigma}^{3}\left(N_{2}\right)-\alpha=0,
\end{aligned}
$$

where $\alpha=\operatorname{dim}$ coker. So $21-\alpha-2 h_{\sigma}^{2}\left(N_{2}\right)+h_{\sigma}^{3}\left(N_{2}\right)=0$. From the two equations, we deduce that

$$
h_{\sigma}^{2}\left(N_{2}\right)=36-\alpha, \quad h_{\sigma}^{3}\left(N_{2}\right)=51-\alpha .
$$




\section{INTEGRAL COHOMOLOGY OF THE GENERALIZED KUMMER FOURFOLD}

We return to the exact sequence

$$
0 \longrightarrow H^{2}\left(\widetilde{K}, \widetilde{Z_{0}} \cup\left(\cup_{k=1}^{36} D_{k}\right), \mathbb{F}_{2}\right) \longrightarrow H^{2}\left(\widetilde{K}, \mathbb{F}_{2}\right) \stackrel{\varsigma^{*}}{\longrightarrow} H^{2}\left(\widetilde{Z_{0}} \cup\left(\cup_{k=1}^{36} D_{k}\right), \mathbb{F}_{2}\right),
$$

where $\varsigma: \widetilde{\overline{Z_{0}}} \cup\left(\cup_{k=1}^{36} D_{k}\right) \hookrightarrow \widetilde{K}$ is the inclusion. Since $h^{2}\left(\widetilde{K}, \widetilde{Z_{0}} \cup\left(\cup_{k=1}^{36} D_{k}\right), \mathbb{F}_{2}\right)=h_{\sigma}^{2}\left(N_{2}\right)=36-\alpha$, we have $\operatorname{dim}_{\mathbb{F}_{2}} \varsigma^{*}\left(H^{2}\left(\widetilde{K}, \mathbb{F}_{2}\right)\right)=(8+36)-36+\alpha=8+\alpha$. We can interpret this as follows. Consider the homomorphism

$$
\begin{aligned}
\varsigma_{\mathbb{Z}}^{*}: H^{2}(\widetilde{K}, \mathbb{Z}) \longrightarrow H^{2}\left(\widetilde{\overline{Z_{0}}}, \mathbb{Z}\right) \oplus\left(\oplus_{k=1}^{36} H^{2}\left(D_{k}, \mathbb{Z}\right)\right), \\
u \longmapsto\left(u \cdot \widetilde{\overline{Z_{0}}}, u \cdot D_{1}, \ldots, u \cdot D_{36}\right) .
\end{aligned}
$$

Since this is a map of torsion-free $\mathbb{Z}$-modules (by Lemma 8.14 and the universal coefficient formula), we can tensor by $\mathbb{F}_{2}$,

$$
\varsigma^{*}=\varsigma_{\mathbb{Z}}^{*} \otimes \operatorname{id}_{\mathbb{F}_{2}}: H^{2}(\widetilde{K}, \mathbb{Z}) \otimes \mathbb{F}_{2} \rightarrow H^{2}\left(\widetilde{Z_{0}}, \mathbb{Z}\right) \oplus\left(\oplus_{k=1}^{36} H^{2}\left(D_{k}, \mathbb{Z}\right)\right) \otimes \mathbb{F}_{2},
$$

and we have $8+\alpha$ independent elements such that the intersection with the $D_{k}$, for $k \in$ $\{1, \ldots, 36\}$, and $\widetilde{Z_{0}}$ are not all zero. But $\varsigma^{*}\left(\pi_{2 *}\left(H^{2}\left(N_{2}, \mathbb{Z}\right)\right)\right)=0$ and $\varsigma^{*}\left(\widetilde{Z_{0}},\left\langle D_{1}, \ldots, D_{36}\right\rangle\right)=0$ (this follows from Proposition 2.7). By Lemma 8.13(iv), the element $\widetilde{Z_{0}}+D_{1}+\cdots+D_{36}$ is divisible by 2 . Hence, necessarily, there remain $7+\alpha$ elements $\frac{1}{2}\left(u_{1}+d_{1}\right), \ldots, \frac{1}{2}\left(u_{7+\alpha}+d_{7+\alpha}\right)$ in $H^{2}(\widetilde{K}, \mathbb{Z})$, linearly independent as elements of $H^{2}\left(\widetilde{K}, \mathbb{F}_{2}\right)$ and with $u_{i} \in \pi_{2 *}\left(s^{*}\left(H^{2}\left(K_{2}(A), \mathbb{Z}\right)\right)\right)$ and $d_{i} \in\left\langle D_{1}, \ldots, D_{36}\right\rangle$.

By Lemma $8.13($ iv $)$, the class $\left\langle D_{1}, \ldots, D_{36}\right\rangle$ is primitive in $H^{2}(\widetilde{K}, \mathbb{Z})$. Hence, necessarily, the elements $u_{1}, \ldots, u_{7+\alpha}$ viewed as elements in $\pi_{2 *}\left(s^{*}\left(H^{2}\left(K_{2}(A), \mathbb{F}_{2}\right)\right)\right)$ are also linearly independent. Since $\operatorname{dim}_{\mathbb{F}_{2}} \pi_{2 *}\left(s^{*}\left(H^{2}\left(K_{2}(A), \mathbb{F}_{2}\right)\right)\right)=7$, it follows that $\alpha=0$ and

$$
\operatorname{Vect}_{\mathbb{F}_{2}}\left(u_{1}, \ldots, u_{7}\right)=\pi_{2 *}\left(s^{*}\left(H^{2}\left(K_{2}(A), \mathbb{F}_{2}\right)\right)\right) \text {. }
$$

Hence, $e \in \operatorname{Vect}_{\mathbb{F}_{2}}\left(u_{1}, \ldots, u_{7}\right)$ and there exists a $D_{e}$ which is a linear combination of the $D_{i}$ with coefficients 0 or 1 such that $\pi_{2 *}\left(s^{*}(e)\right)+D_{e}$ is divisible by 2 .

Lemma 8.17. We have $D_{e}=D_{1}+\cdots+D_{36}$.

Proof. We know from Remark 6.5 that the image of the monodromy representation on $A[2]$ contains the symplectic group $\operatorname{Sp} A[2]$. We recall from Remark $7.7(2)$, that $D_{1}, \ldots, D_{35}$ are given by $\pi_{2}\left(s^{-1}(\mathcal{P})\right)$. It follows that the image of the monodromy representation on $H^{2}(\widetilde{K}, \mathbb{Z})$ contains the isometries which act on $D_{1}, \ldots, D_{35}$ as the elements $f$ of $\operatorname{Sp} A[2]$,

$$
f \cdot \pi_{2}\left(s^{-1}\left(\left\{a_{1}, a_{2}, a_{3}\right\}\right)\right)=\pi_{2}\left(s^{-1}\left(\left\{f\left(a_{1}\right), f\left(a_{2}\right), f\left(a_{3}\right)\right\}\right)\right),
$$

and act trivially on $D_{36}$ and $\pi_{2 *}\left(s^{*}(e)\right)$. As explained by Remark 6.9, Remark 7.7(2) and Proposition 6.7, the two orbits of the action of $\operatorname{Sp} A[2]$ on the set $\mathfrak{D}:=\left\{D_{1}, \ldots, D_{35}\right\}$ correspond to the two sets of isotropic and non-isotropic planes in $A[2]$. Hence, by Proposition 6.10(iii) and (iv), the action of $\operatorname{Sp} A[2]$ on the set $\mathfrak{D}$ has two orbits: one with 15 elements and another with 20 elements.

On the other hand, as we have mentioned, we can assume that $A=E_{\xi} \times E_{\xi}$, where $E_{\xi}$ is the elliptic curve introduced in Definition 8.1. Hence, there is the following automorphism group acting on $A$ :

$$
G_{\xi}:=\left\langle\left(\begin{array}{ll}
\xi & 0 \\
0 & 1
\end{array}\right),\left(\begin{array}{ll}
0 & 1 \\
1 & 0
\end{array}\right),\left(\begin{array}{ll}
1 & 1 \\
0 & 1
\end{array}\right)\right\rangle
$$




\section{S. Kapfer And G. Menet}

The group $G_{\xi}$ extends naturally to an automorphism group of $N_{2}$ (we recall that $N_{2}$ is defined in Section 8.1), which we also denote by $G_{\xi}$. Moreover, the action of $G_{\xi}$ restricts to the set $\mathfrak{D}$. Then by Lemma 8.2, the action of $G_{\xi}$ on $\mathfrak{D}$ has two orbits: one with 5 elements and one with 30 elements. Also, the group $G_{\xi}$ acts trivially on $D_{36}$ and $\pi_{2 *}\left(s^{*}(e)\right)$.

Hence, the combined action of $G_{\xi}$ and $\operatorname{Sp} A[2]$ is transitive on $\mathfrak{D}$. Since $\pi_{2 *}\left(s^{*}(e)\right)$ is fixed by the action of $G_{\xi}$ and $\operatorname{Sp} A[2]$, the divisor $D_{e}$ also has to be fixed by the action of $G_{\xi}$ and $\operatorname{Sp} A[2]$; else, we have a contradiction with Lemma 8.13(iv). It follows that there are only three possibilities for $D_{e}$ :

(1) $D_{e}=D_{36}$,

(2) $D_{e}=D_{1}+\cdots+D_{35}$, or

(3) $D_{e}=D_{1}+\cdots+D_{36}$.

Let $d$ be the number of $D_{i}$ with coefficient equal to 1 in the linear decomposition of $D_{e}$. The number $d$ can be 1,35 or 36 .

Then from Lemma 8.13(i) and (ii) and Proposition 2.7,

$$
\left(\frac{\pi_{2 *}\left(s^{*}(e)\right)+D_{e}}{2}\right)^{4}=\frac{324-d}{2} .
$$

Hence, $d$ has to be divisible by 2 . It follows that $D_{e}=D_{1}+\cdots+D_{36}$.

LEMma 8.18. The class $\pi_{1 *}\left(s_{1}^{*}(e)\right)+{\overline{Z_{0}}}^{\prime}$ is divisible by 2 .

Proof. We know that $\pi_{2, *}\left(s^{*}(e)\right)+\widetilde{\overline{Z_{0}}}$ is divisible by 2. Indeed, by Lemma 8.13(iv), the class $\widetilde{Z_{0}}+D_{1}+\cdots+D_{36}$ is divisible by 2 , and by Lemmas 8.16 and 8.17 , the class $\pi_{2, *}\left(s^{*}(e)\right)+D_{1}+$ $\cdots+D_{36}$ is divisible by 2 .

We can find a Cartier divisor on $\widetilde{K}$ which corresponds to $\frac{1}{2}\left(\pi_{2 *}\left(s^{*}(e)\right)+\widetilde{\overline{Z_{0}}}\right)$ and which does not meet $\cup_{k=1}^{36} D_{k}$. Then this Cartier divisor induces a Cartier divisor on $K^{\prime}$ which necessarily corresponds to half the cocycle $\pi_{1 *}\left(s_{1}^{*}(e)\right)+{\overline{Z_{0}}}^{\prime}$.

\subsection{Computation of $B_{K^{\prime}}$}

We finish the proof of Theorem 1.1, computing the Beauville-Bogomolov form $B_{K^{\prime}}$ of $K^{\prime}$. We continue using the notation provided in Section 8.1. One of the main ingredients will be the Fujiki formula (see [Men14, Section 1.2.2]). If $X=K^{\prime}$ or $K_{2}(A)$, we have, for all $\alpha \in H^{2}(X, \mathbb{Z})$,

$$
\alpha^{4}=c_{X} B_{X}(\alpha, \alpha)^{2},
$$

where $c_{X} \in \mathbb{Q}$ is the Fujiki constant. Moreover, if $0 \neq \omega$ is the holomorphic 2-from on $X$, we have

$$
B_{X}(\omega+\bar{\omega}, \omega+\bar{\omega})>0 .
$$

There also exists a polarized version of the Fujiki formula:

$$
\alpha_{1} \cdot \alpha_{2} \cdot \alpha_{3} \cdot \alpha_{4}=\frac{c_{X}}{24} \sum_{\sigma \in \mathfrak{S}_{4}} B_{X}\left(\alpha_{\sigma(1)}, \alpha_{\sigma(2)}\right) \cdot B_{X}\left(\alpha_{\sigma(3)}, \alpha_{\sigma(4)}\right)
$$

for all $\alpha_{i} \in H^{2}(X, \mathbb{Z})$.

LEMma 8.19. We have ${\overline{Z_{0}}}^{2}=-2 r^{*}\left(\overline{Z_{0}}\right)$. 


\section{INTEGRAL COHOMOLOGY OF THE GENERALIZED KUMMER FOURFOLD}

Proof. We use the same technique as in the proof of [Men14, Lemma 4.6.12]. Consider the following diagram:

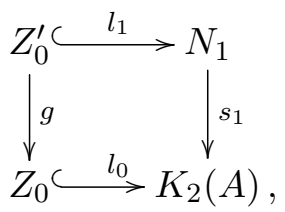

where $l_{0}$ and $l_{1}$ are the inclusions and $g:=s_{1 \mid Z_{0}^{\prime}}$. By [Ful98, Proposition 6.7], we have

$$
s_{1}^{*} l_{0 *}\left(Z_{0}\right)=l_{1 *}\left(c_{1}(E)\right),
$$

where $E:=g^{*}\left(\mathscr{N}_{Z_{0} / K_{2}(A)}\right) / \mathscr{N}_{Z_{0}^{\prime} / N_{1}}$. Hence,

$$
s_{1}^{*} l_{0 *}\left(Z_{0}\right)=c_{1}\left(g^{*}\left(\mathscr{N}_{Z_{0} / K_{2}(A)}\right)\right)-Z_{0}^{\prime 2} .
$$

Since $K_{2}(A)$ is hyper-Kähler and $Z_{0}$ is a K3 surface, we have $c_{1}\left(\mathscr{N}_{Z_{0} / K_{2}(A)}\right)=0$. So $Z_{0}^{\prime 2}=$ $-s_{1}^{*} l_{0 *}\left(Z_{0}\right)$. The result then follows from Proposition 2.7 .

Proposition 8.20. We have the formula

$$
B_{K^{\prime}}\left(\pi_{1 *}\left(s_{1}^{*}(\alpha), \pi_{1 *}\left(s_{1}^{*}(\beta)\right)\right)=6 \sqrt{\frac{2}{c_{K^{\prime}}}} B_{K_{2}(A)}(\alpha, \beta),\right.
$$

where $c_{K^{\prime}}$ is the Fujiki constant of $K^{\prime}$ and $\alpha, \beta$ are in $H^{2}\left(K_{2}(A), \mathbb{Z}\right)$ and $B_{K_{2}(A)}$ is the BeauvilleBogomolov form of $K_{2}(A)$.

Proof. The key ingredient for the proof is the Fujiki formula.

By (8.4), we have

$$
\begin{aligned}
\left(\pi_{1 *}\left(s_{1}^{*}(\alpha)\right)\right)^{4} & =c_{K^{\prime}} B_{K^{\prime}}\left(\pi_{1 *}\left(s_{1}^{*}(\alpha), \pi_{1 *}\left(s_{1}^{*}(\alpha)\right)\right)^{2},\right. \\
\alpha^{4} & =9 B_{K_{2}(A)}(\alpha, \alpha)^{2} .
\end{aligned}
$$

Moreover, by Proposition 2.7, we have $\left(\pi_{1 *}\left(s^{*}(\alpha)\right)\right)^{4}=8 s^{*}(\alpha)^{4}=8 \alpha^{4}$. The desired result follows from statement (8.5).

In particular, it follows that we have

$$
B_{K^{\prime}}\left(\pi_{1 *}\left(s_{1}^{*}(e), \pi_{1 *}\left(s_{1}^{*}(e)\right)\right)=-36 \sqrt{\frac{2}{c_{K^{\prime}}}} .\right.
$$

Lemma 8.21. The equality $B_{K^{\prime}}\left(\pi_{1 *}\left(s_{1}^{*}(\alpha)\right),{\overline{Z_{0}}}^{\prime}\right)=0$ holds for all $\alpha \in H^{2}\left(K_{2}(A), \mathbb{Z}\right)$.

Proof. We have $\pi_{1 *}\left(s_{1}^{*}(\alpha)\right)^{3} \cdot{\overline{Z_{0}}}^{\prime}=8 s_{1}^{*}(\alpha)^{3} \cdot \Sigma_{1}$ by Proposition 2.7 and $s_{1 *}\left(s_{1}^{*}\left(\alpha^{3}\right) \cdot Z_{0}^{\prime}\right)=\alpha^{3}$. $s_{1 *}\left(Z_{0}^{\prime}\right)=0$ by the projection formula. We conclude with (8.6).

Lemma 8.22. We have $B_{K^{\prime}}\left({\overline{Z_{0}}}^{\prime},{\overline{Z_{0}}}^{\prime}\right)=-4 \sqrt{2 / c_{K^{\prime}}}$.

Proof. By (8.6) and (8.7), we have

$$
\begin{aligned}
{\overline{Z_{0}}}^{\prime 2} \cdot \pi_{1 *}\left(s_{1}^{*}(e)\right)^{2} & =\frac{c_{K^{\prime}}}{3} B_{M^{\prime}}\left({\overline{Z_{0}}}^{\prime},{\overline{Z_{0}}}^{\prime}\right) \times B_{K^{\prime}}\left(\pi_{1 *}\left(s_{1}^{*}(e)\right), \pi_{1 *}\left(s_{1}^{*}(e)\right)\right) \\
& =\frac{c_{K^{\prime}}}{3} B_{K^{\prime}}\left({\overline{Z_{0}}}^{\prime},{\overline{Z_{0}}}^{\prime}\right) \times\left(-36 \sqrt{\frac{2}{c_{K^{\prime}}}}\right) \\
& =-12 \sqrt{2 c_{K^{\prime}}} B_{K^{\prime}}\left({\overline{Z_{0}}}^{\prime},{\overline{Z_{0}}}^{\prime}\right) .
\end{aligned}
$$


By Proposition 2.7, we have

$$
{\overline{Z_{0}}}^{\prime 2} \cdot \pi_{1 *}\left(s_{1}^{*}(e)\right)^{2}=8 Z_{0}^{\prime 2} \cdot\left(s_{1}^{*}(e)\right)^{2} .
$$

By the projection formula, $Z_{0}^{\prime 2} \cdot\left(s_{1}^{*}(e)\right)^{2}=s_{1 *}\left(Z_{0}^{\prime 2}\right) \cdot e^{2}$. Moreover, by Lemma 8.19 , we have $s_{1 *}\left(Z_{0}^{\prime 2}\right)=-Z_{0}$. Hence,

$$
Z_{0}^{\prime 2} \cdot\left(s_{1}^{*}(e)\right)^{2}=-Z_{0} \cdot e^{2}
$$

It follows from (8.8), (8.9) and (8.10) that

$$
-8 Z_{0} \cdot e^{2}=-12 \sqrt{2 c_{K^{\prime}}} B_{K^{\prime}}\left({\overline{Z_{0}}}^{\prime},{\overline{Z_{0}}}^{\prime}\right)
$$

Moreover, from [HT13, Section 4], we deduce

$$
Z_{0} \cdot e^{2}=-12
$$

So, by (8.11) and (8.12),

$$
B_{K^{\prime}}\left({\overline{Z_{0}}}^{\prime},{\overline{Z_{0}}}^{\prime}\right)=-8 \sqrt{\frac{1}{2 c_{K^{\prime}}}} .
$$

We are now able to finish the calculation of the Beauville-Bogomolov form on $H^{2}\left(K^{\prime}, \mathbb{Z}\right)$. By (8.7), Propositions 8.20, Lemmas 8.21 and 8.22 and Theorem 8.12, the Beauville-Bogomolov form on $H^{2}\left(K^{\prime}, \mathbb{Z}\right)$ gives the lattice

$$
U^{\oplus 3}\left(6 \sqrt{\frac{2}{c_{K^{\prime}}}}\right) \oplus-\frac{1}{4} \sqrt{\frac{2}{c_{K^{\prime}}}}\left(\begin{array}{cc}
40 & 32 \\
32 & 40
\end{array}\right)=U^{\oplus 3}\left(6 \sqrt{\frac{2}{c_{K^{\prime}}}}\right) \oplus-\sqrt{\frac{2}{c_{K^{\prime}}}}\left(\begin{array}{cc}
10 & 8 \\
8 & 10
\end{array}\right) .
$$

It then follows from the integrality and the indivisibility of the Beauville-Bogomolov form that $c_{K^{\prime}}=8$, and we get Theorem 1.1 .

\subsection{Betti numbers and Euler characteristic of $\boldsymbol{K}^{\prime}$}

Proposition 8.23. The Betti numbers and the Euler characteristic are $b_{2}\left(K^{\prime}\right)=8, b_{3}\left(K^{\prime}\right)=0$, $b_{4}\left(K^{\prime}\right)=90$ and $\chi\left(K^{\prime}\right)=108$.

Proof. The proof is the same as that of [Men14, Proposition 4.7.2]. We get the Betti numbers from [Voi02, Theorem 7.31], (7.2), (7.3) and Proposition 7.8. Then $\chi\left(K^{\prime}\right)=1-0+8-0+90-$ $0+8-0+1=108$.

\section{Appendix. Divisible classes in $H^{4}\left(K_{2}(A), \mathbb{Z}\right)$}

Here we give the divisible classes from Section 6.4 that were determined using a computer.

Proposition A.1. The following 31 classes of $\Pi^{\prime}$ are divisible by 3 in $H^{4}\left(K_{2}(A), \mathbb{Z}\right)$, and their thirds span an $\mathbb{F}_{3}$-vector space of dimension 31 in $\Pi^{\prime s a t} / \Pi^{\prime}$, namely $\sum_{\tau \in \Lambda}\left(Z_{\tau}-Z_{\tau+\tau^{\prime}}\right)$ :

$$
\begin{aligned}
\text { (i) } \quad & \Lambda=\left\langle\left(\begin{array}{l}
1 \\
0 \\
0 \\
0
\end{array}\right),\left(\begin{array}{l}
0 \\
1 \\
0 \\
0
\end{array}\right)\right\rangle \text { and } 0 \neq \tau^{\prime} \in P^{\perp}=\left\langle\left(\begin{array}{l}
0 \\
0 \\
1 \\
0
\end{array}\right),\left(\begin{array}{l}
0 \\
0 \\
0 \\
1
\end{array}\right)\right\rangle, \\
\text { (ii) } \quad & \Lambda=\left\langle\left(\begin{array}{l}
0 \\
0 \\
1 \\
0
\end{array}\right),\left(\begin{array}{l}
0 \\
0 \\
0 \\
1
\end{array}\right)\right\rangle \text { and } 0 \neq \tau^{\prime} \in P^{\perp}=\left\langle\left(\begin{array}{l}
1 \\
0 \\
0 \\
0
\end{array}\right),\left(\begin{array}{l}
0 \\
1 \\
0 \\
0
\end{array}\right)\right\rangle \backslash\left(\begin{array}{l}
1 \\
0 \\
0 \\
0
\end{array}\right), \\
\text { (iii) } \quad \Lambda & =\left\langle\left(\begin{array}{l}
1 \\
0 \\
0 \\
1
\end{array}\right),\left(\begin{array}{l}
0 \\
1 \\
2 \\
1
\end{array}\right)\right\rangle \text { and } \tau^{\prime} \in\left\{\left(\begin{array}{l}
0 \\
1 \\
1 \\
2
\end{array}\right),\left(\begin{array}{l}
1 \\
0 \\
0 \\
2
\end{array}\right),\left(\begin{array}{l}
1 \\
1 \\
1 \\
1
\end{array}\right),\left(\begin{array}{l}
2 \\
2 \\
2 \\
2
\end{array}\right)\right\} \\
\text { (iv) } \quad & \Lambda=\left\langle\left(\begin{array}{l}
1 \\
0 \\
0 \\
0
\end{array}\right),\left(\begin{array}{l}
0 \\
1 \\
0 \\
1
\end{array}\right)\right\rangle \text { and } \tau^{\prime} \in\left\{\left(\begin{array}{l}
0 \\
0 \\
0 \\
1
\end{array}\right),\left(\begin{array}{l}
1 \\
0 \\
1 \\
2
\end{array}\right),\left(\begin{array}{l}
1 \\
0 \\
2 \\
2 \\
2
\end{array}\right)\right\}
\end{aligned}
$$


INTEGRAL COHOMOLOGY OF THE GENERALIZED KUMMER FOURFOLD

$$
\begin{array}{ll}
\text { (v) } \quad \Lambda & =\left\langle\left(\begin{array}{l}
1 \\
0 \\
0 \\
0
\end{array}\right),\left(\begin{array}{l}
0 \\
1 \\
1 \\
1
\end{array}\right)\right\rangle \text { and } \tau^{\prime} \in\left\{\left(\begin{array}{l}
0 \\
0 \\
1 \\
1
\end{array}\right),\left(\begin{array}{l}
1 \\
0 \\
0 \\
1
\end{array}\right)\right\}, \\
\text { (vi) } \quad \Lambda & =\left\langle\left(\begin{array}{l}
1 \\
0 \\
1 \\
1
\end{array}\right),\left(\begin{array}{l}
0 \\
1 \\
0 \\
1
\end{array}\right)\right\rangle \text { and } \tau^{\prime} \in\left\{\left(\begin{array}{l}
0 \\
1 \\
0 \\
2
\end{array}\right),\left(\begin{array}{l}
1 \\
0 \\
2 \\
2
\end{array}\right)\right\}, \\
\text { (vii) } \quad \Lambda & =\left\langle\left(\begin{array}{l}
1 \\
0 \\
1 \\
0
\end{array}\right),\left(\begin{array}{l}
0 \\
1 \\
0 \\
1
\end{array}\right)\right\rangle \text { and } \tau^{\prime} \in\left\{\left(\begin{array}{l}
0 \\
1 \\
0 \\
2
\end{array}\right),\left(\begin{array}{l}
1 \\
0 \\
2 \\
0
\end{array}\right)\right\}, \\
\text { (viii) } \quad \Lambda & =\left\langle\left(\begin{array}{l}
1 \\
0 \\
0 \\
0
\end{array}\right),\left(\begin{array}{l}
0 \\
1 \\
0 \\
2
\end{array}\right)\right\rangle \text { and } \tau^{\prime}=\left(\begin{array}{l}
1 \\
0 \\
1 \\
0
\end{array}\right), \\
\text { (ix) } \quad \Lambda & =\left\langle\left(\begin{array}{l}
1 \\
0 \\
1 \\
1
\end{array}\right),\left(\begin{array}{l}
0 \\
1 \\
2 \\
2
\end{array}\right)\right\rangle \text { and } \tau^{\prime}=\left(\begin{array}{l}
1 \\
1 \\
0 \\
2
\end{array}\right) .
\end{array}
$$

Proposition A.2. We use Notation 5.13. The following 19 classes are divisible by 3 in $H^{4}\left(K_{2}(A), \mathbb{Z}\right)$, and their thirds provide a linear subspace of dimension 19 of the quotient $H^{4}\left(K_{2}(A), \mathbb{Z}\right) / \operatorname{Sym}^{\text {sat }} \oplus \Pi^{\prime \text { sat }}$ :

(i) $\quad u_{2}^{2}+\sum_{\tau \in \Lambda} Z_{\tau}-Z_{0}$ for $\Lambda=\left\langle\left(\begin{array}{l}0 \\ 0 \\ 0 \\ 1\end{array}\right),\left(\begin{array}{l}0 \\ 0 \\ 1 \\ 0\end{array}\right)\right\rangle$,

(ii) $\quad v_{2}^{2}+v_{2} u_{2}+u_{2}^{2}+\sum_{\tau \in \Lambda} Z_{\tau}-Z_{0} \quad$ for $\Lambda=\left\langle\left(\begin{array}{l}0 \\ 0 \\ 0 \\ 1\end{array}\right),\left(\begin{array}{l}0 \\ 1 \\ 1 \\ 0\end{array}\right)\right\rangle$,

(iii) $\quad w_{2}^{2}+w_{2} u_{2}+u_{2}^{2}+\sum_{\tau \in \Lambda} Z_{\tau}-Z_{0} \quad$ for $\quad \Lambda=\left\langle\left(\begin{array}{l}0 \\ 0 \\ 1 \\ 0\end{array}\right),\left(\begin{array}{l}0 \\ 1 \\ 0 \\ 1\end{array}\right)\right\rangle$,

(iv) $w_{2}^{2}-w_{2} u_{2}+u_{2}^{2}+\sum_{\tau \in \Lambda} Z_{\tau}-Z_{0} \quad$ for $\Lambda=\left\langle\left(\begin{array}{l}0 \\ 0 \\ 1 \\ 0\end{array}\right),\left(\begin{array}{l}0 \\ 1 \\ 0 \\ 2\end{array}\right)\right\rangle$,

(v) $\quad w_{2}^{2}-w_{2} v_{2}+w_{2} u_{2}+v_{2}^{2}+v_{2} u_{2}+u_{2}^{2}+\sum_{\tau \in \Lambda} Z_{\tau}-Z_{0} \quad$ for $\quad \Lambda=\left\langle\left(\begin{array}{l}0 \\ 0 \\ 1 \\ 2\end{array}\right),\left(\begin{array}{l}0 \\ 1 \\ 0 \\ 1\end{array}\right)\right\rangle$,

(vi) $\quad w_{1}^{2}+w_{1} u_{2}+u_{2}^{2}+\sum_{\tau \in \Lambda} Z_{\tau}-Z_{0} \quad$ for $\quad \Lambda=\left\langle\left(\begin{array}{l}0 \\ 0 \\ 0 \\ 1\end{array}\right),\left(\begin{array}{l}1 \\ 0 \\ 2 \\ 0\end{array}\right)\right\rangle$,

(vii) $\quad w_{1}^{2}-w_{1} u_{2}+u_{2}^{2}+\sum_{\tau \in \Lambda} Z_{\tau}-Z_{0} \quad$ for $\quad \Lambda=\left\langle\left(\begin{array}{l}0 \\ 0 \\ 0 \\ 1\end{array}\right),\left(\begin{array}{l}1 \\ 0 \\ 1 \\ 0\end{array}\right)\right\rangle$,

(viii) $\quad v_{1}^{2}+v_{1} u_{2}+u_{2}^{2}+\sum_{\tau \in \Lambda} Z_{\tau}-Z_{0} \quad$ for $\Lambda=\left\langle\left(\begin{array}{l}0 \\ 0 \\ 1 \\ 0\end{array}\right),\left(\begin{array}{l}1 \\ 0 \\ 0 \\ 1\end{array}\right)\right\rangle$,

(ix) $v_{1}^{2}-v_{1} u_{2}+u_{2}^{2}+\sum_{\tau \in \Lambda} Z_{\tau}-Z_{0} \quad$ for $\quad \Lambda=\left\langle\left(\begin{array}{l}0 \\ 0 \\ 1 \\ 0\end{array}\right),\left(\begin{array}{l}1 \\ 0 \\ 0 \\ 2\end{array}\right)\right\rangle$,

(x) $\quad v_{1}^{2}+v_{1} w_{1}-v_{1} u_{2}+w_{1}^{2}+w_{1} u_{2}+u_{2}^{2}+\sum_{\tau \in \Lambda} Z_{\tau}-Z_{0} \quad$ for $\quad \Lambda=\left\langle\left(\begin{array}{l}0 \\ 0 \\ 1 \\ 2\end{array}\right),\left(\begin{array}{l}1 \\ 0 \\ 0 \\ 2\end{array}\right)\right\rangle$,

(xi) $\quad v_{1}^{2}+v_{1} w_{1}-v_{1} w_{2}-v_{1} v_{2}+v_{1} u_{2}+w_{1}^{2}+w_{1} w_{2}+w_{1} v_{2}-w_{1} u_{2}+w_{2}^{2}-w_{2} v_{2}+w_{2} u_{2}$

$$
+v_{2}^{2}+v_{2} u_{2}+u_{2}^{2}+\sum_{\tau \in \Lambda} Z_{\tau}-Z_{0} \quad \text { for } \quad \Lambda=\left\langle\left(\begin{array}{l}
0 \\
0 \\
1 \\
2
\end{array}\right),\left(\begin{array}{l}
1 \\
1 \\
0 \\
1
\end{array}\right)\right\rangle,
$$

(xii) $\quad v_{1}^{2}-v_{1} w_{1}+v_{1} w_{2}-v_{1} v_{2}+v_{1} u_{2}+w_{1}^{2}+w_{1} w_{2}-w_{1} v_{2}+w_{1} u_{2}+w_{2}^{2}+w_{2} v_{2}-w_{2} u_{2}$ 


$$
\begin{aligned}
& +v_{2}^{2}+v_{2} u_{2}+u_{2}^{2}+\sum_{\tau \in \Lambda} Z_{\tau}-Z_{0} \quad \text { for } \quad \Lambda=\left\langle\left(\begin{array}{l}
0 \\
0 \\
1 \\
1
\end{array}\right),\left(\begin{array}{l}
1 \\
2 \\
0 \\
1
\end{array}\right)\right\rangle, \\
& \text { (xiii) } \quad u_{1}^{2}+\sum_{\tau \in \Lambda} Z_{\tau}-Z_{0} \quad \text { for } \quad \Lambda=\left\langle\left(\begin{array}{l}
0 \\
1 \\
0 \\
0
\end{array}\right),\left(\begin{array}{l}
1 \\
0 \\
0 \\
0
\end{array}\right)\right\rangle \\
& \text { (xiv) } \quad u_{1}^{2}-u_{1} v_{2}+v_{2}^{2}+\sum_{\tau \in \Lambda} Z_{\tau}-Z_{0} \quad \text { for } \quad \Lambda=\left\langle\left(\begin{array}{l}
0 \\
1 \\
0 \\
0
\end{array}\right),\left(\begin{array}{l}
1 \\
0 \\
0 \\
1
\end{array}\right)\right\rangle \\
& \text { (xv) } \quad u_{1}^{2}+u_{1} v_{2}+v_{2}^{2}+\sum_{\tau \in \Lambda} Z_{\tau}-Z_{0} \quad \text { for } \quad \Lambda=\left\langle\left(\begin{array}{l}
0 \\
1 \\
0 \\
0
\end{array}\right),\left(\begin{array}{l}
1 \\
0 \\
0 \\
2
\end{array}\right)\right\rangle \\
& \text { (xvi) } \quad u_{1}^{2}+u_{1} w_{1}+w_{1}^{2}+\sum_{\tau \in \Lambda} Z_{\tau}-Z_{0} \quad \text { for } \quad \Lambda=\left\langle\left(\begin{array}{l}
0 \\
1 \\
0 \\
2
\end{array}\right),\left(\begin{array}{l}
1 \\
0 \\
0 \\
0
\end{array}\right)\right\rangle \text {, } \\
& \text { (xvii) } \quad u_{1}^{2}+u_{1} w_{1}-u_{1} v_{2}+w_{1}^{2}+w_{1} v_{2}+v_{2}^{2}+\sum_{\tau \in \Lambda} Z_{\tau}-Z_{0} \quad \text { for } \quad \Lambda=\left\langle\left(\begin{array}{l}
0 \\
1 \\
0 \\
2
\end{array}\right),\left(\begin{array}{l}
1 \\
0 \\
0 \\
1
\end{array}\right)\right\rangle \text {, } \\
& \text { (xviii) } \quad u_{1}^{2}-u_{1} w_{1}+u_{1} w_{2}-u_{1} u_{2}+w_{1}^{2}+w_{1} w_{2}-w_{1} u_{2}+w_{2}^{2}+w_{2} u_{2}+u_{2}^{2}+\sum_{\tau \in \Lambda} Z_{\tau}-Z_{0} \\
& \text { for } \Lambda=\left\langle\left(\begin{array}{l}
0 \\
1 \\
0 \\
1
\end{array}\right),\left(\begin{array}{l}
1 \\
0 \\
1 \\
0
\end{array}\right)\right\rangle \\
& \text { (xix) } \quad u_{1}^{2}+u_{1} v_{1}-u_{1} w_{1}+v_{1}^{2}+v_{1} w_{1}+w_{1}^{2}+\sum_{\tau \in \Lambda} Z_{\tau}-Z_{0} \quad \text { for } \quad \Lambda=\left\langle\left(\begin{array}{l}
0 \\
1 \\
2 \\
1
\end{array}\right),\left(\begin{array}{l}
1 \\
0 \\
0 \\
0
\end{array}\right)\right\rangle \text {. }
\end{aligned}
$$

\section{ACKNOWLEDGements}

We want to thank Samuel Boissière, David Chataur, Brendan Hassett, Giovanni Mongardi, Marc Nieper-Wißkirchen and Ulrike Rieß for useful discussions. We also thank Samuel Boissière, Daniel Huybrechts and Marc Nieper-Wißkirchen for their hospitality.

\section{REFERENCES}

BCS16 S. Boissière, C. Camere and A. Sarti, Classification of automorphisms on a deformation family of hyper-Kähler four-folds by p-elementary lattices, Kyoto J. Math. 56 (2016), no. 3, 465-499; https://doi.org/10.1215/21562261-3600139.

Bea83 A. Beauville, Variétés Kähleriennes dont la première classe de Chern est nulle, J. Differential Geom. 18 (1983), no. 4, 755-782; https://doi.org/10.4310/jdg/1214438181.

BN07 S. Boissière and M. Nieper-Wißkirchen, Generating series in the cohomology of Hilbert schemes of points on surfaces, LMS J. Comput. Math. 10 (2007), 254-270; https://doi.org/10.1112/ S146115700000139X.

BNS11 S. Boissière, M. Nieper-Wißkirchen and A. Sarti, Higher dimensional Enriques varieties and automorphisms of generalized Kummer varieties, J. Math. Pures Appl. 95 (2011), no. 5, 553563; https://doi.org/10.1016/j.matpur.2010.12.003.

BNS13 _ Smith theory and irreducible holomorphic symplectic manifolds, J. Topol. 6 (2013), no. 2, 361-390; https://doi.org/10.1112/jtopol/jtt002.

Boi05 S. Boissière, Chern classes of the tangent bundle on the Hilbert scheme of points on the affine plane, J. Algebraic Geom. 14 (2005), no. 4, 761-787; https://doi.org/10.1090/ S1056-3911-05-00412-1. 


\section{INTEGRAL COHOMOLOGY OF THE GENERALIZED KUMMER FOURFOLD}

Bor86 C. Borcea, Diffeomorphisms of a K3 surface, Math. Ann. 275 (1986), no. 1, 1-4; https://doi. org/10.1007/BF01458579.

Bre72 G. E. Bredon, Introduction to compact transformation groups, Pure and Appl. Math., vol. 46 (Academic Press, New York - London, 1972).

Bri03 M. Britze, On the cohomology of generalized kummer varieties, Ph.D. Thesis, University of Cologne, Germany, 2003, available at http://www.math.uni-bonn.de/people/huybrech/ britze.pdf and at http://kups.ub.uni-koeln.de/1049/.

DM99 P. Deligne and J. W. Morgan, Notes on supersymmetry (following Joseph Bernstein), Quantum Fields and Strings: a Course for Mathematicians, Vols. 1, 2 (Princeton, NJ, 1996/1997) (Amer. Math. Soc., Providence, RI, 1999), 41-97.

Dol12 I. V. Dolgachev, Classical algebraic geometry. A modern view (Cambridge Univ. Press, Cambridge, 2012); https://doi.org/10.1017/CB09781139084437.

FM12 B. Farb and D. Margalit, A primer on mapping class groups, Princeton Math. Ser., vol. 49 (Princeton Univ. Press, Princeton, NJ, 2012).

Fog68 J. Fogarty, Algebraic families on an algebraic surface, Amer. J. Math 90 (1968), 511-521; https: //doi.org/10.2307/2373541.

Fuj81 A. Fujiki, A theorem on bimeromorphic maps of Kähler manifolds and its applications, Publ. Res. Inst. Math. Sci. 17 (1981), no. 2, 735-754; https://doi.org/10.2977/prims/ 1195185272.

Fuj83_ On primitively symplectic compact Kähler $V$-manifolds of dimension four, Classification of Algebraic and Analytic Manifolds (Katata, 1982), Progr. Math., vol. 39 (Birkhäuser Boston, Boston, MA, 1983), 71-250.

Fuj88_, Finite automorphism groups of complex tori of dimension two, Publ. Res. Inst. Math. Sci. 24 (1988), no. 1, 1-97; https://doi.org/10.2977/prims/1195175326.

Ful98 W. Fulton, Intersection theory, 2nd ed., Ergeb. Math. Grenzgeb. (3), vol. 2 (Springer-Verlag, Berlin, 1998); https://doi.org/10.1007/978-1-4612-1700-8.

Göt94 L. Göttsche, Hilbert schemes of zero-dimensional subschemes of smooth varieties, Lecture Notes in Math., vol. 1572 (Springer-Verlag, Berlin, 1994); https://doi.org/10.1007/BFb0073491.

GV94 E. Ghys and A. Verjovsky, Locally free holomorphic actions of the complex affine group, Geometric Study of Foliations (Tokyo, 1993) (World Sci. Publ., River Edge, NJ, 1994), 201-217.

HT13 B. Hassett and Y. Tschinkel, Hodge theory and Lagrangian planes on generalized Kummer fourfolds, Mosc. Math. J. 13 (2013), no. 1, 33-56; http://www.mathjournals.org/mmj/ 2013-013-001/2013-013-001-003.html.

Huy99 D. Huybrechts, Compact hyper-Kähler manifolds: basic results, Invent. Math. 135 (1999), no. 1, 63-113; https://doi.org/10.1007/s002220050280.

Huy12 , A global Torelli theorem for hyperkähler manifolds [after M. Verbitsky], Astérisque 348 (2012), Exp. No. 1040, x, 375-403; http://www.bourbaki.ens.fr/TEXTES/1040.pdf.

Kap17 S. Kapfer, Symmetric powers of symmetric bilinear forms, homogeneous orthogonal polynomials on the sphere and an application to compact Hyperkähler manifolds, Commun. Contemp. Math. 19 (2017), no. 2, 1650007; https://doi.org/10.1142/S0219199716500073.

Kir15 T. Kirschner, Period mappings with applications to symplectic complex spaces, Lecture Notes in Math., vol. 2140 (Springer, Cham, 2015); https://doi.org/10.1007/978-3-319-17521-8.

LQW02a W.-P. Li, Z. Qin and W. Wang, Hilbert schemes and $\mathcal{W}$ algebras, Int. Math. Res. Not. 2002 (2002), no. 27, 1427-1456; https://doi.org/10.1155/S1073792802110129.

LQW02b_ Vertex algebras and the cohomology ring structure of Hilbert schemes of points on surfaces, Math. Ann. 324 (2002), no. 1, 105-133; https://doi.org/10.1007/s002080200330.

LS03 M. Lehn and C. Sorger, The cup product of Hilbert schemes for K3 surfaces, Invent. Math. 152 (2003), no. 2, 305-329; https://doi.org/10.1007/s00222-002-0270-7. 


\section{S. Kapfer and G. Menet}

Mar07 E. Markman, Integral generators for the cohomology ring of moduli spaces of sheaves over Poisson surfaces, Adv. Math. 208 (2007), no. 2, 622-646; https://doi.org/10.1016/j.aim. 2006. 03.006 .

Mar10 - Integral constraints on the monodromy group of the hyperKähler resolution of a symmetric product of a K3 surface, Internat. J. Math. 21 (2010), no. 2, 169-223; https://doi. org/10.1142/S0129167X10005957.

Mar11_ A survey of Torelli and monodromy results for holomorphic-symplectic varieties, Complex and Differential Geometry, Springer Proc. Math., vol. 8 (Springer, Heidelberg, 2011), 257322; https://doi.org/10.1007/978-3-642-20300-8_15.

Mat15 D. Matsushita, On base manifolds of Lagrangian fibrations, Sci. China Math. 58 (2015), no. 3, 531-542; https://doi.org/10.1007/s11425-014-4927-7.

Men14 G. Menet, Cohomologie entière et fibration lagrangiennes sur certaines variétés holomorphiquement symplectiques singulières, Ph.D. Thesis, Lille 1 University, France, 2014, available at http://gregoire-menet.webnode.fr/ and at https://www.theses.fr/181720825.

Men15_, Beauville-Bogomolov lattice for a singular symplectic variety of dimension 4, J. Pure Appl. Algebra 219 (2015), no. 5, 1455-1495; https://doi.org/10.1016/j.jpaa.2014.06. 012.

MH73 J. Milnor and D. Husemoller, Symmetric bilinear forms, Ergeb. Math. Grenzgeb., vol. 73 (Springer-Verlag, New York - Heidelberg, 1973); https://doi.org/10.1007/ 978-3-642-88330-9.

Mil08 J.S. Milne, Abelian varieties, 2008, available at http://www.jmilne.org/math/.

MM17 E. Markman and S. Mehrtra, Hilbert schemes of K3 surfaces are dense in moduli, Math. Nacr. 290 (2017), no. 5-6, 876-884; https://doi.org/10.1002/mana.201600161.

Mon12 G. Mongardi, Symplectic involutions on deformations of K3 ${ }^{[2]}$, Cent. Eur. J. Math. 10 (2012), no. 4, 1472-1485; https://doi.org/10.2478/s11533-012-0073-z.

Mon13 O On natural deformations of symplectic automorphisms of manifolds of $K 3^{[n]}$ type, C. R. Math. Acad. Sci. Paris 351 (2013), no. 13-14, 561-564; https://doi.org/10.1016/ j.crma.2013.07.020.

Mon16 On the monodromy of irreducible symplectic manifolds, Algebr. Geom. 3 (2016), no. 3, 385-391; https://doi.org/10.14231/AG-2016-017.

MT07 D. Markushevich and A.S. Tikhomirov, New symplectic $V$-manifolds of dimension four via the relative compactified Prymian, Internat. J. Math. 18 (2007), no. 10, 1187-1224; https: //doi.org/10.1142/S0129167X07004503.

MTW15 G. Mongardi, K. Tari and M. Wandel, Prime order automorphisms of abelian surfaces: a latticetheoretic point of view, 2015, arXiv:1506.05679.

MTW18_, Automorphisms of generalised Kummer fourfolds, Manuscripta Math. 155 (2018), no. 3-4, 449-469; https://doi.org/10.1007/s00229-017-0942-7.

Mum70 D. Mumford, Abelian varieties, Tata Inst. Fund. Res. Stud. Math., vol. 5 (Tata Inst. Fund. Res., Bombay; Oxford University Press, London, 1970).

Nak97 H. Nakajima, Heisenberg algebra and Hilbert schemes of points on projective surfaces, Ann. of Math. 145 (1997), no. 2, 379-388; https://doi.org/10.2307/2951818.

Nam01 Y. Namikawa, Extension of 2-forms and symplectic varieties, J. reine angew. Math. 539 (2001), 123-147; https://doi.org/10.1515/crll.2001.070.

Nik80 V.V. Nikulin, Integral symmetric bilinear forms and some of their applications, Math. USSR Izv. 14 (1980), no. 1, 103-167; https://doi.org/10.1070/IM1980v014n01ABEH001060.

Ogu12 K. Oguiso, No cohomologically trivial non-trivial automorphism of generalized kummer manifolds, 2012, arXiv:1208.3750.

QW05 Z. Qin and W. Wang, Integral operators and integral cohomology classes of Hilbert schemes, Math. Ann. 331 (2005), no. 3, 669-692; https://doi.org/10.1007/s00208-004-0602-6. 


\section{INTEGRAL COHOMOLOGY OF THE GENERALIZED KUMMER FOURFOLD}

Rap08 A. Rapagnetta, On the Beauville form of the known irreducible symplectic varieties, Math. Ann. 340 (2008), no. 1, 77-95; https://doi.org/10.1007/s00208-007-0139-6.

Shi78 T. Shioda, The period map of Abelian surfaces, J. Fac. Sci. Univ. Tokyo Sect. IA Math. 25 (1978), no. 1, 47-59.

Spa56 E. Spanier, The homology of Kummer manifolds, Proc. Amer. Math. Soc. 7 (1956), no. 1, 155-160; https://doi.org/10.2307/2033261.

Tar15 K. Tari, Automorphismes des variétés de Kummer généralisées, Ph.D. Thesis, Poitiers University, France, 2015, available at http://theses.univ-poitiers.fr/notice/view/57658.

Tot16 B. Totaro, The integral cohomology of the Hilbert scheme of two points, Forum Math. Sigma 4 (2016), e8; https://doi.org/10.1017/fms.2016.5.

Voi02 C. Voisin, Hodge theory and complex algebraic geometry. I, Cambridge Stud. Adv. Math., vol. 76 (Cambridge Univ. Press, Cambridge, 2002); https://doi.org/10.1017/CB09780511615344.

Simon Kapfer simon.kapfer@math.uni-augsburg.de

Institute of Mathematics of Augsburg University, D-86159 Augsburg, Germany

Grégoire Menet gregoire.menet@u-bourgogne.fr

University of Campinas, Institudo de Matematica estatistica e Camputaçao Cientifica, Cx. Postal 6065 - CEP 13083-970-Campinas-SP, Brazil

Current address: University of Burgundy, IMB, 9 avenue Alain Savary 21000 Dijon, France 